\title{
The evolution of the large-scale structure of the universe: beyond the linear regime*
}

\author{
Francis Bernardeau \\ Institut de Physique Théorique, CEA, IPhT, F-91191 Gif-sur-Yvette, \\ CNRS, URA 2306, F-91191 Gif-sur-Yvette, France
}

\begin{abstract}
These lecture notes introduce analytical tools, methods and results describing the growth of cosmological structure beyond the linear regime. The presentation is focused on the single flow regime of the Vlasov-Poisson equation describing the development of gravitational instabilities in a pressureless fluid.

These notes include the introduction of diagrammatic representations of the standard perturbation theory with applications to the calculation of the so-called loop contributions to the power spectra. A large part of these notes is devoted to the exploration of the convergence properties of these terms from the contribution of both the long-wave modes and the short-wave modes. It is shown that the former can be addressed with the help of the eikonal approximation while the latter effect is partially screened out. The resulting performances of the two-loop corrections of the power spectra are then presented.

Finally other avenues that use different methods are explored. In particular it is shown how joint density and profile probability distribution functions can be constructed out of the multiple-variable cumulant generating functions computed at tree order.
\end{abstract}

\section{Contents}

1 Introduction

2 The single flow Vlasov-Poisson equation 4

2.1 The Vlasov equation . . . . . . . . . . . . . . . . . . . 4

2.2 Single flow approximation . . . . . . . . . . . . . .

2.3 The curl modes . . . . . . . . . . . . . . . . . . 7

3 The linear theory 7

3.1 The linear modes . . . . . . . . . . . . . . . . . . . . 7

3.2 The Green functions . . . . . . . . . . . . . . . . . . . . 8

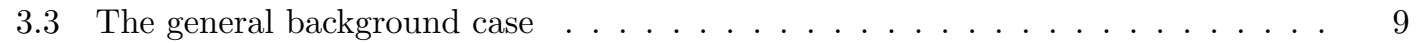

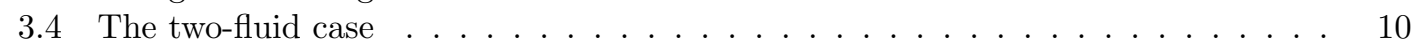

${ }^{*}$ Lectures given to the Les Houches Summer School Post-Planck Cosmology, 8 July - 2 August 2013 
4 Modes and statistics 12

4.1 The origin of stochasticity . . . . . . . . . . . . . . 12

4.2 Statistical homogeneity and isotropy . . . . . . . . . . . . . 14

4.3 Moments and cumulants . . . . . . . . . . . . . . . . [15

4.4 Moment and cumulant generating functions $\ldots \ldots \ldots \ldots \ldots \ldots$

5 The nonlinear equations

5.1 A field representation of the nonlinear motion equations . . . . . . . . . . 16

5.2 Diagrammatic representations . . . . . . . . . . . . . . . . 17

5.3 Scaling of solutions . . . . . . . . . . . . . . . . . . . . . [19

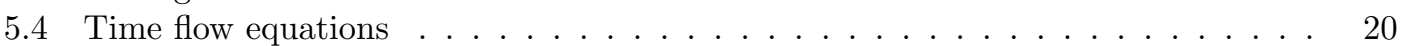

6 The infrared domain and the eikonal approximation 21

6.1 The IR behavior for the 1-loop corrections to the power spectrum . . . . . . . . . 21

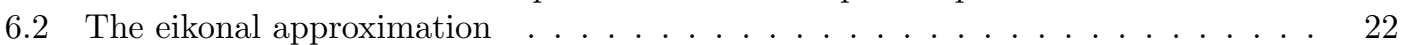

6.3 The extended Galilean invariance from the equivalence principle . . . . . . 24

7 The $\Gamma$ - expansion 25

7.1 The general formalism and theorem . . . . . . . . . . . . 25

7.2 The case of gravitational dynamics . . . . . . . . . . . . . 27

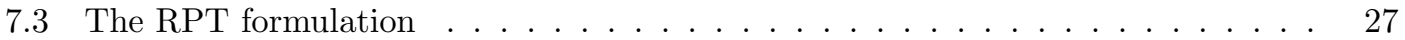

7.4 The MPTbreeze and RegPT formulation . . . . . . . . . . . . . 29

7.5 The performances of perturbation theory at NNLO . . . . . . . . . 29

8 Mode coupling structure 31

8.1 Scalings in the long wave mode regime . . . . . . . . . . . . 31

8.2 Kernels and integrands . . . . . . . . . . . . . . . . . . . 31

8.3 General convergence properties in the UV demain . . . . . . . . . . . . . . 32

9 Alternative perturbation theory schemes 34

9.1 Lagrangian PT . . . . . . . . . . . . . . . . . . . . . . . . . 34

9.2 The Zel'dovich approximation and higher order solutions . . . . . . . . . . 35

9.3 From displacement fields to power spectra . . . . . . . . . . . . . . 36

10 Other observables 37

10.1 The inverse Laplace transform . . . . . . . . . . . . . . . . . . 38

10.2 The Edgeworth expansion . . . . . . . . . . . . . . . . . . 39

11 Cumulants in spherical cells 40

11.1 Direct calculation of low order cumulants . . . . . . . . . . . . 40

11.2 The spherical collapse . . . . . . . . . . . . . . . . . 41

11.3 The tree order cumulant generating function as a Legendre transform of the initial

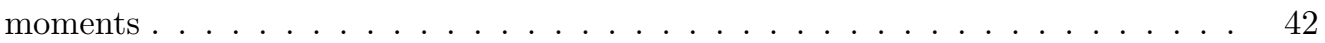

12 Density PDFs and profiles with spherical cells 44

12.1 Reconstructing the density PDFs . . . . . . . . . . . . 44

12.2 Two-cell cumulant generating functions $\ldots \ldots \ldots \ldots \ldots \ldots$

12.3 The expected density slope and profile . . . . . . . . . . . 47

13 Conclusion and perspectives 48 


\section{Introduction}

Observations made by the Planck mission and their interpretation represent undoubtedly the crown on our understanding of the development of gravitational instabilities throughout the cosmological ages (Planck Collaboration et al. 2013). It is nothing but the outcome of a scientific program that started about 30 years ago when the Cold Dark Matter (CDM) type cosmological models were first formulated (Peebles 1982, Blumenthal et al. 1984, Bond and Efstathiou 1984). The Planck observations that reach exquisite precision can indeed be explained with the help of only a few ingredients, an energy and matter content of the universe, a basic inflationary scenario. And within this scenario, the quality of robustness of the results is utterly impressive (see contribution of F.R. Bouchet, this volume, for more details). This is also the best that could be achieved out of the physics of linear perturbations in cosmology (see contribution of Matias Zaldarriaga, this volume), from the physics of the early universe, including an inflationary stage, the identification and evolution of the metric perturbations in general relativity to the development of perturbations are they re-enter within the Hubble radius including the physics of recombination captured by a Boltzmann equation.

The next frontier in our ability to confront observations and theory relies on the observational of large-scale structure of the universe in a regime which will necessarily incorporate non-linear effects, even though it could be at a mild level. This is the case in current and the next generation of surveys that should be able to exploit a large fraction of the observational universe, (see contribution of Will Percival, this volume). The analysis of these observations definitely requires tools and methods that go beyond the linear theory, and incidentally beyond the exploitation of statistical properties of a Gaussian or nearly Gaussian field.

The traditional approach to study the development of large-scale structure is to make use of numerical simulations (see contribution of Romain Teyssier). But although they provide very valuable insights into the late time development of gravitational instabilities they do not easily incorporate large dynamical range and are not necessary able to explore large variety of cosmological parameters or models. Obtaining results from first principles remains therefore extremely precious. The analytical investigations we will present here will be of interest for scales that range in between the linear physics and the fully nonlinear evolution, say between 100 and a few Mpc, and allows to apprehend the early departure from linear growth.

The purpose of these lectures is to provide specific tools and techniques and also to chart the state of the art for calculations from first principles. This is a domain that witnessed important development over the last few years with the introduction of novel methods. Standard perturbation theory (PT) calculations were described in Bernardeau et al. (2002) but since then a lot of efforts have been devoted to the development of alternative analytical methods that try to improve upon standard perturbation theory calculations. The first significant progress in this line of calculations is the Renormalized Perturbation Theory (RPT) proposition (Crocce and Scoccimarro 2006b) followed among other propositions by the closure theory (Taruya and Hiramatsu 2008) and the time flow equations approach (Pietroni 2008). Latest developments present results up to three-loop order (as in Bernardeau et al. 2012 | Blas et al. 2013a) with effective proposition such as MPTbreeze (Crocce et al. 2012a) and RegPT (Taruya et al. 2012) that incorporate 2-loop order calculations and are accompanied by publicly released codes. Concurrently, better understanding of the mathematical structure of the equation have been obtained, in particular the role of the extended galilean invariance (Blas et al. 2013b, Peloso and Pietroni 2013 Kehagias and Riotto 2013 Creminelli et al. 2013) or how to compute the impact of long wave modes through the eikonal approximations (Bernardeau et al. 2012). Finally, approaches that aim at circumventing the intrinsic limitation of first principle calculations, namely the single flow approximation, have been put forward. They are hybrid approaches, based on effective 
field modeling (Carrasco et al. 2012, Pietroni et al. 2012), that could fruitfully complement the standard approaches. The methods aforementioned aim at computing corrective terms to the basic observable such as power spectra. Many other avenues have been explored that aim at computing quantities that are complementary to poly-spectra observations and predictions. Some of these methods and techniques will also be described.

The plan of the lectures is the following. In Section 2, the single flow Vlasov-Poisson system is derived. In Section 3 the Green function of this system is presented, including the case of the multiple fluids and for an arbitrary background. With the help of Section 4 that presents the basic tools of statistical analysis of classical random fields, we can move into the description of the nonlinear system in Section 5 including its diagrammatic representation. The following sections, 6 to 8, present the structure of mode couplings for the one-loop and two-loop corrections to the power spectrum. These sections contain a presentation of the eikonal approximation and of the $\Gamma$-expansion theorem on which schemes such as the RPT and RegPT are based on and a presentation of the converging properties of the diagrams. The remaining sections of the notes present alternative approaches. In Section 9 alternative PT methods are presented with a particular emphasis on Lagrangian PT. In Section 10 examples of other observables are considered. Specific tools, based on the cumulant generating function, namely the derivation of the inverse Laplace transform and of the Edgeworth expansion, are given. Finally in Sections 11 and 12 we will see how one can take advantage of the spherical collapse solution to derive the cumulant generating function of the density in concentric cells. It is used to derive respectively the probability distribution functions of the density and its profile. Some perspectives are presented in Section 13 .

\section{The single flow Vlasov-Poisson equation}

We owe to G. Lemaître in papers dating back (1931) and (1934) the idea that the large-scale structure of the universe grew because of gravitational instabilities out of primordial small inhomogeneities. In the early thirties, however, basic knowledge on the thermal history of the universe were missing (not to mention the existence of dark matter) and this is only recently that this work program could be completed. The description of the growth of gravitational instabilities in the local universe is described in detail in classical textbooks (Peebles 1980) and more specifically in Bernardeau et al. (2002) regarding various basic aspects of perturbation theories.

The object of this section is to describe the growth of perturbation in the local universe where the Newton dynamical laws are assumed to apply. To be more precise it is assumed that the distances of interest are small compared to the curvature radius of the universe so that, as long as the local gravitational potentials are small, general relativity effects are effectively negligible.

\subsection{The Vlasov equation}

So let us start assuming that the universe is full of dust like particles whose only interaction is gravitational. For simplicity it is assumed that their masses are all the same, $m$.

The first step of the calculation is to introduce the phase space density function, $f(\mathbf{x}, \mathbf{p}) \mathrm{d}^{3} \mathbf{x ~ d}{ }^{3} \mathbf{p}$, which is the number of particles per volume element $\mathrm{d}^{3} \mathbf{x} \mathrm{d}^{3} \mathbf{p}$ where the position $\mathbf{x}$ of the particles is expressed in comoving coordinates and the particle conjugate momentum $\mathbf{p}$ reads

$$
\mathbf{p}=\mathbf{u} m a
$$

where $a$ is the expansion factor, $\mathbf{u}$ is the peculiar velocity, i.e. the difference of the physical velocity of the Hubble expansion. Then the conservation of the particles together with the 
Liouville theorem when the the two-body interactions can be neglected $\sqrt{1}$ implies that the total time derivative of $f$ vanishes so that

$$
\frac{\mathrm{d} f}{\mathrm{~d} t}=\frac{\partial}{\partial t} f(\mathbf{x}, \mathbf{p}, t)+\frac{\mathrm{d} \mathbf{x}}{\mathrm{d} t} \frac{\partial}{\partial \mathbf{x}} f(\mathbf{x}, \mathbf{p}, t)+\frac{\mathrm{d} \mathbf{p}}{\mathrm{d} t} \frac{\partial}{\partial \mathbf{p}} f(\mathbf{x}, \mathbf{p}, t)=0 .
$$

This is the Vlasov equation. The approach we are following here is actually very general and can be applied in a variety of contexts including the early stages of the dynamics. We restrict ourselves to the sub-Hubble scales that will make our derivation simple. The time variation of the position can be expressed in terms of $\mathbf{p}$ and one gets

$$
\frac{\mathrm{d} \mathbf{x}}{\mathrm{d} t}=\frac{\mathbf{p}}{m a^{2}} .
$$

The time variation of the momentum in general can be obtained from the geodesic equation. Assuming the metric perturbations are small and for scales much below the Hubble scale we have

$$
\frac{\mathrm{d} \mathbf{p}}{\mathrm{d} t}=-m \nabla_{\mathbf{x}} \Phi(\mathbf{x}, t)
$$

where $\Phi$ is the potential. We recall that in the context of metric perturbation in an expanding universe the potential $\Phi(\mathbf{x})$ is sourced by the density contrast (of all species). In our context we simply have

$$
\Delta \Phi(\mathbf{x})=\frac{4 \pi G m}{a}\left(\int f(\mathbf{x}, \mathbf{p}, t) \mathrm{d}^{3} \mathbf{p}-\bar{n}\right)
$$

where $\bar{n}$ is the spatial average of $\int f(\mathbf{x}, \mathbf{p}, t) \mathrm{d}^{3} \mathbf{p}$ and we then have

$$
\frac{\partial}{\partial t} f(\mathbf{x}, \mathbf{p}, t)+\frac{\mathbf{p}}{m a^{2}} \frac{\partial}{\partial \mathbf{x}} f(\mathbf{x}, \mathbf{p}, t)-m \nabla_{\mathbf{x}} \Phi(\mathbf{x}, t) \frac{\partial}{\partial \mathbf{p}} f(\mathbf{x}, \mathbf{p}, t)=0 .
$$

The system 6, 5) forms the Vlasov-Poisson equation. This is precisely the set of equations the $N$-body simulations attempt to solve.

We can now derive the basic conservation equations we are going to use from the first 2 moments of the Vlasov equation. Let us define the density field per volume $\mathrm{d}^{3} \mathbf{r}$ as

$$
\rho(\mathbf{x}, t)=\frac{m}{a^{3}} \int \mathrm{d}^{3} \mathbf{p} f(\mathbf{x}, \mathbf{p}) .
$$

It can be decomposed in an homogeneous form and an inhomogeneous form,

$$
\rho(\mathbf{x}, t)=\bar{\rho}(t)(1+\delta(\mathbf{x}, t))
$$

Note that $\bar{\rho}(t)$ the spatial averaged of $\rho(\mathbf{x}, t)$ should behave like $a(t)^{-3}$ for non relativistic species. One should then define the higher order moment of the phase space distribution: the mean velocity flow is defined as (for each component),

$$
u_{i}(\mathbf{x}, t)=\frac{1}{\int \mathrm{d}^{3} \mathbf{p} f(\mathbf{x}, \mathbf{p}, t)} \int \mathrm{d}^{3} \mathbf{p} \frac{p_{i}}{m a} f(\mathbf{x}, \mathbf{p}, t),
$$

and the second moment defines the velocity dispersion $\sigma_{i j}(\mathbf{x}, t)$,

$$
u_{i}(\mathbf{x}, t) u_{j}(\mathbf{x}, t)+\sigma_{i j}(\mathbf{x}, t)=\frac{1}{\int \mathrm{d}^{3} \mathbf{p} f(\mathbf{x}, \mathbf{p}, t)} \int \mathrm{d}^{3} \mathbf{p} \frac{p_{i}}{m a} \frac{p_{j}}{m a} f(\mathbf{x}, \mathbf{p}, t) .
$$

\footnotetext{
${ }^{1}$ Otherwise we would have to use the Boltzmann equation.
} 


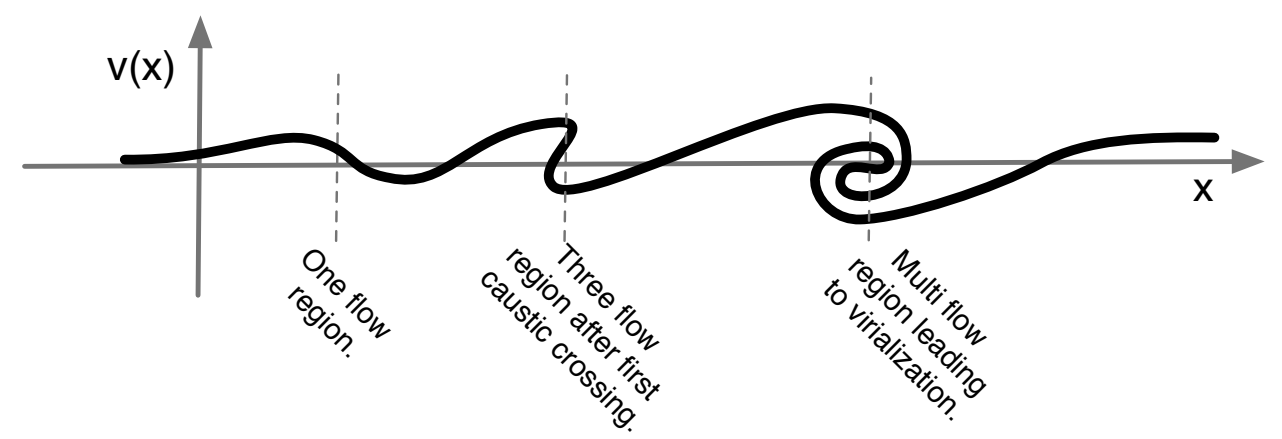

Figure 1: Schematic description of phase space after the first shell crossings and emergence of multi-flow regions. The figure is for $1 \mathrm{D}$ dynamics. From left to right, one can see regions with growing number of flows after dark matter caustic crossings.

The first two moments of the Vlasov equation give then the conservation and Euler equations, respectively

$$
\frac{\partial \delta(\mathbf{x}, t)}{\partial t}+\frac{1}{a}\left[(1+\delta(\mathbf{x}, t)) \mathbf{u}_{i}(\mathbf{x}, t)\right]_{, i}=0
$$

and

$$
\frac{\partial \mathbf{u}_{i}(\mathbf{x}, t)}{\partial t}+\frac{\dot{a}}{a} \mathbf{u}_{i}(\mathbf{x}, t)+\frac{1}{a} \mathbf{u}_{j}(\mathbf{x}, t) \mathbf{u}_{i}(\mathbf{x}, t)_{, j}=-\frac{1}{a} \Phi(\mathbf{x}, t)_{, i}-\frac{\left(\rho(\mathbf{x}, t) \sigma_{i j}(\mathbf{x}, t)\right)_{, j}}{\rho(\mathbf{x}, t) a} .
$$

The first term of the right hand side of eqn 12 is the gravitational force, the second is due to the pressure force which in general can be anisotropic. There are subsequent equations that can be written for the whole hierarchy of the velocity moments and depending of the physical situation, the hierarchy can be truncated if microphysics dictates a relation between the pressure tensor and the local density (this is the case for perfect fluid), if for some reasons the higher order moments become negligible as it is the case in the early phase of gravitational dynamics.

In the context we are interested in, the velocity actually vanishes until the formation of the first caustics.

\subsection{Single flow approximation}

The early stages of the gravitational instabilities are indeed characterized, assuming the matter is non-relativistic, by a negligible velocity dispersion when it is compared to the velocity flows, i.e. much smaller than the velocity gradients induced by the density fluctuations of the scales of interest. This is the single flow approximation. It simply states that one can assume

$$
f(\mathbf{x}, \mathbf{p}, t)=\frac{a^{3} \rho(\mathbf{x}, t)}{m} \delta^{(3)}[\mathbf{p}-m a \mathbf{u}(\mathbf{x}, t)],
$$

to a good approximation. This approximation will naturally break at the time of shell crossings when different flows - pulled toward one-another by gravity - cross. A sketch of what the phase space looks like is shown on Fig. 1. The multi-flow regions will eventually lead to the formation of astrophysical objects through a complicated phase of relaxation (virialization, see Binney and Tremaine (1987) for some hints on how that could take place). After shell crossings very little analytical results are known and one should rely on $N$-body codes. Within this approximation we simply have $\sigma_{i j}(\mathbf{x}, t)=0$. 
The Vlasov-Poisson equation in the single flow regime is the system that will be studied throughout these lecture notes, from linear to non nonlinear regime.

\subsection{The curl modes}

In the single flow regime, one can note that the source term of the Euler equation is potential, implying that it cannot generate any curl mode in the velocity field. More precisely, one can decompose any three-dimensional field in a gradient part and a curl part

$$
\mathbf{u}_{i}(\mathbf{x})=\psi(\mathbf{x})_{, i}+\mathbf{w}_{i}(\mathbf{x})
$$

where $\mathbf{w}_{i, i}=0$. Defining the local vorticity as

$$
\omega_{k}(\mathbf{x})=\epsilon^{i j k} \mathbf{u}_{i}(\mathbf{x})_{, j}
$$

where $\epsilon^{i j k}$ is the totally anti-symmetric Levi-Civita tensor one can easily show that

$$
\omega_{k}(\mathbf{x})=\epsilon^{i j k} \mathbf{w}_{i}(\mathbf{x})_{, j}
$$

and that applying the operator $\epsilon^{i j k} \nabla_{j}$ to the Euler equation one gets (see Bernardeau et al. 2002 )

$$
\frac{\partial}{\partial t} \omega_{k}+\frac{\dot{a}}{a} \omega_{k}-\epsilon^{i j k} \epsilon^{l m i}\left(\mathbf{u}_{l} \omega_{m}\right)_{, j}=0
$$

This equation actually expresses the fact that the vorticity is conserved throughout the expansion. In the linear regime that is when the last term of this equation is dropped it simply means that the vorticity scales like $1 / a$. In the subsequent stage of the dynamics the vorticity can only grow in contracting regions but it is still somehow conserved, it cannot be created out of potential modes only. That will be the case until shell crossing where the anisotropic velocity dispersion can then induce vorticity. This has been explicitly demonstrated in various studies (Pichon and Bernardeau 1999, Valageas 2007; Pueblas and Scoccimarro 2009). As a consequence, in the following, curl modes in the vector flied will always be neglected.

\section{The linear theory}

We now proceed to explore the linear regime of the Vlasov-Poisson system. One objective is to make contact with earlier stages of the gravitational dynamics and the second is to introduce the notion of Green function we will use in the following.

\subsection{The linear modes}

The linearization of the motion equation is obtained when one assumes that the terms $\left.[\delta(\mathbf{x}, t)) \mathbf{u}_{i}(\mathbf{x}, t)\right]_{, i}$ and $\mathbf{u}_{j}(\mathbf{x}, t) \mathbf{u}_{i}(\mathbf{x}, t)_{, j}$ in respectively the continuity and the Euler equation vanish. This is obtained when both the density contrast and the velocity gradients in units of $H$ are negligible. The linearized system is obtained in terms of the velocity divergence

$$
\theta(\mathbf{x}, t)=\frac{1}{a H} u_{i, i}
$$

so that the system now reads

$$
\begin{aligned}
\frac{\partial}{\partial t} \delta(\mathbf{x}, t)+H \theta(\mathbf{x}, t) & =0 \\
\frac{\partial}{\partial t} \theta(\mathbf{x}, t)+2 H \theta+\frac{\dot{H}}{H} \theta(\mathbf{x}, t) & =-\frac{3}{2} H \Omega_{m}(t) \delta(\mathbf{x}, t)
\end{aligned}
$$


after taking the divergence of the Euler equation. We have introduced here the Hubble parameter $H=\dot{a} / a$ and used the Friedman equation $H^{2}=8 \pi / 3 G \rho_{c}(t)$ together with the definition of $\Omega_{m}=\bar{\rho}(t) / \rho_{c}(t)$.

The resolution of this system is now simple. It can be obtained after eliminating the velocity divergence and one gets a second order dynamical equation,

$$
\frac{\partial^{2}}{\partial t^{2}} \delta(\mathbf{x}, t)+2 H \frac{\partial}{\partial t} \delta(\mathbf{x}, t)-\frac{3}{2} H^{2} \Omega_{m} \delta(\mathbf{x}, t)=0
$$

for the density contrast. It is to be noted that the spatial coordinates are here just labels: there is no operator acting of the physical coordinates. This is quite a unique feature in the growth of instabilities in a pressureless fluid. That implies in particular that the linear growth rate of the fluctuations will be independent on scale. The time dependence of the linear solution is given by the two solutions of

$$
\ddot{D}+2 H \dot{D}-\frac{3}{2} H^{2} \Omega_{m} D=0,
$$

one of which is decaying and the other is growing with time. For an Einstein de-Sitter (EdS) background (a universe with no curvature and with a critical matter density) the solutions read

$$
D_{+}^{\mathrm{EdS}}(t) \propto t^{2 / 3}, \quad D_{-}^{\mathrm{EdS}}(t) \propto 1 / t,
$$

that is $D_{+}^{\mathrm{EdS}}(t)$ is proportional to the expansion factor. This result gives the time scale of the growth of structure. This is what permits a direct comparison between the amplitude of the metric perturbations at recombination and the density perturbation in the local universe. Note that it implies that the potential, for the corresponding mode, is constant (see the Poisson equation).

In the following we will later see how these results can be extended to other background evolution.

\subsection{The Green functions}

The previous results show that the linear density field can be written in general

$$
\delta(\mathbf{x}, t)=\delta_{+}(\mathbf{x}) D_{+}(t)+\delta_{-}(\mathbf{x}) D_{-}(t)
$$

and

$$
\theta(\mathbf{x}, t)=-\frac{\mathrm{d}}{\mathrm{d} \log a} D_{+} \delta_{+}(\mathbf{x})-\frac{\mathrm{d}}{\mathrm{d} \log a} D_{-} \delta_{-}(\mathbf{x}) .
$$

The actual growing and decaying modes can then be obtained by inverting this system. For instance for an Einstein de Sitter background one gets

$$
\begin{aligned}
& \delta_{+}(\mathbf{x}) D_{+}(t)=\frac{D_{+}(t)}{D_{+}\left(t_{0}\right)}\left[\frac{3}{5} \delta\left(\mathbf{x}, t_{0}\right)-\frac{2}{5} \theta\left(\mathbf{x}, t_{0}\right)\right] \\
& \delta_{-}(\mathbf{x}) D_{-}(t)=\frac{D_{-}(t)}{D_{-}\left(t_{0}\right)}\left[\frac{2}{5} \delta\left(\mathbf{x}, t_{0}\right)+\frac{2}{5} \theta\left(\mathbf{x}, t_{0}\right)\right]
\end{aligned}
$$

and similar results for the velocity divergence. Following Scoccimarro (1998), this result can be encapsulated in a simple form after one introduces the doublet $\Psi_{a}(\mathbf{k}, \tau)$,

$$
\Psi_{a}(\mathbf{k}, \tau) \equiv(\delta(\mathbf{x}, t),-\theta(\mathbf{x}, t))
$$


where $a$ is an index whose value is either 1 (for the density component) or 2 (for the velocity component). The linear growth solution can now be written

$$
\Psi_{a}(\mathbf{x}, t)=g_{a}^{b}\left(t, t_{0}\right) \Psi_{b}\left(\mathbf{x}, t_{0}\right)
$$

where $g_{a}^{b}$ is the Green function of the system. It is usually written with the following time variable,

$$
\eta=\log D_{+}
$$

(not to be mistaken with the conformal time). For an Einstein de Sitter universe, we have explicitly,

$$
g_{a}^{b}\left(\eta, \eta_{0}\right)=\frac{e^{\eta-\eta_{0}}}{5}\left[\begin{array}{ll}
3 & 2 \\
3 & 2
\end{array}\right]+\frac{e^{-\frac{3}{2}\left(\eta-\eta_{0}\right)}}{5}\left[\begin{array}{cc}
2 & -2 \\
-3 & 3
\end{array}\right] .
$$

We will see in the following that, provided the doublet $\Psi_{a}$ is properly defined, this form remains practically unchanged for any background.

\subsection{The general background case}

For a general background, it is fruitful to extent the definition of the doublet to,

$$
\Psi_{a}(\mathbf{x}, \eta) \equiv\left(\delta(\mathbf{x}, \eta),-\frac{1}{f_{+}} \theta(\mathbf{x}, \eta)\right)
$$

where

$$
f_{+}=\frac{\mathrm{d} \log D_{+}}{\mathrm{d} \log a}
$$

Defining $\hat{\theta}=-\theta(\mathbf{x}, \eta) / f_{+}$and for the time variable $\eta$, the linearized motion equations indeed read

$$
\begin{aligned}
\frac{\partial}{\partial \eta} \delta(\mathbf{x}, \eta)-\hat{\theta}(\mathbf{x}, \eta) & =0 \\
\frac{\partial}{\partial \eta} \hat{\theta}(\mathbf{x}, \eta)\left(\frac{3}{2} \frac{\Omega_{m}}{f_{+}^{2}}-1\right) \hat{\theta}-\frac{3}{2} \frac{\Omega_{m}}{f_{+}^{2}} \delta(\mathbf{x}, \eta) & =0
\end{aligned}
$$

which can be rewritten as

$$
\frac{\partial}{\partial \eta} \Psi_{a}(\mathbf{x}, \eta)+\Omega_{a}^{b}(\eta) \Psi_{b}(\mathbf{x}, \eta)=0,
$$

with

$$
\Omega_{a}^{b}(\eta)=\left(\begin{array}{cc}
0 & -1 \\
-\frac{3}{2} \frac{\Omega_{m}}{f_{+}^{2}} & \frac{3}{2} \frac{\Omega_{m}}{f_{+}^{2}}-1
\end{array}\right) .
$$

In general, the formal solution of this system can be written in terms of a Green function $g_{a}^{b}\left(\eta, \eta_{0}\right)$. The latter satisfies the differential equation

$$
\frac{\partial}{\partial \eta} g_{a}^{b}\left(\eta, \eta_{0}\right)+\Omega_{a}^{c}(\eta) g_{c}^{b}\left(\eta, \eta_{0}\right)=0
$$

with the condition

$$
g_{a}^{b}\left(\eta_{0}, \eta_{0}\right)=\delta_{a}^{b},
$$




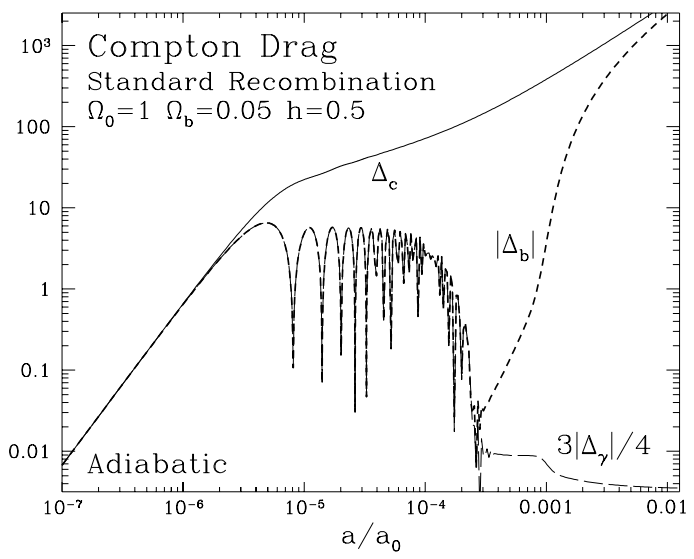

Figure 2: Cosmic evolution of the generalized density contrast $\Delta_{i}$ for radiation, $\Delta_{\gamma}$, dark matter, $\Delta_{c}$, and baryons, $\Delta_{b}$. One can see that instabilities start earlier for the dark matter component than for the baryon component. After decoupling the two-fluid follows the Vlasov-Poisson system and the linear solution is the superposition of the adiabatic growing mode and iso-density modes. From $\mathrm{Hu}(1995)$.

where $\delta_{a}{ }^{b}$ is the identity matrix. It is to be noted that the Green function can formally be written in terms of the peculiar solutions of the systems. For instance if one considers the growing and decaying solution $u_{a}^{(+)}(\eta)$ and $u_{a}^{(-)}(\eta)$, the Green function can be written

$$
g_{a}^{b}\left(\eta, \eta_{0}\right)=\sum_{\alpha=-,+} u_{a}^{(\alpha)}(\eta) c_{(\alpha)}^{b}\left(\eta_{0}\right)
$$

where the constants $c_{(\alpha)}^{b}\left(\eta_{0}\right)$ are set such that 39 is satisfied.

With the definition (32) of $\Psi_{a}$, the growing and decaying modes are, to a (surprisingly) good approximation, given by

$$
u_{a}^{(+)}=e^{\eta}\left(\begin{array}{c}
1 \\
1
\end{array}\right), \quad u_{a}^{(-)}=e^{-\frac{3}{2} \eta}\left(\begin{array}{c}
1 \\
-3 / 2
\end{array}\right) .
$$

This is due to the fact that $\Omega_{m} / f_{+}^{2} \approx 1$ in most regimes and models we consider.

As a result, in practice, we will always use the form (31) for the Green function.

\subsection{The two-fluid case}

In this paragraph I review the formalism for PT calculations in the presence of multiple pressureless fluids. The equations modeling multiple pressureless fluids and the resulting Green functions were first presented in Somogyi and Smith (2010).

We assume that the Universe is filled with pressureless fluids with only gravitational interactions. For $N$ fluids, we denote each fluid by a subscript $I(I=1, \ldots, N)$. Then, for each fluid the continuity equation reads

$$
\frac{\partial}{\partial t} \delta_{I}+\frac{1}{a}\left(\left(1+\delta_{I}\right) u_{I}^{i}\right)_{, i}=0
$$

while the Euler equation reads

$$
\frac{\partial}{\partial t} u_{I}^{i}+H u_{I}^{i}+\frac{1}{a} u_{I}^{j} u_{I, j}^{i}=-\frac{1}{a} \phi_{, i} .
$$


It is to be noted that the source term of the Poisson equation, $\Delta \phi=4 \pi G a^{2} \bar{\rho} \delta_{\mathrm{m}}$, is obviously the total mass density contrast defined by,

$$
\bar{\rho} \delta_{\mathrm{m}}=\sum_{I} \rho_{I} \delta_{I}
$$

Note that this system gives exactly the same motion equations for the total mass density contrast as the single flow case. It then admits the same linear solutions.

The linear behavior of the multi flow equations is conveniently described by the introduction of the multiplet $\Psi_{a}(a=1, \ldots, 2 N)$ (Scoccimarro 2001, Somogyi and Smith 2010, Bernardeau et al. 2012),

$$
\Psi_{a}=\left(\delta_{1}, \hat{\theta}_{1}, \delta_{2}, \hat{\theta}_{2}, \ldots\right)^{T}
$$

with $\hat{\theta}_{I} \equiv-\theta_{I} / f_{+}(t)$. The growth rate $f_{+}$is defined as the logarithmic change of the growth factor with the expansion, $f_{+} \equiv \mathrm{d} \ln D_{+} / \mathrm{d} a$, with $\mathrm{d} \eta \equiv \mathrm{d} \ln D_{+}$where $D_{+}$is the (unchanged) growing linear growth rate of the total density contrast. As before, the equations of motion can be recapped in the form,

$$
\frac{\partial}{\partial \eta} \Psi_{a}(\mathbf{x}, \eta)+\Omega_{a}^{b}(\eta) \Psi_{b}(\mathbf{x}, \eta)=0
$$

where the non-vanishing matrix elements of $\Omega_{a}^{b}$ are given by

$$
\Omega_{(2 I-1)}^{(2 I)}=-1, \quad \Omega_{(2 I)}^{(2 I)}=\frac{3}{2} \frac{\Omega_{\mathrm{m}}}{f_{+}^{2}}-1, \quad \Omega_{(2 I)}^{(2 J-1)}=-\frac{3}{2} \frac{\Omega_{\mathrm{m}}}{f_{+}^{2}} w_{q}
$$

and where we denote by $w_{I} \equiv \Omega_{I} / \Omega_{\mathrm{m}}$ the relative fraction of the fluid $I$.

This system has adiabatic solution, for which the fluids have all the same density contrast and velocity divergence, and iso-density modes. The latter are obtained under the constraint that the total density contrast vanishes, i.e.

$$
\delta_{\mathrm{m}}=\sum_{I} w_{I} \delta_{I}=0
$$

When we consider only 2 fluids, there are 2 such modes, one decaying and one constant in time. In the following we denote by "+" and "-" the growing and decaying adiabatic modes, respectively, and by " $\nabla$ " and " $<$ " the decaying and constant iso-density modes. Since under the constraint 48 the evolution equations decouple, the time dependence of these modes can be easily inferred. One solution is given by

$$
\begin{aligned}
\Theta_{I}^{(\nabla)}(\eta) & \propto \exp \left[-\int^{\eta} \mathrm{d} \eta^{\prime}\left(\frac{3}{2} \frac{\Omega_{\mathrm{m}}}{f_{+}^{2}}-1\right)\right], \\
\delta_{I}^{(\nabla)}(\eta) & =\int^{\eta} \mathrm{d} \eta^{\prime} \Theta_{I}^{(\nabla)}\left(\eta^{\prime}\right),
\end{aligned}
$$

with

$$
\sum_{I} w_{I} \Theta_{I}^{(\nabla)}=0
$$

which automatically ensures 48 . Note that, because $\Omega_{\mathrm{m}} / f_{+}^{2}$ departs only weakly from the value taken in an EdS cosmology, i.e. $\Omega_{\mathrm{m}} / f_{+}^{2}=1$, the iso-density modes are expected to depart very weakly from

$$
\Theta_{I}^{(\nabla)}(\eta) \propto \exp (-\eta / 2), \quad \delta_{I}^{(\nabla)}(\eta)=-2 \Theta_{I}^{(\nabla)}(\eta)
$$


A second set of isodensity modes is given by

$$
\Theta_{I}^{(<)}(\eta)=0, \quad \delta_{I}^{(<)}(\eta)=\text { Constant },
$$

under the condition that eqn 48 is satisfied.

To be specific, let us concentrate now on the case of two fluids and assume an EdS background. In this case, the growing and decaying solutions are then proportional, respectively, to

$$
\begin{aligned}
& u_{a}^{(+)}=(1,1,1,1)^{T}, \\
& u_{a}^{(-)}=(1,-3 / 2,1,-3 / 2)^{T} .
\end{aligned}
$$

Moreover, the isodensity modes are proportional to

$$
\begin{aligned}
& u_{a}^{(\nabla)}=\left(-2 w_{2}, w_{2}, 2 w_{1},-w_{1}\right)^{T} \\
& u_{a}^{(<)}=\left(w_{2}, 0,-w_{1}, 0\right)^{T} .
\end{aligned}
$$

If necessary we are then in the position to write down the linear propagator $g_{a}^{b}\left(\eta, \eta_{0}\right)$, from the general form of eqn 40 .

On Fig. 2 (taken from $\mathrm{Hu} 1995)$ one can see that just after recombination the two-fluid system, CDM and baryons, evolves in a way that involves both adiabatic and iso-density modes. The reason is that, at small scales, the baryons remain tightly coupled to the photons so that after recombination, their density contrasts and velocity modes are both damped.

\section{Modes and statistics}

\subsection{The origin of stochasticity}

In models of inflation the stochastic properties of the fields originate from quantum fluctuations of a scalar field, the inflaton. It is beyond the scope of this review to describe inflationary models in any detail. We instead refer the reader to standard reviews for a complete discussion (Linde 2005 Liddle and Lyth 2000, Lyth and Riotto 1999). It is worth however recalling that in such models (at least for the simplest single-field models within the slow-roll approximation) all fluctuations originate from scalar adiabatic perturbations. During the inflationary phase the energy density of the universe is dominated by the density stored in the inflaton field. This field has quantum fluctuations that can be decomposed in Fourier modes using the creation and annihilation operators $a_{\mathbf{k}}^{\dagger}$ and $a_{\mathbf{k}}$ for a wave mode $\mathbf{k}$,

$$
\delta \varphi=\int \mathrm{d}^{3} \mathbf{k}\left[a_{\mathbf{k}} \psi_{k}(t) \exp (\mathbf{i k} \cdot \mathbf{x})+a_{\mathbf{k}}^{\dagger} \psi_{k}^{*}(t) \exp (-\mathbf{i k} \cdot \mathbf{x})\right]
$$

The operators obey the standard commutation relation,

$$
\left[a_{\mathbf{k}}, a_{-\mathbf{k}^{\prime}}^{\dagger}\right]=\delta_{D}\left(\mathbf{k}+\mathbf{k}^{\prime}\right)
$$

and the mode functions $\psi_{k}(t)$ are obtained from the Klein-Gordon equation for $\varphi$ in an expanding Universe. We give here its expression for a de-Sitter metric (i.e. when the spatial sections are flat and $H$ is constant),

$$
\psi_{k}(t)=\frac{H}{(2 k)^{1 / 2} k}\left(\mathrm{i}+\frac{k}{a H}\right) \exp \left[\frac{\mathrm{i} k}{a H}\right],
$$




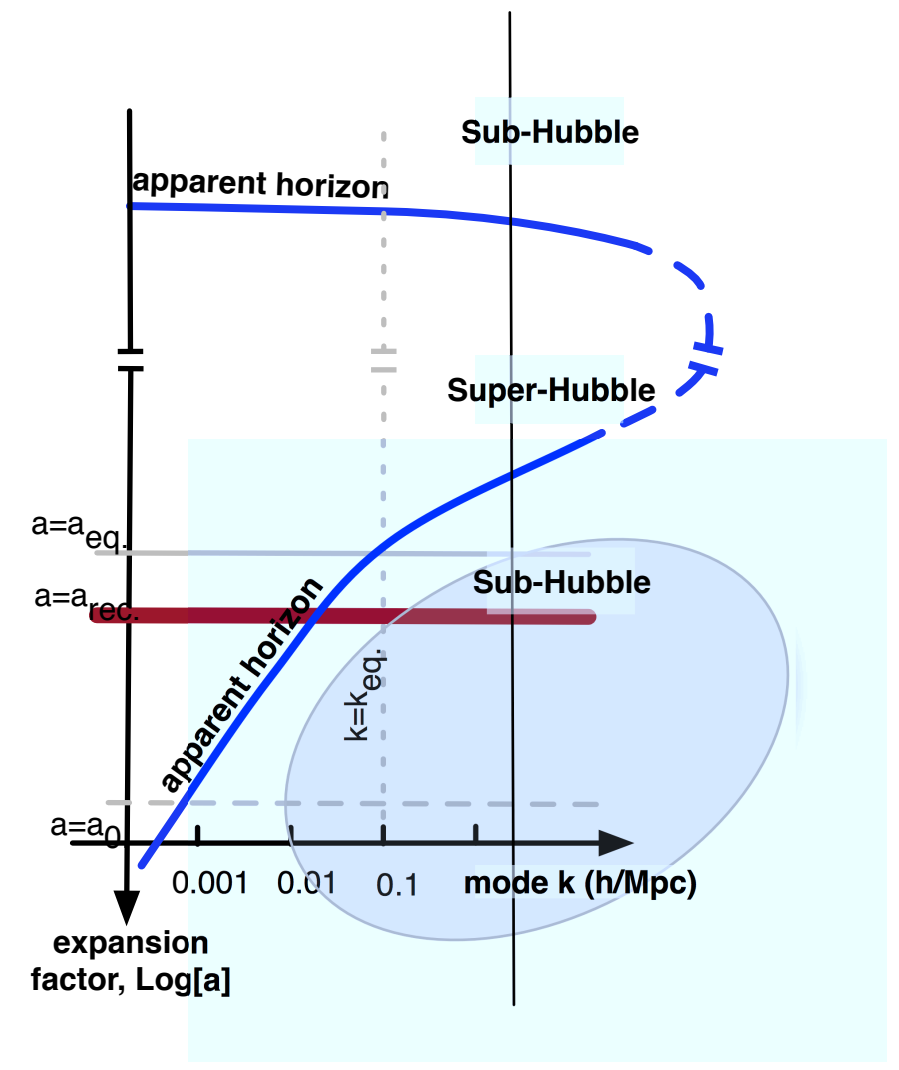

Figure 3: Development of linear metric perturbation across scale and time. This plot shows the different stages of perturbation growth as a function of the wave modes. For short wave-length (high k), (apparent) horizon crossing takes place before equality (time at which the radiation density and the matter density are equal). The growth of structure in the CDM component in then stalled from horizon crossing to equality, and resumes afterwards. Baryons participate in the growth of structure only after recombination. The motion equations we wrote are valid in the gray zone, at sub-Hubble scales and for pressureless fluids. In case of single field inflation, primordial fluid fluctuations are adiabatic: all fluid fluctuations that we see today then originate from a single scalar field degree of freedom. The nonlinear growth of perturbation will take place at low redshift and for large wave modes (bottom right of the figure). 
where $a$ and $H$ are respectively the expansion factor and the Hubble constant that are determined by the overall content of the Universe through the Friedmann equations.

When the modes exit the Hubble radius, $k /(a H) \ll 1$, one can see from eqn 60 that the dominant mode reads,

$$
\varphi_{\mathbf{k}} \approx \frac{i H}{\sqrt{2} k^{3 / 2}}\left(a_{\mathbf{k}}+a_{-\mathbf{k}}^{\dagger}\right), \quad \delta \varphi=\int \mathrm{d}^{3} \mathbf{k} \varphi_{\mathbf{k}} e^{\mathrm{i} \mathbf{k} \cdot \mathbf{x}}
$$

Therefore these modes are all proportional to $a_{\mathbf{k}}+a_{-\mathbf{k}}^{\dagger}$. One important consequence of this is that the quantum nature of the fluctuations has disappeared (Guth and Pi 1985; Kiefer et al. 1998): any combinations of $\varphi_{\mathbf{k}}$ commute with each other. The field $\varphi$ can then be seen as a classic stochastic field where ensemble averages identify with vacuum expectation values,

$$
\langle\ldots\rangle \equiv\langle 0|\ldots| 0\rangle \text {. }
$$

After the inflationary phase the modes re-enter the Hubble radius. They leave imprints of their energy fluctuations in the gravitational potential, the statistical properties of which can therefore be deduced from eqns (59,61). All subsequent stochasticity that appears in the cosmic fields can thus be expressed in terms of the random variable $\varphi_{\mathbf{k}}$. The linear theory calculation precisely tells us how each mode, in each fluid component, grows across time, i.e. it provides us with the so-called transfer functions, $T_{a}\left(\mathbf{k}, \eta, \eta_{0}\right)$, defined as

$$
\delta_{a}(\mathbf{k}, \eta)=T_{a}\left(\mathbf{k}, \eta, \eta_{0}\right) \delta \varphi\left(\mathbf{k}, \eta_{0}\right)
$$

where $\eta_{0}$ is a time which corresponds to an arbitrarily early time.

\subsection{Statistical homogeneity and isotropy}

In the following the density contrast will be decomposed in Fourier modes that, for a flat universe, are defined such as

$$
\delta(\mathbf{x})=\int \frac{\mathrm{d}^{3} \mathbf{k}}{(2 \pi)^{3 / 2}} \delta(\mathbf{k}) \exp (\mathrm{ik} \cdot \mathbf{x})
$$

or equivalently

$$
\delta(\mathbf{k})=\int \frac{\mathrm{d}^{3} \mathbf{x}}{(2 \pi)^{3 / 2}} \delta(\mathbf{x}) \exp (-\mathbf{i k} \cdot \mathbf{x})
$$

The observable quantities of interest are actually the statistical properties of such a field, whether it is represented in real space or in Fourier space. The Cosmological Principle, e.g. that the assumption that the Universe is statically isotropic and homogeneous, implies that real space correlators are homogeneous and isotropic which for instance implies that $\langle\delta(\mathbf{x}) \delta(\mathbf{x}+\mathbf{r})\rangle$ is a function of the separation $r$ only. This defines the two-point correlation function,

$$
\xi(r)=\langle\delta(\mathbf{x}) \delta(\mathbf{x}+\mathbf{r})\rangle
$$

In Fourier space, the two point correlator of the Fourier modes then takes the form,

$$
\begin{aligned}
\left\langle\delta(\mathbf{k}) \delta\left(\mathbf{k}^{\prime}\right)\right\rangle & =\int \frac{\mathrm{d}^{3} \mathbf{x}}{(2 \pi)^{3 / 2}} \frac{\mathrm{d}^{3} \mathbf{r}}{(2 \pi)^{3 / 2}} \xi(r) \exp \left[-\mathrm{i}\left(\mathbf{k}+\mathbf{k}^{\prime}\right) \cdot \mathbf{x}-\mathrm{ik}^{\prime} \cdot \mathbf{r}\right] \\
& =\delta_{\text {Dirac }}\left(\mathbf{k}+\mathbf{k}^{\prime}\right) \int \mathrm{d}^{3} \mathbf{r} \xi(r) \exp (\mathrm{ik} \cdot \mathbf{r}) \\
& \equiv \delta_{\text {Dirac }}\left(\mathbf{k}+\mathbf{k}^{\prime}\right) P(k)
\end{aligned}
$$




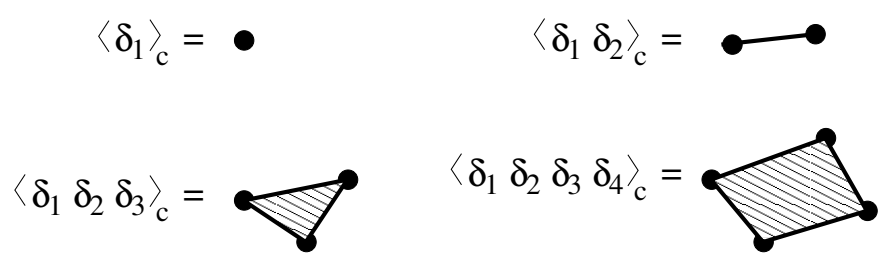

Figure 4: Representation of the connected part of the moments.

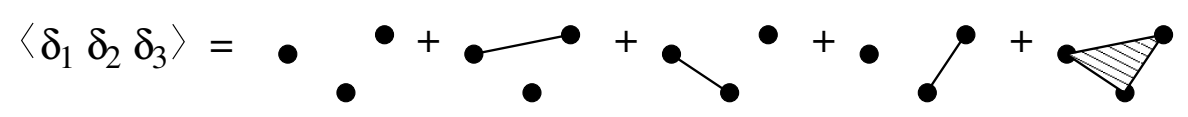

Figure 5: Writing of the three-point moment in terms of connected parts.

where $P(k)$ is the power spectrum of the density field, e.g. the cross-correlation matrix is symmetric in Fourier space.

All these relations apply to the observed fields. However in case of the primordial fluctuations, the field $\delta \varphi(\mathbf{k})$ corresponds to a free field from a quantum mechanical point of view. That makes it eventually a Gaussian classical field. As such it obeys the Wick theorem. The latter tells us that higher order correlators can then be entirely constructed from the power spectrum (from pair associations) through the relations,

$$
\begin{aligned}
\left\langle\delta \varphi\left(\mathbf{k}_{1}\right) \ldots \delta \varphi\left(\mathbf{k}_{2 p+1}\right)\right\rangle & =0 \\
\left\langle\delta \varphi\left(\mathbf{k}_{1}\right) \ldots \delta \varphi\left(\mathbf{k}_{2 p}\right)\right\rangle & =\sum_{\text {pair associations }} \prod_{p \text { pairs (i,j) }}\left\langle\delta \varphi\left(\mathbf{k}_{i}\right) \delta \varphi\left(\mathbf{k}_{j}\right)\right\rangle .
\end{aligned}
$$

These relations apply as well to any linear combinations of the primordial field, and therefore to any field computed in the linear regime.

\subsection{Moments and cumulants}

In the nonlinear regime however, fields also exhibit higher order non-trivial correlation functions that cannot be reconstructed from the two-point order correlators. They are defined as the connected part (denoted with subscript $c$ ) of the joint ensemble average of fields in an arbitrarily number of locations. Formally, for the density field, it reads,

$$
\begin{aligned}
\left\langle\delta\left(\mathbf{x}_{1}\right), \ldots, \delta\left(\mathbf{x}_{N}\right)\right\rangle_{c}= & \begin{array}{l}
\delta \\
\left.\left(\mathbf{x}_{1}\right), \ldots, \delta\left(\mathbf{x}_{N}\right)\right\rangle-
\end{array} \\
& -\sum_{\mathcal{S} \in \mathcal{P}\left(\left\{\mathbf{x}_{1}, \ldots, \mathbf{x}_{n}\right\}\right)} \prod_{s_{i} \in \mathcal{S}}\left\langle\delta\left(\mathbf{x}_{s_{i}(1)}\right), \ldots, \delta\left(\mathbf{x}_{s_{i}\left(\# s_{i}\right)}\right)\right\rangle,
\end{aligned}
$$

where the sum is made over the proper partitions (any partition except the set itself) of $\left\{\mathbf{x}_{1}, \ldots, \mathbf{x}_{N}\right\}$ and $s_{i}$ is thus a subset of $\left\{\mathbf{x}_{1}, \ldots, \mathbf{x}_{N}\right\}$ contained in partition $\mathcal{S}$. When the average of $\delta(\mathbf{x})$ is defined as zero, only partitions that contain no singlets contribute.

The decomposition in connected and non-connected parts can be easily visualized. It means that any ensemble average can be decomposed in a product of connected parts. They are defined for instance in Fig. 4. The tree-point moment is "written" in Fig. 5. Because of homogeneity of space $\left\langle\delta\left(\mathbf{k}_{1}\right) \ldots \delta\left(\mathbf{k}_{N}\right)\right\rangle_{c}$ is always proportional to $\delta_{D}\left(\mathbf{k}_{1}+\ldots+\mathbf{k}_{N}\right)$. Then we can define $P_{N}\left(\mathbf{k}_{1}, \ldots, \mathbf{k}_{N}\right)$ with

$$
\left\langle\delta\left(\mathbf{k}_{1}\right) \ldots \delta\left(\mathbf{k}_{N}\right)\right\rangle_{c}=\delta_{D}\left(\mathbf{k}_{1}+\ldots+\mathbf{k}_{N}\right) P_{N}\left(\mathbf{k}_{1}, \ldots, \mathbf{k}_{N}\right)
$$


One case of particular interest is for $n=3$, the bispectrum, which is usually denoted by $B\left(\mathbf{k}_{1}, \mathbf{k}_{2}, \mathbf{k}_{3}\right)$. Note that it depends on 2 wave modes only, and it depends on 3 independent variables characterizing the triangle formed by the 3 wave modes (for instance 2 lengths and 1 angle).

\subsection{Moment and cumulant generating functions}

It is convenient to define a function from which all moments can be generated, namely the moment generating function. It can be defined ${ }^{2}$ for any number of random variables. Here we give its definition for the local density field. It is defined by

$$
\mathcal{M}(t) \equiv \sum_{p=0}^{\infty} \frac{\left\langle\rho^{p}\right\rangle}{p !} t^{p}=\langle\exp (t \delta)\rangle
$$

The moments can obviously obtained by subsequent derivatives of this function at the origin $t=0$. A cumulant generating function can similarly be defined by

$$
\mathcal{C}(t) \equiv \sum_{p=2}^{\infty} \frac{\left\langle\rho^{p}\right\rangle_{c}}{p !} t^{p}
$$

A fundamental result is that the cumulant generating function is given by the logarithm of the moment generation function (see e.g. appendix D in Binney et al. (1992) for a proof)

$$
\mathcal{M}(t)=\exp [\mathcal{C}(t)]
$$

In case of a Gaussian probability distribution function, this is straightforward to check since $\langle\exp (t \delta)\rangle=\exp \left(\sigma^{2} t^{2} / 2\right)$.

\section{The nonlinear equations}

\subsection{A field representation of the nonlinear motion equations}

We now move to a full representation of the equations of motion, including the nonlinear terms that have been neglected so far. For that it is more convenient to go into the Fourier modes. More specifically we have,

$$
\begin{aligned}
{\left[\delta(\mathbf{x}) u_{i}(\mathbf{x})\right]_{, i} } & =\int \frac{\mathrm{d}^{3} \mathbf{k}_{1}}{(2 \pi)^{3 / 2}} \frac{\mathrm{d}^{3} \mathbf{k}_{2}}{(2 \pi)^{3 / 2}} \delta\left(\mathbf{k}_{1}\right) \theta\left(\mathbf{k}_{2}\right) \frac{\mathbf{k}_{2} \cdot\left(\mathbf{k}_{1}+\mathbf{k}_{2}\right)}{k_{2}^{2}} \exp \left(\mathrm{i}\left(\mathbf{k}_{1}+\mathbf{k}_{2}\right) \cdot \mathbf{x}\right) \\
{\left[u_{j}(\mathbf{x}) u_{i, j}(\mathbf{x})\right]_{, i} } & =\int \frac{\mathrm{d}^{3} \mathbf{k}_{1}}{(2 \pi)^{3 / 2}} \frac{\mathrm{d}^{3} \mathbf{k}_{2}}{(2 \pi)^{3 / 2}} \theta\left(\mathbf{k}_{1}\right) \theta\left(\mathbf{k}_{2}\right) \frac{\mathbf{k}_{1} \cdot \mathbf{k}_{2} \mathbf{k}_{1} \cdot\left(\mathbf{k}_{1}+\mathbf{k}_{2}\right)}{k_{1}^{2} k_{2}^{2}} \exp \left(\mathrm{i}\left(\mathbf{k}_{1}+\mathbf{k}_{2}\right) \cdot \mathbf{x}\right)(76)
\end{aligned}
$$

Plugging these expressions and taking the Fourier transform of these terms, one eventually gets, (Bernardeau et al. 2002)

$$
\frac{\partial}{\partial \eta} \Psi_{a}(\mathbf{k}, \eta)+\Omega_{a}^{b}(\eta) \Psi_{b}(\mathbf{k}, \eta)=\gamma_{a}^{b c}\left(\mathbf{k}_{1}, \mathbf{k}_{2}\right) \Psi_{b}\left(\mathbf{k}_{1}, \eta\right) \Psi_{c}\left(\mathbf{k}_{2}, \eta\right)
$$

\footnotetext{
${ }^{2}$ It is to be noted however that the existence of moments - which itself is not guaranteed for any stochastic process - does not ensure the existence of their generating function as the series defined in 72 can have a vanishing converging radius. Such a case is encountered for a lognormal distribution for instance and it implies that the moments of such a stochastic process do not uniquely define the probability distribution function, see Stoyanov (1987) for instance for details.
} 
where $\Omega_{a}^{b}(\eta)$ is defined in eqn (37) and where (and that will be the case henceforth) we use the convention that repeated Fourier arguments are integrated over and the Einstein convention on repeated indices, and where the symmetrized vertex matrix $\gamma_{a}^{b c}$ describes the non linear interactions between different Fourier modes. Its components are given by

$$
\begin{aligned}
& \gamma_{2}^{22}\left(\mathbf{k}_{1}, \mathbf{k}_{2}\right)=\delta_{\text {Dirac }}\left(\mathbf{k}-\mathbf{k}_{1}-\mathbf{k}_{2}\right) \frac{\left|\mathbf{k}_{1}+\mathbf{k}_{2}\right|^{2}\left(\mathbf{k}_{1} \cdot \mathbf{k}_{2}\right)}{2 k_{1}^{2} k_{2}^{2}}, \\
& \gamma_{1}{ }^{21}\left(\mathbf{k}_{1}, \mathbf{k}_{2}\right)=\delta_{\text {Dirac }}\left(\mathbf{k}-\mathbf{k}_{1}-\mathbf{k}_{2}\right) \frac{\left(\mathbf{k}_{1}+\mathbf{k}_{2}\right) \cdot \mathbf{k}_{1}}{2 k_{1}^{2}}
\end{aligned}
$$

$\gamma_{a}^{b c}\left(\mathbf{k}_{1}, \mathbf{k}_{2}\right)=\gamma_{a}^{c b}\left(\mathbf{k}_{2}, \mathbf{k}_{1}\right)$, and $\gamma=0$ otherwise, where $\delta_{\text {Dirac }}$ denotes the Dirac distribution function. The matrix $\gamma_{a}^{b c}$ is independent on time (and on the background evolution) and encodes all the non-linear couplings of the system.

One can then take advantage of the knowledge of the Green function of this system to write a formal solution of eqn (77) (Scoccimarro 1998; Scoccimarro 2001, Crocce and Scoccimarro $2006 \mathrm{~b}$, as

$$
\begin{aligned}
\Psi_{a}(\mathbf{k}, \eta) & =g_{a}^{b}(\eta) \Psi_{b}\left(\mathbf{k}, \eta_{0}\right)+ \\
& +\int_{\eta_{0}}^{\eta} \mathrm{d} \eta^{\prime} g_{a}^{b}\left(\eta, \eta^{\prime}\right) \gamma_{b}^{c d}\left(\mathbf{k}_{1}, \mathbf{k}_{2}\right) \Psi_{c}\left(\mathbf{k}_{1}, \eta^{\prime}\right) \Psi_{d}\left(\mathbf{k}_{2}, \eta^{\prime}\right),
\end{aligned}
$$

where $\Psi_{a}\left(\mathbf{k}, \eta_{0}\right)$ denotes the initial conditions.

In the following calculations we will be using the value of the $\Omega_{a}^{b}$ matrix to be that of the Einstein de Sitter background that is effectively assuming that $D_{-}$scales like $D_{+}^{-3 / 2}$. This is known to be a very good approximation even in the context of a $\Lambda-\mathrm{CDM}$ universe (see for instance Crocce et al. (2012b) for an explicit investigation of the consequences of this approximation).

\subsection{Diagrammatic representations}

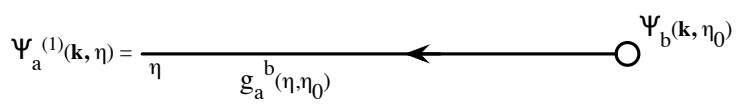

Figure 6: Diagrammatic representation of the linear propagator. $\Psi_{b}$ represents the initial conditions and $g_{a}^{b}$ is the time dependent propagator. This diagram value is the linear solution of the motion equation.

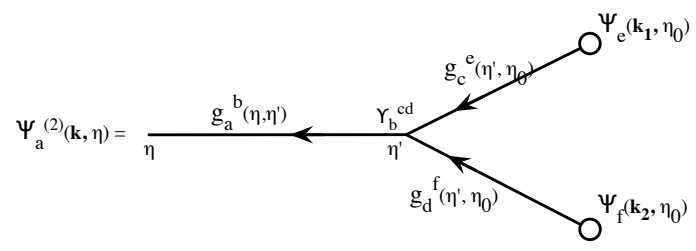

Figure 7: Diagrammatic representation of the fields at second order. This diagram value is given by eqn 79 when one replaces $\Psi_{c}$ and $\Psi_{d}$ in the second term of the right hand side by their linear expressions. In the diagram, each time one encounters a vertex, a time integration and a Dirac function in the wave modes is implicitly assumed. 


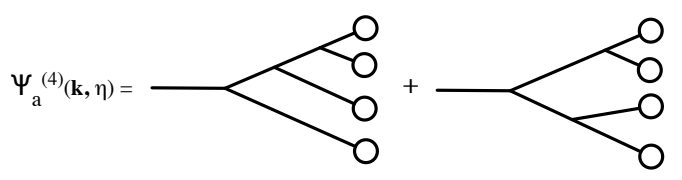

Figure 8: Diagrammatic representation of the fields at fourth order. Three different diagrams are found to contribute.

One of a nice feature of eqn $(79)$ is that it admits simple diagrammatic representations in a way very similar to Feynman diagrams.

A detailed description of the procedure to draw diagrams and compute their values can be found in Crocce and Scoccimarro (2006b), we can briefly summarize these rules here as follow ${ }^{3}$ In such representations, the open circles represent the initial conditions $\Psi_{b}\left(\mathbf{k}, \eta_{0}\right)$, where $b=1$ $(b=2)$ corresponds to the density (velocity divergence) field, and the line emerging from it carries a wavenumber $\mathbf{k}$. Lines are time-oriented (with time direction represented by an arrow) and have different indices at both ends, say $a$ and $b$. Each line represents linear evolution described by the propagator $g_{a}^{b}\left(\eta_{f}-\eta_{i}\right)$ from time $\eta_{i}$ to time $\eta_{f}$. The simplest diagram is presented on Fig. 6 It represents the linear growth solution.

Each nonlinear interaction between modes is represented by a vertex, which due to quadratic nonlinearities in the equations of motion is the convergence point of necessarily two incoming lines, with wavenumber say $\mathbf{k}_{1}$ and $\mathbf{k}_{2}$, and one outgoing line with wavenumber $\mathbf{k}=\mathbf{k}_{1}+\mathbf{k}_{2}$. Each vertex in a diagram then represents the matrix $\gamma_{a}^{b c}\left(\mathbf{k}_{1}, \mathbf{k}_{2}\right)$. It is further understood that internal indices are summed over and interaction times are integrated over the full interval $\left[0, \eta_{f}\right]$ as for instance in Fig. 7 where we present the diagrammatic expression of the second order expression of the fields. It is obtained after two modes computed at linear order, $\mathbf{k}_{1}$ and $\mathbf{k}_{2}$ interact together. Note that after the interaction the wave mode which is created is not necessarily in the growing modes. As a result, on this diagram both modes are propagating along the left line. This construction can obviously be extended to higher order in perturbation theory. On Fig. 8 the diagrams contributing to fourth order are shown.

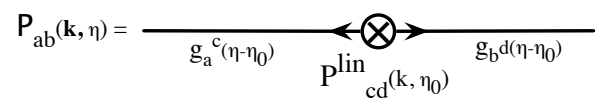

Figure 9: Diagrammatic representation of the power spectrum at linear order. The symbol $\otimes$ represents the linear power spectrum in the (adiabatic) growing mode.

Relevant statistical quantities are obtained however once ensemble average are taken. Assuming Gaussian initial conditions, one then can apply the Wick theorem to all the factors representing the initial field values that appear in diagrams (or product of diagrams) of interest. In practice, at least for these notes, the diagrams will all be computed assuming the initial conditions correspond effectively to the adiabatic linear growing mode. The simplest of such diagram is presented on Fig. 9. It corresponds to the ensemble average of $\left\langle\Psi_{a}(\mathbf{k}, \eta) \Psi_{b}(\mathbf{k}, \eta)\right\rangle$ and it makes intervene the linear power spectrum represented by $\otimes$. The previous construction can obviously be extended to any number of fields. The next diagrams will inevitably make intervene loops (in their diagram representation). One idea we will pursue here is to take advantage of such expansions to explore the density spectrum at 1-loop order and 2-loop order, also called at Next-to Leading Order and Next to Next to Leading Order (respectively NLO and NNLO).

\footnotetext{
${ }^{3}$ An alternative representation can be found in Matarrese and Pietroni (2007)
} 


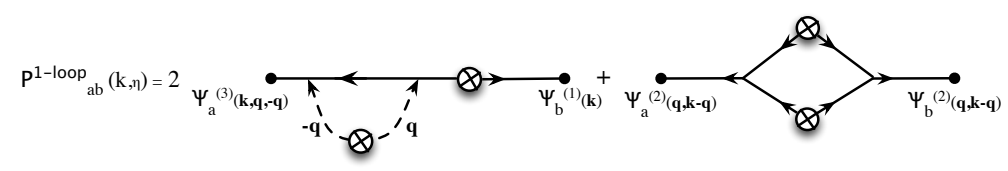

Figure 10: Diagrammatic representation of the 2 terms contributing to the power spectrum at one-loop order.

The 2 diagrams contributing to the power spectrum at NLO are shown on Fig. 10. The first calculation of such contributions were done in the 90's (Makino et al. 1992, Jain and Bertschinger 1996: Scoccimarro and Frieman 1996).

\subsection{Scaling of solutions}

It is interesting to compute the way subsequent orders in perturbation theory scale with the linear solution. As the vertices and the time integrations are both dimensionless operations, one can easily show that the $p$-th order expression of the density field is of the order of the power $p$ of the linear density field. In other words, there are kernels functions $F_{a}^{(n)}$ such that

$$
\begin{aligned}
\Psi_{a}^{(p)}(\mathbf{k}, \eta)= & \int \frac{\mathrm{d} \mathbf{k}_{1}}{(2 \pi)^{3 / 2}} \cdots \frac{\mathrm{d} \mathbf{k}_{p}}{(2 \pi)^{3 / 2}} \delta_{\text {Dirac }}\left(\mathbf{k}-\mathbf{k}_{1 \ldots p}\right) \\
& \times \mathcal{F}_{a}^{(p)}\left(\mathbf{k}_{1}, \ldots, \mathbf{k}_{p} ; \eta\right) \delta_{+}\left(\mathbf{k}_{1}, \eta\right) \ldots \delta_{+}\left(\mathbf{k}_{p}, \eta\right)
\end{aligned}
$$

where $\delta_{+}\left(\mathbf{k}_{i}, \eta\right)$ is the linear growing mode for wave modes $\mathbf{k}_{i}, \mathbf{k}_{1 \ldots p}=\mathbf{k}_{1}+\ldots+\mathbf{k}_{p}$ and where $\mathcal{F}_{a}^{(p)}\left(\mathbf{k}_{1}, \ldots, \mathbf{k}_{p} ; \eta\right)$ are dimensionless functions of the wave modes and are a priori time dependent. For an Einstein-de Sitter background, the functions $\mathcal{F}_{a}^{(p)}\left(\mathbf{k}_{1}, \ldots, \mathbf{k}_{p} ; \eta\right)$ are actually time independent and in general depends only very weakly on time (and henceforth on the cosmological parameters.) The functions $\mathcal{F}_{a}^{(p)}$ are usually noted $F_{p}$ and $G_{p}$ for respectively $a=1$ and $a=2$. For instance it is easy to show that

$$
F_{2}\left(\mathbf{k}_{1}, \mathbf{k}_{2}\right)=\frac{5}{7}+\frac{1}{2} \frac{\mathbf{k}_{1} \cdot \mathbf{k}_{2}}{k_{1}^{2}}+\frac{1}{2} \frac{\mathbf{k}_{1} \cdot \mathbf{k}_{2}}{k_{2}^{2}}+\frac{2}{7} \frac{\left(\mathbf{k}_{1} \cdot \mathbf{k}_{2}\right)^{2}}{k_{1}^{2} k_{2}^{2}}
$$

for an Einstein-de Sitter universe. For an arbitrary background the coefficient 5/7 and 2/7 are slightly altered but only very weakly (Bouchet et al. 1992).

It can be noted that this kernel is very general and is actually directly observable. Indeed for Gaussian initial conditions the first non-vanishing contribution to the bi-spectrum is obtained when one, and only one, factor is written at second order in the initial field,

$$
\left\langle\delta\left(\mathbf{k}_{1}\right) \delta\left(\mathbf{k}_{2}\right) \delta\left(\mathbf{k}_{3}\right)\right\rangle_{c}=\left\langle\delta^{(1)}\left(\mathbf{k}_{1}\right) \delta^{(1)}\left(\mathbf{k}_{2}\right) \delta^{(2)}\left(\mathbf{k}_{3}\right)\right\rangle_{c}+\text { sym. }
$$

and it is easy to show that it eventually reads

$$
\left\langle\delta\left(\mathbf{k}_{1}\right) \delta\left(\mathbf{k}_{2}\right) \delta\left(\mathbf{k}_{3}\right)\right\rangle_{c}=\delta_{\text {Dirac }}\left(\mathbf{k}_{1}+\mathbf{k}_{2}+\mathbf{k}_{3}\right)\left[2 F_{2}\left(\mathbf{k}_{1}, \mathbf{k}_{2}\right) P^{\text {lin. }}\left(k_{1}\right) P^{\text {lin. }}\left(k_{2}\right)+\text { sym. }\right]
$$

where sym. refers to 2 extra terms obtained by circular changes of the indices. The important consequence of this form is that the bispectrum therefore scales like the square of the power spectrum. In particular the reduced bispectrum defined as

$$
Q\left(\mathbf{k}_{1}, \mathbf{k}_{2}, \mathbf{k}_{3}\right)=\frac{B\left(\mathbf{k}_{1}, \mathbf{k}_{2}, \mathbf{k}_{3}\right)}{P\left(k_{1}\right) P\left(k_{2}\right)+P\left(k_{2}\right) P\left(k_{3}\right)+P\left(k_{3}\right) P\left(k_{1}\right)}
$$


is expected to have a time independent amplitude at early time.

More generally, the connected p-point correlators at lowest order in perturbation theory scale like the power $p-1$ of the 2-point correlators (Fry 1984, Goroff et al. 1986): it comes from the fact that in order to connect $p$ points using the Wick theorem one needs at least $p-1$ lines connecting a product of $2(p-1)$ fields taken at linear order. For instance the local $p$-order cumulant of the local density contrast $\left\langle\delta^{p}\right\rangle_{c}$ scales like,

$$
\left\langle\delta^{p}\right\rangle_{c} \sim\left\langle\delta^{2}\right\rangle^{p-1} .
$$

In the last sections of these notes, more detailed presentation of these relations will be given.

Other consequences of these scaling results concern the p-loop corrections to the power spectrum. Indeed one expects to have

$$
P^{\text {p-loop }}(k) \sim P^{\text {lin. }}(k)\left[\int \frac{\mathrm{d} q}{q} q^{3} P(q)\right]^{p} .
$$

At least that would be the case if the linear power spectrum peaked at wave modes about $k$. This is not necessarily the case. In the following we explore the mode coupling structure, i.e. how modes $q$ are contributing to the corrective terms to the power spectrum depending on whether they are much smaller (infrared domain) or much larger (ultra-violet domain) than $k$.

\subsection{Time flow equations}

Although in this presentation we will focus on the diagrammatic representation of the motion equations, and their integral form, there exists an alternative set of differential equations that gives the time dependence of the multi-point spectra. These equations form a hierarchy and we will give here the first two. The evolution equation 777 indeed allows to compute the time derivative of products such as $\Psi_{a}(\mathbf{k}, \eta) \Psi_{b}\left(\mathbf{k}^{\prime}, \eta\right)$ or $\Psi_{a}\left(\mathbf{k}_{1}, \eta\right) \Psi_{b}\left(\mathbf{k}_{2}, \eta\right) \Psi_{c}\left(\mathbf{k}_{3}, \eta\right)$. After taking their ensemble averages, it leads respectively to the following equations (see Pietroni 2008),

$$
\begin{aligned}
& \frac{\partial}{\partial \eta} P_{a b}(\mathbf{k}, \eta)=-\Omega_{a}^{c}(\mathbf{k}, \eta) P_{c b}(\mathbf{k}, \eta)-\Omega_{b}^{c}(\mathbf{k}, \eta) P_{a c}(\mathbf{k}, \eta) \\
& +\int d^{3} q\left[\gamma_{a}^{c d}(-\mathbf{q}, \mathbf{q}-\mathbf{k}) B_{b c d}(\mathbf{k},-\mathbf{q}, \mathbf{q}-\mathbf{k} ; \eta)+\gamma_{b}^{c d}(-\mathbf{q}, \mathbf{q}-\mathbf{k}) B_{a c d}(\mathbf{k},-\mathbf{q}, \mathbf{q}-\mathbf{k} ; \eta)\right]
\end{aligned}
$$

and

$$
\begin{aligned}
& \frac{\partial}{\partial \eta} B_{a b c}(\mathbf{k},-\mathbf{q}, \mathbf{q}-\mathbf{k} ; \eta)=-\Omega_{a}^{d}(\mathbf{k}, \eta) B_{d b c}(\mathbf{k},-\mathbf{q}, \mathbf{q}-\mathbf{k} ; \eta) \\
& -\Omega_{b}^{d}(-\mathbf{q}, \eta) B_{a d c}(\mathbf{k},-\mathbf{q}, \mathbf{q}-\mathbf{k} ; \eta)-\Omega_{c}^{d}(\mathbf{q}-\mathbf{k}, \eta) B_{a b d}(\mathbf{k},-\mathbf{q}, \mathbf{q}-\mathbf{k} ; \eta) \\
& +2\left[\gamma_{a}^{d e}(-\mathbf{q}, \mathbf{q}-\mathbf{k}) P_{d b}(\mathbf{q}, \eta) P_{e c}(\mathbf{k}-\mathbf{q}, \eta)+\gamma_{b}^{d e}(\mathbf{q}-\mathbf{k}, \mathbf{k}) P_{d c}(\mathbf{k}-\mathbf{q}, \eta) P_{e a}(\mathbf{k}, \eta)\right. \\
& \left.\quad+\gamma_{c}^{d e}(\mathbf{k},-\mathbf{q}) P_{d a}(\mathbf{k}, \eta) P_{e b}(\mathbf{q}, \eta)\right],
\end{aligned}
$$

where in the latter equation the connected parts of the four-point correlators have been dropped. It can be easily checked that taking the power spectrum at linear order in the right hand side of eqn (88) gives back the standard perturbation theory results at one-loop order. Those results can then be used as an alternative scheme to obtain Perturbation Theory results without relying neither on the explicit form of the Green function nor on diagrammatic expansions. It is then of interest for systems that are richer than pure dark matter systems (for instance with massive neutrinos as in Lesgourgues et al. 2009). 
This approach has been advocated though as an alternative approach to standard perturbation theory. Indeed in eqn (88) if one uses the non-linear power spectrum then eqns (87) and (88) form a closed system of equation which provides a NLO calculation of the power spectrum which is distinct from standard Perturbation Theory result. In principle such expansions can be pursued to higher order introducing a system which involves also the tri-spectrum, etc.

\section{The infrared domain and the eikonal approximation}

On of the reason for exploring the mode coupling structure is that the vertices $\gamma_{a}^{b c}\left(\mathbf{k}_{1}, \mathbf{k}_{2}\right)$ can be large when the ratio $k_{1} / k_{2}$ (or its inverse) gets large. We will see that it corresponds to contributions coming from the infrared (IR) domain.

\subsection{The IR behavior for the 1-loop corrections to the power spectrum}

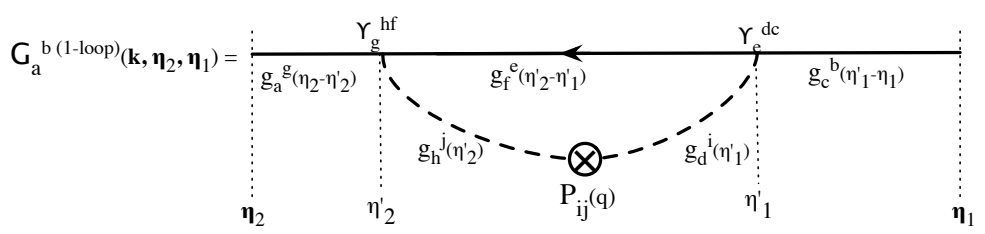

Figure 11: The one-loop correction diagram to the propagator.

Let us first consider the one-loop correction to the power spectrum. We want to compute the amplitude of the one-loop correction when the wave mode that circulates in the loop $\mathbf{q}$ is much smaller than the mode of interest, $\mathbf{k}$.

Let us consider more specially the left diagram appearing on Fig. 10 which is partly reproduced in more detail on Fig. 11. In this approximation the incoming modes from the loop are either $\mathbf{q}$ or $\mathbf{- q}$. As one assumes $q \ll k$, the modes on the horizontal line is always $\mathbf{k}$. We can then compute the expression of each of the vertices. The one on the right (at time $\eta_{1}^{\prime}$ ) then reads,

$$
\gamma_{e}^{d c} \approx \frac{1}{2} \frac{\mathbf{q} \cdot \mathbf{k}}{q^{2}} \delta_{e}^{c} \delta_{2}^{d} \delta_{\text {Dirac }}\left(\mathbf{k}-\mathbf{k}_{1}\right)
$$

Anticipating the next paragraph we can call this the eikonal limit of the vertex. We note two key properties: it is diagonal in the $c$ and $e$ indices (of the main line) and it selects only the second component of the incoming waves. The value of the other vertex can be similarly evaluated,

$$
\gamma_{g}^{h f} \approx-\frac{1}{2} \frac{\mathbf{q} \cdot(\mathbf{k}+\mathbf{q})}{q^{2}} \delta_{g}^{f} \delta_{2}^{h} \delta_{\text {Dirac }}\left(\mathbf{k}-\mathbf{k}_{1}\right) \approx-\frac{1}{2} \frac{\mathbf{q} \cdot \mathbf{k}}{q^{2}} \delta_{g}^{f} \delta_{2}^{h} \delta_{\text {Dirac }}\left(\mathbf{k}-\mathbf{k}_{1}\right)
$$

The actual computation of the diagram then requires to

- sum over all internal indices;

- integrate the intermediate time variables, $\eta_{1}^{\prime}$ and $\eta_{2}^{\prime}$, over adequate intervals;

- integrate over the angle between $\mathbf{q}$ and $\mathbf{k}$. 
The first step is now easy to perform and relies on generic properties of Green functions; the algebraic structure along the $\mathbf{k}$-line indeed reads

$$
\begin{aligned}
g_{a}^{g}\left(\eta_{2}\right. & \left.-\eta_{2}^{\prime}\right) \delta_{g}^{f} g_{f}^{e}\left(\eta_{2}^{\prime}-\eta_{1}^{\prime}\right) \delta_{e}^{c} g_{c}^{b}\left(\eta_{1}^{\prime}-\eta_{1}\right) \\
& =g_{a}^{f}\left(\eta_{2}-\eta_{2}^{\prime}\right) g_{f}^{c}\left(\eta_{2}^{\prime}-\eta_{1}^{\prime}\right) g_{c}^{b}\left(\eta_{1}^{\prime}-\eta_{1}\right) \\
& =g_{a}^{b}\left(\eta_{2}-\eta_{1}\right) .
\end{aligned}
$$

The integration over the relative angle between $\mathbf{q}$ and $\mathbf{k}$ can then be done straightforwardly:

$$
\int \mathrm{d}^{3} \mathbf{q} \frac{(\mathbf{q} \cdot \mathbf{k})^{2}}{q^{4}}=\frac{4 \pi}{3} \int q^{2} \mathrm{~d} q \frac{k^{2}}{q^{2}}
$$

We can now insert this result into the first diagram of Fig. 10. It leads to

$$
P_{a b}^{1-l o o p, \# 1}(k)=P_{a b}^{\text {lin. }}(k)\left[1-k^{2} \sigma_{d}^{2}\right]
$$

with

$$
\sigma_{d}^{2}(\eta)=\frac{4 \pi}{3} \int \mathrm{d} q\left(e^{\eta}-e^{\eta_{0}}\right)^{2} P_{22}^{\text {lin. }}(q)
$$

if the incoming velocity modes are the in linear growing mode. $\sigma_{d}$ can easily be interpreted as the r.m.s. of the 1D displacement field. A very similar calculation can be done for the second diagram of Fig. 10. Using the same approximation one gets

$$
P_{a b}^{1-l o o p, \# 2}(k)=P_{a b}^{\text {lin. }}(k)\left[1+k^{2} \sigma_{d}^{2}\right]
$$

and the two contributions actually cance ${ }^{4}$. This cancellation was first noted by Jain and Bertschinger (1996) and by Scoccimarro and Frieman (1996), In the following we explicitly show how it can be extended to all orders in perturbation theory with the help of the so called eikonal approximation (introduced in Bernardeau et al. 2012).

\subsection{The eikonal approximation}

The eikonal approximation has been used in various contexts. It originally comes from the equations of wave propagation: it is a standard approximation which leads to the laws of geometric optics. It has also been used in the context of Quantum Electro-Dynamics, in a manner very similar to the way we are going to use it, to exponentiate the effect of soft photon modes on the propagator of electrons (see for instance Abarbanel and Itzykson 1969).

In the context of gravitational dynamics, it is based on the decomposition of right hand side of eqn (77) into 2 domains, the soft domain where one of the mode is much smaller than the other one, and one hard domain where the two interacting wave-modes are of the same order. Let us assume for simplicity that the soft domain is obtained for $k_{1} \ll k_{2}$, then in the source term of (77) one should have $\mathbf{k}=\mathbf{k}_{2}$. The contribution corresponding to that domain can then be viewed as a corrective term to the linear evolution of the mode $\mathbf{k}$. In other words the motion equation can then be written,

$$
\begin{aligned}
\frac{\partial}{\partial \eta} \Psi_{a}(\mathbf{k}, \eta)+\Omega_{a}^{b}(\eta) \Psi_{b}(\mathbf{k}, \eta)-\Xi_{a}^{b}(\mathbf{k}, \eta) \Psi_{b}(\mathbf{k}, \eta) & \\
& =\left[\gamma_{a}^{b c}\left(\mathbf{k}_{1}, \mathbf{k}_{2}\right) \Psi_{b}\left(\mathbf{k}_{1}, \eta\right) \Psi_{c}\left(\mathbf{k}_{2}, \eta\right)\right]_{\mathcal{H}}
\end{aligned}
$$

\footnotetext{
${ }^{4}$ The actual calculations should be done with care in particular for a correct determination of the symmetry factors.
} 
with

$$
\Xi_{a}^{b}(\mathbf{k}, \eta) \equiv 2 \int_{\mathcal{S}} \mathrm{d}^{3} \mathbf{q} \gamma_{a}^{b c}(\mathbf{k}, \mathbf{q}) \Psi_{c}(\mathbf{q}, \eta) .
$$

The key point to make eqn (96) sensible is that in eqn (97) the domain of integration is restricted to soft momenta for which $q \ll k$, the soft domain. Conversely, on the right-hand side of eqn (96) the convolution is done excluding the soft domain, i.e. it is over hard modes or modes of comparable size.

Note that $\Xi_{a}^{b}(\mathbf{k}, \eta)$ is a random coefficient. It depends on the initial conditions but is (assumed to be) independent on the mode whose evolution we are interested in. The equation (96) can then be viewed as the motion equation of cosmological modes in a random medium with largescale modes. It allows to compute how the long wave modes alter the growth of structure. In that context the vertex value appearing in this expression corresponds to that used for the computation of the one-loop correction to the power spectrum in the IR domain in the previous paragraph,

$$
\gamma_{a}^{b c}\left(\mathbf{k}_{1}, \mathbf{q}\right) \approx \frac{1}{2} \frac{\mathbf{q} \cdot \mathbf{k}}{q^{2}} \delta_{a}^{b} \delta_{2}^{c} \delta_{\operatorname{Dirac}}\left(\mathbf{k}-\mathbf{k}_{1}\right)
$$

We restrict here our analysis to the property of a single fluid (or if there are two fluids to the adiabati $5^{5}$ modes $)$. It leads to an explicit expression for $\Xi_{a}^{b}(\mathbf{k}, \eta)$,

$$
\Xi_{a}^{b}(\mathbf{k}, \eta)=\Xi(\mathbf{k}, \eta) \delta_{a b}, \quad \Xi(\mathbf{k}, \eta) \equiv \int_{\mathcal{S}} \mathrm{d}^{3} \mathbf{q} \frac{\mathbf{k} \cdot \mathbf{q}}{q^{2}} \hat{\theta}(\mathbf{q}, \eta) .
$$

Note that only the velocity field $\hat{\theta}$ (and not the density field $\delta$ ) contributes to $\Xi_{a}{ }^{b}$. Furthermore, as $\hat{\theta}(\mathbf{x}, \eta)$ is real $\hat{\theta}(-\mathbf{q})=\hat{\theta}^{*}(\mathbf{q})$ and thus $\Xi$ is purely imaginary.

We can now explore the behavior of this new dynamical system. In particular the impact of the IR modes are now all encoded in the $\Xi_{a}^{b}(\mathbf{k}, \eta)$ coefficient and so are all encoded in the linearized equation of motion (obtained when the right hand side of eq (96) is dropped). That equation admits new Green functions $\xi_{a}{ }^{b}\left(\mathbf{k}, \eta, \eta^{\prime}\right)$ that can be explicitly computed. They satisfy the equation

$$
\left(\frac{\partial}{\partial \eta}-\Xi(\mathbf{k}, \eta)\right) \xi_{a}^{b}\left(\mathbf{k}, \eta, \eta^{\prime}\right)+\Omega_{a}^{c}(\eta) \xi_{c}^{b}\left(\mathbf{k}, \eta, \eta^{\prime}\right)=0,
$$

In the case of a single fluid, as discussed here, eqn 100 can be easily solved. Taking into account the boundary condition $\xi_{a}^{b}(\mathbf{k}, \eta, \eta)=\delta_{a}^{b}$, one obtains

$$
\xi_{a}^{b}\left(\mathbf{k}, \eta, \eta_{0}\right)=g_{a}^{b}\left(\eta, \eta_{0}\right) \exp \left(\int_{\eta_{0}}^{\eta} \mathrm{d} \eta^{\prime} \Xi\left(\mathbf{k}, \eta^{\prime}\right)\right) .
$$

The argument of the exponential is the time integral of the velocity projected along the direction $\mathbf{k}$, i.e. the displacement component along $\mathbf{k}$, that is

$$
\xi_{a}^{b}\left(\mathbf{k}, \eta, \eta^{\prime}\right)=g_{a}{ }^{b}\left(\eta, \eta_{0}\right) \exp \left(\mathrm{ik} \cdot \mathbf{d}\left(\eta, \eta^{\prime}\right)\right)
$$

where $\mathbf{d}\left(\eta, \eta^{\prime}\right)$ is the total displacement induced by the long wave modes between time $\eta^{\prime}$ and $\eta$. Note that eqn (101) is valid irrespectively of the fact that the incoming modes in $\Xi$ are in the growing mode or not.

\footnotetext{
${ }^{5}$ Non-adiabatic large-scale modes can also be considered and lead to non-trivial phenomena such as the damping of the small scale fluctuations due to large relative displacement between baryons and dark matter particles as first discussed by Tseliakhovich and Hirata (2010) and later derived within the eikonal approximation scheme (Bernardeau et al. 2013).
} 
The consequences of this result are multifold. In particular it explicitly gives the impact of the long-wave modes on the growth of structure: they are entirely captured by a phase shift in the propagator values which is proportional to $\mathbf{k . d}\left(\eta, \eta^{\prime}\right)$. If one now considers any contribution to any equal time multi-point spectrum, it is easy to see that the total phase shifts exactly cancel out such that the long wave modes have no impact on the equal time correlators. It generalizes to any order the result of the previous paragraph (see detailed derivation of this property in Bernardeau et al. 2013, Blas et al. 2013b).

The second consequence is that it is now possible to compute resumed propagators. This is at the heart of the so-called RPT and RegPT propositions described in the next section.

\subsection{The extended Galilean invariance from the equivalence principle}

The previous result is actually closely related to an invariance sometimes called the extended Galilean invariance (Kehagias and Riotto 2013, Peloso and Pietroni 2013), which actually derives from the equivalence principle as shown in Creminelli et al. (2013), which the pressureless VlasovPoisson system satisfies ${ }^{6}$. It should be clear that this invariance significantly extends that of a mere Galilean invariance as it states that the development of the gravitational instabilities in a given patch of the universe is the same irrespectively of the fact that this patch is accelerated or not. More explicitly the motion equations are invariant under the following transformations ${ }^{7}$

$$
x_{i} \rightarrow x_{i}-s_{i}(\eta), \quad u_{i} \rightarrow u_{i}+\frac{\mathrm{d}}{\mathrm{d} \eta} s_{i}(\eta)
$$

where $\mathbf{s}(\eta)$ is an arbitrary time dependent vector. Under this transformation the linear propagator of the theory is precisely changed into,

$$
g_{a}^{b}\left(\eta, \eta^{\prime}\right) \rightarrow g_{a}^{b}\left(\eta, \eta^{\prime}\right) \exp \left(\mathrm{ik} \cdot\left[\mathbf{s}(\eta)-\mathbf{s}\left(\eta^{\prime}\right)\right]\right)
$$

which is reminiscent of eqn 102 . In other words the adiabatic long wave modes can entirely be absorbed by an extended Galilean transformation leaving no imprint on equal time correlators.

Interestingly though, the existence of a transformation under which the equations are invariant leads to so-called Ward identities the simplest of which we reproduce here,

$$
\begin{aligned}
\lim _{q \rightarrow 0} B(\mathbf{q}, \mathbf{k},-\mathbf{k}-\mathbf{q} ; \eta & \left., \eta^{\prime}, \eta^{\prime \prime}\right)= \\
& -\frac{\mathbf{q} \cdot \mathbf{k}}{q^{2}} P^{\text {lin. }}(q ; \eta, \eta) P^{\text {lin. }}\left(k ; \eta^{\prime}, \eta^{\prime \prime}\right)\left(e^{\eta^{\prime}-\eta}-e^{\eta^{\prime \prime}-\eta}\right)
\end{aligned}
$$

where $P^{\text {lin. }}\left(k ; \eta, \eta^{\prime}\right)$ is the linear unequal time power spectrum taken between time $\eta$ and $\eta^{\prime}$ and $B\left(\mathbf{k}_{1}, \mathbf{k}_{2},-\mathbf{k}_{1}-\mathbf{k}_{2} ; \eta_{1}, \eta_{2}, \eta_{3}\right)$ is the unequal time bispectrum, i.e.

$$
\begin{aligned}
& \left\langle\delta\left(\mathbf{k}_{1}, \eta_{1}\right) \delta\left(\mathbf{k}_{2}, \eta_{2}\right) \delta\left(\mathbf{k}_{3}, \eta_{3}\right)\right\rangle= \\
& \left.\left.\delta_{\text {Dirac }}\left(\mathbf{k}_{1}+\mathbf{k}_{2}+\mathbf{k}_{3}\right) B\left(\mathbf{k}_{1}, \mathbf{k}_{2},-\mathbf{k}_{1}-\mathbf{k}_{2} ; \eta_{1}, \eta_{2}, \eta_{3}\right)\right)\right\rangle .
\end{aligned}
$$

The identity 105 involves unequal time correlators and as such is probably very difficult to implement in actual observations.

\footnotetext{
2000).

${ }^{6}$ Interestingly the same invariance is satisfied by the Navier-Stokes equations with constant density Pope T'To get a fully valid transformation one should also change the gravitational potential gradient in such a way that the source term of the Euler equation is changed in $f_{i} \rightarrow f_{i}+\frac{\mathrm{d}^{2}}{\mathrm{~d} \eta^{2}} s_{i}(\eta)+\frac{1}{2} \frac{\mathrm{d}}{\mathrm{d} \eta} s_{i}(\eta)$ which leaves the Poisson equation unchanged.
} 


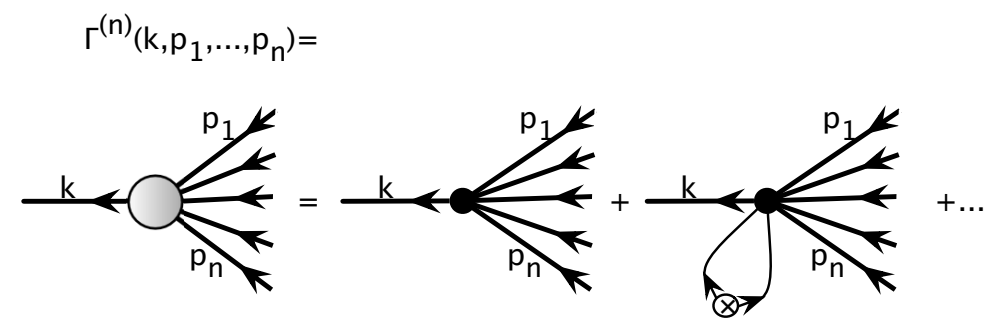

Figure 12: Representation of the first two terms of the multi-point propagator $\Gamma_{g}^{(n)}$ in a perturbative expansion. $\Gamma_{g}^{(n)}$ represents the average value of the emerging nonlinear mode $\mathbf{k}$ given $n$ initial modes in the linear regime. Here we show the first two contributions: tree-level and one-loop. Note that each object represents a collection of (topologically) different diagrams: each black dot represents a set of trees that connect respectively $n+1$ lines for the first term, $n+2$ for the second.

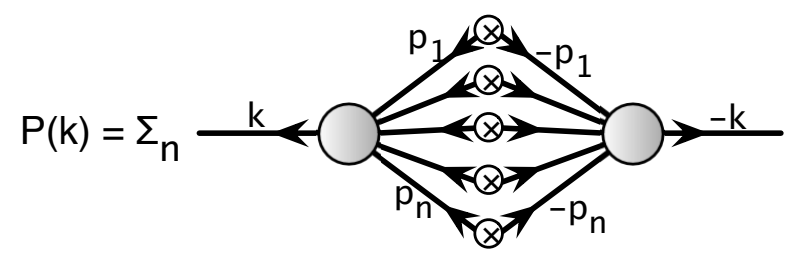

Figure 13: Representation of the resummation rule given by eqn (110). For Gaussian initial conditions, the power spectrum can be seen as a sum of squares of $\Gamma^{(p)}$ functions.

\section{The $\Gamma$ - expansion}

\subsection{The general formalism and theorem}

The so-called $\Gamma$-expansion is a very general result that can be applied to any nonlinear field transformation. It has been explicitly demonstrated in the context of nonlinear gravitational dynamics (Bernardeau et al. 2012) but results regarding biasing schemes can also be derived in the very same framework (McDonald 2006; Schmidt et al. 2013). So let us consider a field $g(\mathbf{x}, \eta)$ and its Fourier modes $g(\mathbf{k}, \eta)$. Let us now assume that $g(\mathbf{k}, \eta)$ can be expanded in terms of an initial Gaussian random field $\zeta(\mathbf{k})$,

$$
g(\mathbf{k}, \eta)=\sum_{n} \int \frac{\mathrm{d}^{3} \mathbf{k}_{1}}{(2 \pi)^{3 / 2}} \ldots \frac{\mathrm{d}^{3} \mathbf{k}_{n}}{(2 \pi)^{3 / 2}} \delta_{D}\left(\mathbf{k}-\sum_{i} \mathbf{k}_{i}\right) g_{n}\left(\mathbf{k}_{1}, \ldots, \mathbf{k}_{n}, \eta\right) \zeta\left(\mathbf{k}_{1}\right) \ldots \zeta\left(\mathbf{k}_{n}\right),
$$

where the $g_{n}$ functions are time dependent functions. In particular $g_{1}(\eta, \mathbf{k})$ is nothing but the linear solution for that particular fluid. One question that one might want to ask is how the growth rate of the fluctuation is actually affected by the fluctuations. Multipoint propagators are precisely defined as the ensemble averages (over fluctuations in the medium) of the infinitesimal response of the system to an initial perturbation. More precisely we can define the $\Gamma^{(p)}$ functions as,

$$
\frac{1}{p !}\left\langle\frac{\delta g(\mathbf{k}, \eta)}{\delta \zeta\left(\mathbf{q}_{1}\right) \ldots \delta \zeta\left(\mathbf{q}_{p}\right)}\right\rangle \equiv \delta_{\operatorname{Dirac}}\left(\mathbf{k}-\sum_{r=1}^{p} \mathbf{q}_{r}\right) \Gamma_{g}^{(p)}\left(\mathbf{q}_{1}, \ldots, \mathbf{q}_{p}\right)
$$




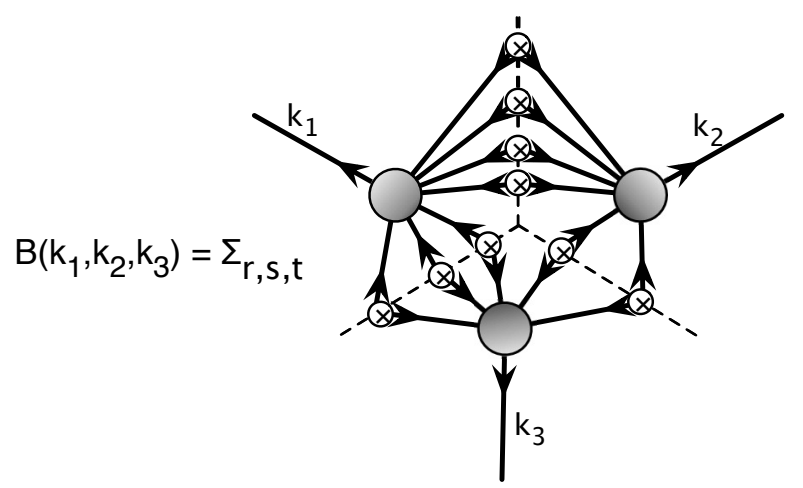

Figure 14: Representation of the resummation rule given by eqn (111). For Gaussian initial conditions, the bispectrum can be seen as a sum of product of $\Gamma^{(p)}$ functions.

To obtained the values of $\Gamma^{(p)}$ out of the $g_{n}\left(\eta, \mathbf{k}_{1}, \ldots, \mathbf{k}_{n}\right)$ function, in each term starting with $n=p$ in eq (107) one should take out $p$ modes and compute the ensemble average of the remaining terms. A diagrammatic representation of this procedure can be found on Fig. 12 . The $\Gamma^{(p)}$ function are thus nonlinear objects on their own. Note a useful property,

$$
\Gamma_{g_{1}}^{(p)}\left(-\mathbf{q}_{1}, \ldots,-\mathbf{q}_{p}\right)=\Gamma_{g_{1}}^{(p)}\left(\mathbf{q}_{1}, \ldots, \mathbf{q}_{p}\right)
$$

from parity symmetry of the field $g$.

The key result explicitly demonstrated in Bernardeau et al. (2012) is then that the power spectrum of the $g$ field, or actually the cross spectra of any of two such fields $g_{1}$ and $g_{2}$, can then be reconstructed out of the multipoint propagators following the formulae,

$$
\begin{aligned}
& \left\langle g_{1}(\mathbf{k}) g_{2}\left(\mathbf{k}^{\prime}\right)\right\rangle=\delta_{\text {Dirac }}\left(\mathbf{k}+\mathbf{k}^{\prime}\right) \sum_{p} p ! \int \mathrm{d}^{3} \mathbf{q}_{1} \ldots \mathrm{d}^{3} \mathbf{q}_{p} \\
& \quad \times \delta_{\text {Dirac }}\left(\mathbf{k}-\mathbf{q}_{1 \ldots p}\right) \Gamma_{g_{1}}^{(p)}\left(\mathbf{q}_{1}, \ldots, \mathbf{q}_{p}\right) \Gamma_{g_{2}}^{(p)}\left(\mathbf{q}_{1}, \ldots, \mathbf{q}_{p}\right) P_{\zeta}\left(q_{1}\right) \ldots P_{\zeta}\left(q_{p}\right),
\end{aligned}
$$

when $\zeta$ is Gaussian distributed. Note that when one considers the spectrum of a given field, the formulae is then a sum of square terms making each contribution positive. As it is clear from the very beginning this is a very general construction which is valid in particular for the evolved density and velocity fields. Note that an extension of this relation exists for non-Gaussian initial conditions (Bernardeau et al. 2010).

The previous construction for the spectra can actually be extended to any high-order spectra. The formal expression for the general term is a bit cumbersome. However, for the bispectrum it is still possible to write its formal expression,

$$
\begin{aligned}
& \left\langle g_{1}\left(\mathbf{k}_{1}\right) g_{2}\left(\mathbf{k}_{2}\right) g_{c}\left(\mathbf{k}_{3}\right)\right\rangle=\sum_{r, s, t}\left(\begin{array}{c}
r+s \\
r
\end{array}\right)\left(\begin{array}{c}
s+t \\
s
\end{array}\right)\left(\begin{array}{c}
t+r \\
t
\end{array}\right) r ! s ! t ! \\
& \quad \int \Pi_{i=1}^{r} \mathrm{~d}^{3} \mathbf{q}_{i} P_{\zeta}\left(q_{i}\right) \Pi_{j=1}^{s} \mathrm{~d}^{3} \mathbf{q}_{j} P_{\zeta}\left(q_{j}\right) \Pi_{k=1}^{t} \mathrm{~d}^{3} \mathbf{q}_{k} P_{\zeta}\left(q_{k}\right) \\
& \quad \times \delta_{\text {Dirac }}\left(\mathbf{k}_{1}-\mathbf{q}_{1 \ldots r}-\mathbf{q}_{1 \ldots s}^{\prime}\right) \delta_{\operatorname{Dirac}}\left(\mathbf{k}_{2}+\mathbf{q}_{1 \ldots s}^{\prime}-\mathbf{q}_{1 \ldots t}^{\prime \prime}\right) \delta_{\operatorname{Dirac}}\left(\mathbf{k}_{3}+\mathbf{q}_{1 \ldots t}^{\prime \prime}+\mathbf{q}_{1 \ldots r}\right) \\
& \quad \times \Gamma_{a}^{(r+s)}\left(\mathbf{q}_{1}, \ldots, \mathbf{q}_{r}, \mathbf{q}_{1}^{\prime}, \ldots, \mathbf{q}_{s}^{\prime}\right) \Gamma_{b}^{(s+t)}\left(-\mathbf{q}_{1}^{\prime}, \ldots,-\mathbf{q}_{s}^{\prime}, \mathbf{q}_{1}^{\prime \prime}, \ldots, \mathbf{q}_{t}^{\prime \prime}\right) \\
& \quad \times \Gamma_{c}^{(t+r)}\left(-\mathbf{q}_{1}^{\prime \prime}, \ldots,-\mathbf{q}_{t}^{\prime \prime},-\mathbf{q}_{1}, \ldots,-\mathbf{q}_{r}\right) .
\end{aligned}
$$


This reconstruction is illustrated on Fig. 14 where it is shown how the product of three $\Gamma$-functions can be "glued" together for the computation of bispectra.

\subsection{The case of gravitational dynamics}

We now move to the application of this formalism to the cosmological density and velocity fields. To be exact one should take into account the index structure of the theory which we overlooked in the previous paragraph. For instant the $\Gamma^{(1)}$ has now an algebraic structure as,

$$
\left\langle\frac{\delta \Psi_{a}\left(\mathbf{k}, \eta_{f}\right)}{\delta \Psi_{b}\left(\mathbf{k}^{\prime}, \eta_{i}\right)}\right\rangle=\delta_{\text {Dirac }}\left(\mathbf{k}-\mathbf{k}^{\prime}\right) \Gamma_{a}^{(1) b}\left(k, \eta_{f}, \eta_{i}\right),
$$

where we also allow ourselves to consider the initial time in eqn (107) as a free parameter. Usually this quantity is noted,

$$
G_{a}^{b}\left(k, \eta_{f}, \eta_{i}\right) \equiv \Gamma_{a}^{(1) b}\left(k, \eta_{f}, \eta_{i}\right) .
$$

It is nothing but the generalization of the linear propagator. The idea of replacing the linear propagator by a resumed propagator is at the heart of the so-called RPT approach (Crocce and Scoccimarro 2006b, Crocce and Scoccimarro 2006a).

The expression for $G_{a}^{b}$ can be computed order by order in perturbation theory. Such results can be obtained from a formal expansion of $\Psi_{a}(\mathbf{k}, \eta)$ with respect to the initial field,

$$
\Psi_{a}(\mathbf{k}, \eta)=\sum_{n=1}^{\infty} \Psi_{a}^{(n)}(\mathbf{k}, \eta)
$$

with

$$
\Psi_{a}^{(n)}(\mathbf{k}, \eta)=\mathcal{F}_{a}^{(n) b_{1} b_{2} \ldots b_{n}}\left(\mathbf{k}_{1}, \ldots, \mathbf{k}_{n} ; \eta\right) \Phi_{b_{1}}\left(\mathbf{k}_{1}\right) \ldots \Phi_{b_{n}}\left(\mathbf{k}_{n}\right)
$$

where $\mathcal{F}^{(n)}$ are fully symmetric functions of the wave-vectors. Note that these functions have in general a non-trivial time dependence because they also include sub-leading terms in $\eta$. Their fastest growing term is of course given by the well known $\left\{F_{n}, G_{n}\right\}$ kernels in PT (assuming growing mode initial conditions),

$$
\begin{aligned}
& \mathcal{F}_{a}^{(n) b_{1} b_{2} \ldots b_{n}}\left(\mathbf{k}_{1}, \ldots, \mathbf{k}_{n} ; \eta\right) u_{b_{1}} \ldots u_{b_{n}}= \\
& \delta_{\text {Dirac }}\left(\mathbf{k}-\mathbf{k}_{1 \ldots n}\right) \exp (n \eta)\left(F_{n}\left(\mathbf{k}_{1}, . ., \mathbf{k}_{n}\right), G_{n}\left(\mathbf{k}_{1}, . ., \mathbf{k}_{n}\right)\right)^{T}
\end{aligned}
$$

whose two elements correspond to the density or velocity divergence fields respectively.

\subsection{The RPT formulation}

We are now in position to give the explicit form of the RPT proposition described by Crocce and Scoccimarro (2006b). It is based on the construction of the propagator $G_{a}^{b}(k, \eta)$ that encompasses both its full one-loop correction and the effect of the long-wave modes computed at all orders. The explicit form adopted in the original paper is too cumbersome to be re-derived here in detail but a form that shares its properties can easily be obtained. The impact of the long-wave modes can indeed be all incorporated with the help of the eikonal approximation where the "naked' Green function, $g_{a}{ }^{b}\left(\eta, \eta^{\prime}\right)$, is transformed into the "dressed" one, $\xi_{a}{ }^{b}\left(\eta, \eta^{\prime}\right)$. Then armed with the results from Section 6.2 we can first compute the ensemble average of $\xi_{a}{ }^{b}(\mathbf{k}, \eta)$ with respect to 


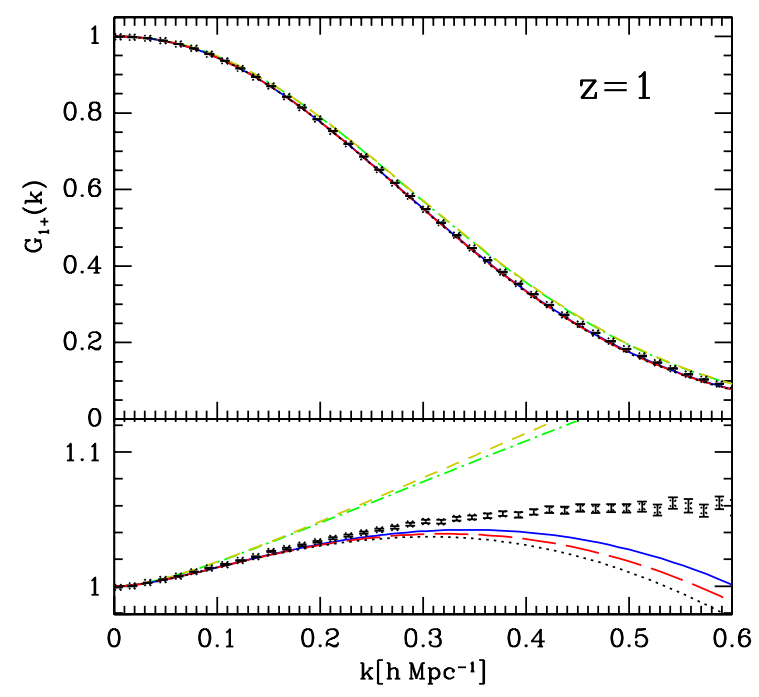

Figure 15: The predicted resumed propagator (lines) compared with numerical simulations (symbols with error bars) at $z=1$. The top panel shows the $G_{1+}(k)$ as a function of $k$ and the bottom panel shows $G_{1+}(k) \exp \left(-k^{2} \sigma_{d}^{2} / 2\right)$. The dashed and dot-dashed lines are the RPT and RegPT form at 1-loop order. The bottom lines shows the 2-loop predictions.

the long-wave modes. When those modes are assumed to be in the linear regime and therefore to be Gaussian distributed, the use of the relation (74), leads to,

$$
\left\langle\xi_{a}^{b}(\mathbf{k}, \eta)\right\rangle=g_{a}^{b}\left(\eta, \eta^{\prime}\right) \exp \left[-\frac{1}{2} k^{2} \sigma_{d}^{2}\left(\eta, \eta^{\prime}\right)\right],
$$

where $\sigma_{d}\left(\eta, \eta^{\prime}\right)$ is given by,

$$
\sigma_{d}^{2}\left(\eta, \eta^{\prime}\right)=\frac{1}{3}\left\langle\mathbf{d}^{2}\left(\eta, \eta^{\prime}\right)\right\rangle=\left(e^{\eta}-e^{\eta^{\prime}}\right)^{2} \int \frac{\mathrm{d}^{3} \mathbf{q}}{3 q^{2}} P^{\text {lin. }}(q) .
$$

This resummation result is the key result on which the RPT scheme is based. It is furthermore possible to include the full contribution of the one-loop contribution as given by the diagram of Fig. 11. Indeed this diagram itself can be computed within the eikonal framework and it leads to

$$
G_{a}^{1-\operatorname{loop} b}\left(\mathbf{k}, \eta, \eta^{\prime}\right) \rightarrow G_{a}^{1-\operatorname{loop} b}\left(\mathbf{k}, \eta, \eta^{\prime}\right) \exp \left(\mathrm{ik.d}\left(\eta, \eta^{\prime}\right)\right)
$$

the ensemble average of which over the long-wave modes can also be taken. A possible global form in then the following (Bernardeau et al. 2012),

$$
\begin{aligned}
G_{a}^{\mathrm{reg} b}\left(k, \eta_{f}, \eta_{i}\right)= & {\left[g_{a}^{b}\left(\eta, \eta^{\prime}\right)+\delta G_{a}^{1-\operatorname{loop} b}\left(k, \eta, \eta^{\prime}\right)+\frac{1}{2} k^{2} \sigma_{d}^{2}\left(\eta, \eta^{\prime}\right) g_{a}^{b}\left(\eta, \eta^{\prime}\right)\right] } \\
& \times \exp \left(-\frac{k^{2} \sigma_{d}^{2}\left(\eta, \eta^{\prime}\right)}{2}\right),
\end{aligned}
$$

which has the expected behavior for both the large $k$ and in the low $k$ domains.

On Fig. 15 we show the performance of the RPT type prediction on the behavior of the propagator. The measured values of $G_{1+}(k) \equiv G_{1}^{b}(k) u_{b}^{(+)}$are compared with the analytical predictions up to 2 loop order. The RPT formulation used the 1-loop order predictions only. 

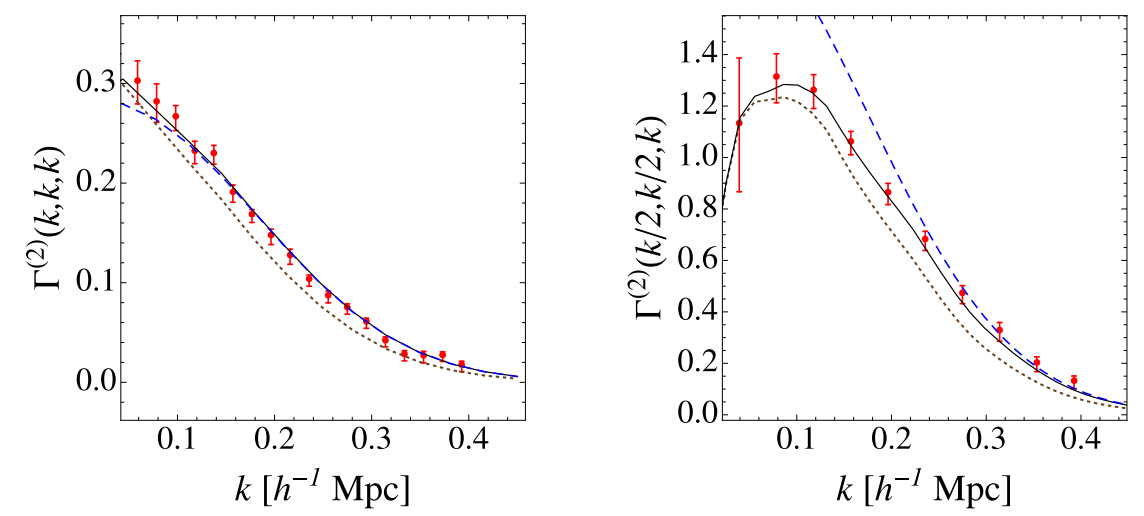

Figure 16: The predicted resumed $\Gamma^{(2)}$ for two different configurations up to one loop order (solid lines) compared with numerical simulations (symbols with error bars). The dotted lines are the tree oder predictions and the dashed lines the one-loop order neglecting the binning effects.

\subsection{The MPTbreeze and RegPT formulation}

Other resummation schemes, MPTbreeze (Crocce et al. 2012a) and RegPT (Taruya et al. 2012) proposed afterwards take full advantage 8 of the $\Gamma$-expansion presented in the previous section. It is based on a similar construction to 120 applied to the $p$-point propagator. More specifically the following form can be used,

$$
\begin{aligned}
& \Gamma_{a}^{\mathrm{reg} b_{1} \ldots b_{p}}\left(\mathbf{k}_{1}, \ldots, \mathbf{k}_{p}, \eta, \eta^{\prime}\right)= \\
& {\left[\Gamma_{a}^{\mathrm{tree} b_{1} \ldots b_{p}}\left(\mathbf{k}_{1}, \ldots, \mathbf{k}_{p}, \eta, \eta^{\prime}\right)+\delta \Gamma_{a}^{1-\operatorname{loop} b_{1} \ldots b_{p}}\left(\mathbf{k}_{1}, \ldots, \mathbf{k}_{p}, \eta, \eta^{\prime}\right)\right.} \\
&+\left.\frac{1}{2} k^{2} \sigma_{d}^{2}\left(\eta, \eta^{\prime}\right) \Gamma_{a}^{\text {tree } b_{1} \ldots b_{p}}\left(\mathbf{k}_{1}, \ldots, \mathbf{k}_{p}, \eta, \eta^{\prime}\right)\right] \exp \left(-\frac{k^{2} \sigma_{d}^{2}\left(\eta, \eta^{\prime}\right)}{2}\right)
\end{aligned}
$$

where $k=\left|\mathbf{k}_{1}+\ldots+\mathbf{k}_{p}\right|$. By construction this form is such that it has both the correct one-loop correction and the correct large- $k$ behavior. A comparison for the $p=2$ case is presented on Fig. 16.

It can then be incorporated in a $\Gamma$-expansion scheme. In practice the MPTbreeze and RegPT codes exploit this formalism up to 2-loop order, that is $G_{a}{ }^{b}(k, \eta)$ is computed to to 2-loop order (1-loop order only for MPTbreeze), $\Gamma_{a}^{(2) b_{1} b_{2}}$ to 1-loop order and $\Gamma_{a}^{(3) b_{1} b_{2} b_{3}}$ at tree order.

Note that all these approaches exhibit similar properties. In particular the predicted power spectra are damped at large $k$ with an overall factor of the form, $\exp \left(-k^{2} \sigma_{d}^{2}\right)$. This result is at variance with one consequence of the extended Galilean invariance, that is that equal-time spectra should be independent on the long-wave modes that participate in the values of $\sigma_{d}$. At two-loop order though the lowest order dependence on $\sigma_{d}$ is $k^{6} \sigma_{d}^{6}$ (all lower orders in $\sigma_{d}$ cancel out). The remaining dependence that we have in these schemes actually signal the validity range of their predictions.

\subsection{The performances of perturbation theory at NNLO}

To conclude this section we present the performances of the perturbation theory at two-loop order. On Fig. 17 we present the performances of the RegPT up to 2-loop order compared

\footnotetext{
${ }^{8}$ They also have the advantage to be faster to compute.
} 

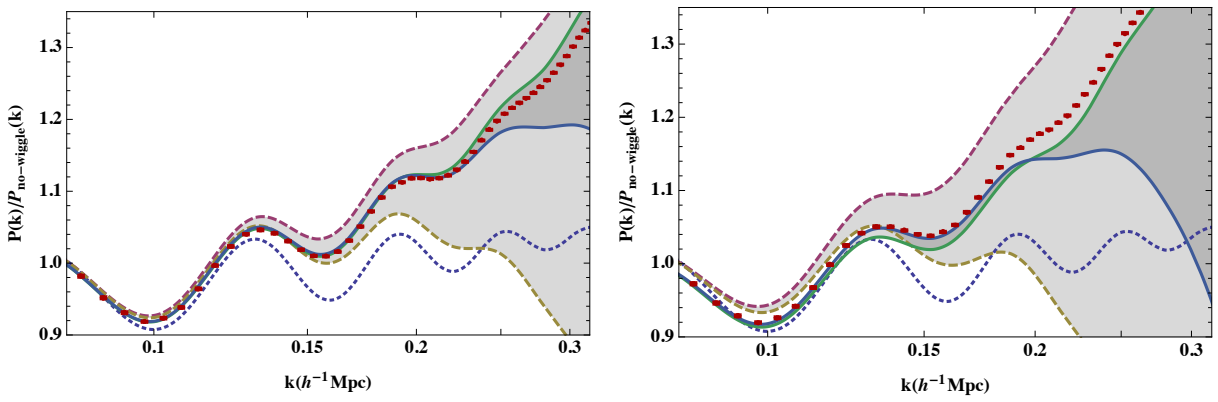

Figure 17: Comparison of PT results with $N$-body results for the power spectrum at $z=1$ (left panel) and $z=0.35$ (right panel). The dotted line is the linear prediction; the dashed lines are the standard PT and RegPT NLO predictions and the solid lines the NNLO predictions. The grey area show the departure between these predictions at one-loop order (light grey) and 2-loop order (darker grey).

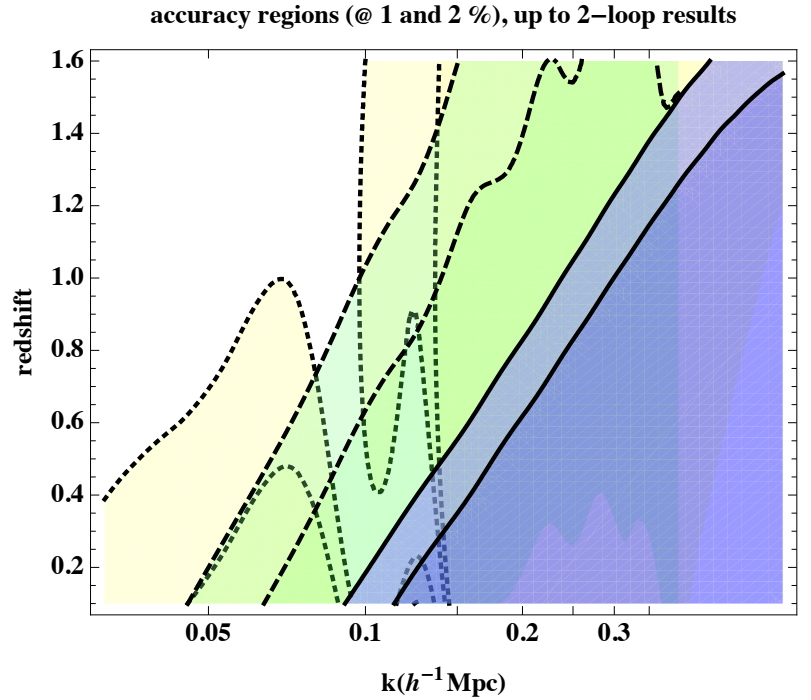

Figure 18: A tentative chart of the accuracy domain of the PT results with contour plots of the 1 and $2 \%$ accuracy region for the linear, NLO, and NNLO predictions for the power spectrum. The calculations have been made for the WMAP5 cosmological parameters. 
to standard PT and compared to numerical results. The solid lines show the two-loop order predictions for the standard PT and the RegPT schemes. They agree with another up to wavemodes that are significantly larger than the linear theory validity regime. They also extent significantly the validity regime of the one-loop results. This is particular striking at redshift 1 and above.

These findings are summarized on Fig. 18 which tentatively charts the performances of the linear, one and two-loop predictions. The dotted lines correspond to the $1 \%$ and $2 \%$ accuracy lines of the linear theory. The contour lines are obtained from a comparison between the linear and the standard two-loop results. The dashed lines show the same results for the standard oneloop predictions and the solid lines show an estimate of those lines from a consistency comparison between different two-loop schemes.

\section{Mode coupling structure}

We are now interested more explicitly in the coupling structure of the terms that contribute to the loop corrections to the power spectra. In the previous sections we proved that they were safe from infrared divergences. One of the aim of this section is to explore in more detail their ultra-violet (UV) convergence properties.

\subsection{Scalings in the long wave mode regime}

Before we detail the converging properties of the loop diagrams, let us first make a simple remark regarding the $\Gamma$-expansion. One of the nice feature of this approach is that it tends to hierarchize the contribution in $k$ range. Indeed the lowest order contribution in $k$ from the loop corrections to the propagator (first diagram in Fig. 11p is in $k^{2}$ whereas the lowest contribution from the second diagram is in $k^{4}$. This basic property will extend to all orders in the sense that the $p=1$ term in the sum 110 will contain order $k^{2}$ corrections, and $p \leq 2$ terms will contain order $k^{4}$ terms. As we will see in the following this behavior is intimately related to the UV behavior of the theory: as the vertex coefficients are homogeneous in wave modes, large powers of $k$ correspond from better converging contributions in the UV domain.

\subsection{Kernels and integrands}

In order to better visualize these properties, let us define the functions $K_{a b}(k, q)$ as the kernels describing the linear response of the non-linear power spectrum to the linear power spectrum in such a way that,

$$
\delta P_{a b}^{\sharp}(k)=\int \frac{\mathrm{d} q}{q} K_{a b}^{\sharp}(k, q) P^{\text {lin. }}(q) .
$$

The kernels can actually be considered for any approximate scheme, or any any diagram contributing to the power spectrum. Note that the kernel functions depend themselves a priori on the initial power spectrum: at one-loop order $K_{a b}^{1-\operatorname{loop}}(k, q)$ is just a $\delta_{\text {Dirac }}$ function, it is linear in the linear power spectrum at one loop order, etc.

These functions give, for each order, the impact of a linear mode $q$ on the amplitude of the late time mode $k$ we are interested in. In particular it tells how the small-scale modes affect the largescale modes under consideration. In the following we will focus our interest in understanding the high- $q$ behavior of the kernel functions $K_{a b}(k, q)$ for the propagators. They were considered in detail in Bernardeau et al. (2012) and in Blas et al. (2013a) up to three-loop order.

In Fig. 19 we show the shape of the propagator kernel functions at one, two-loop and threeloop order for $k=0.1 \mathrm{~h} / \mathrm{Mpc}$ (in the latter case it was obtained from numerical simulations). 


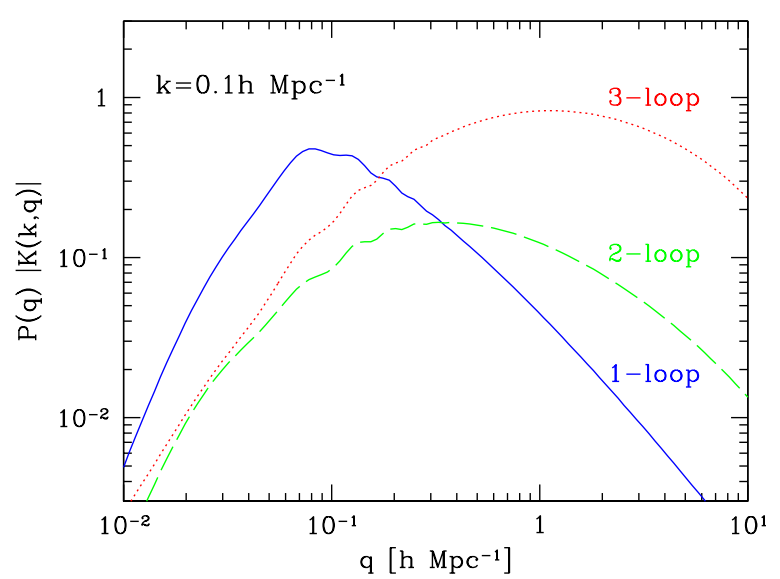

Figure 19: The shape of the kernel functions (actually the integrand in eqn 122 for the propagator $P^{\text {lin. }}(q) K_{1+}^{1-\text { loop }}(k, q)$ (blue solid line), $P^{\text {lin. }}(q) K_{1+}^{2-\text { loop }}(k, q)$ (green dashed line) for $k=0.1 \mathrm{~h} / \mathrm{Mpc}$ and $P^{\text {lin. }}(q) K_{1+}^{3-\text { loop }}(k, q)$ (red dotted line) as a function of $q$ for $z=0.5$ for the density component and for linear growing modes, from Bernardeau et al. (2012), A similar plot can be found in Blas et al. (2013a) for the power spectrum.

The solid line corresponds to the one-loop expression. As can be seen it is rather peaked at $q \approx k$ and we more explicitly have

$$
K_{1+}^{1-\text { loop }}(k, q) P^{\text {lin. }}(q)=\frac{176 \pi}{315} k^{2} q P^{\text {lin. }}(q) \text { for } q \gg k .
$$

Note that convergence of the 1-loop contribution is then ensured for a spectral index smaller than -1 .

At two-loop order, the behavior is qualitatively different. The function peaks rather for $q=0.5 h / \mathrm{Mpc}$, irrespectively of the value for $k$ (when $k<0.5 h / \mathrm{Mpc}$ ). We note that,

$$
K_{1+}^{2-\text { loop }}(k, q) P_{0}(q) \sim k^{2} q^{2} P_{0}(q) \text { for } q \gg k,
$$

so that the convergence is now secured for a spectral index smaller than -2 only.

\subsection{General convergence properties in the UV demain}

Let us now generalize these results. As mentioned in Scoccimarro and Frieman (1996) the total gradient nature of the coupling terms in the motion equations implies that,

$$
\mathcal{F}_{a}^{(n)}\left(\mathbf{q}_{1}, \ldots, \mathbf{q}_{n}\right) \sim \frac{\left|\sum_{j} q_{j}\right|^{2}}{q_{i}^{2}} \text { when } q_{i} \gg\left|\sum_{j} q_{j}\right|,
$$

whenever one of the $q_{i}$ is much larger than the sum. This can be seen at an elementary level on the properties of the vertex functions that vanish when the sum of the argument goes to 0 . The 
property 125 has direct consequences on the properties of the loop corrections. The $p$-loop correction to the propagator takes indeed the form,

$$
G^{\mathrm{p}-\text { loop }}(k, \eta)=(4 \pi)^{p} \int q_{1}^{2} \mathrm{~d} q_{1} \ldots q_{p}^{2} \mathrm{~d} q_{p} P_{0}\left(q_{1}\right) \ldots P_{0}\left(q_{p}\right) \mathcal{F}^{\mathrm{p}-\text { loop }}\left(k, q_{1}, \ldots, q_{p}\right)
$$

with

$$
\mathcal{F}\left(k, q_{1}, \ldots, q_{p}\right)=\frac{k^{2}}{\sum_{i} q_{i}^{2}} \alpha_{p}\left(k / q_{1}, \ldots, k / q_{p}\right)
$$

where $\alpha\left(k / q_{1}, \ldots, k / q_{p}\right)$ is finite in all regimes. This property determines the converging properties of the $p$-loop correction to the propagator. Clearly the converging properties of the loop corrections deteriorate. It can easily be shown that formally, for a power law spectrum of index $n_{s}$, the convergence is ensured only when,

$$
n_{s}<-3+\frac{2}{p}
$$

leading to a greater sensitivity to the large wave-modes as one increases the loop order ${ }^{9}$

Note also that the other diagrams (those coming from the $\Gamma$ - expansion) are generally better behaved. The reason is that the property 125 is then to be applied two times (on each side) gaining an overall factor $1 / q^{2}$ for the convergence in the UV domain (hence the $k^{4}$ contribution mentioned in the previous subsection). The loop contributions that are most sensitive to the UV domain are therefore those contributing to the propagator.

Unfortunately, at low redshift it implies that a regularization procedure in the UV domain should be used in order to make the contributions of these terms always realistic. This need is marginal for the two-loop contributions at low redshift, it is essential if we ever want to include the three-loop diagrams.

Let us mention possible solutions to this problem,

- partial resummation of higher order terms could provide us with a self-consistent regularization scheme (in a similar way to the IR domain). A possible solution has been recently put forward in Blas et al. (2013a) with the help of the Padé ansatz. Furthermore other resummation schemes mentioned in the introduction could lead to possible solutions as they exploit alternative series resummations;

- a reformulation of PT with other field combinations (see next section) could lead to kernels that are less sensitive to the UV modes;

- regularization could be associated from the development multi-flow regimes. In such cases none of the previous methods would work and one should rely on other type of approaches. For instance it was advocated that the impact of the small scale physics could be captured with an effective theory approach where effective anisotropic pressure terms are introduced in the fluid (Pietroni et al. 2012, Carrasco et al. 2012);

- it is eventually always possible to rely on numerical results to actually measure the kernel functions (although I am not aware of any attempt to do so in practice).

\footnotetext{
${ }^{9}$ But note that for the concordant model it converges at all order.
} 


\section{Alternative perturbation theory schemes}

It is to be noted that there is not a single way of doing perturbation theory. Indeed the choice of fields to represent the cosmic quantities is not unique. It is always possible to change into linearly related fields, for instance the potential instead of the density contrast, or the velocity potential instead of the velocity divergence, but it does not change the structure of the perturbation series. A more dramatic change is to introduce nonlinear transforms of the field. A straightforward example is to replace the peculiar velocity field by the momentum field, $\mathbf{p}(\mathbf{x}, \eta)=\rho(\mathbf{x}, \eta) \mathbf{u}(\mathbf{x}, \eta)$ (as exploited in a series of papers applied to the redshift space distortions starting with Seljak and McDonald 2011). It makes the continuity equation very simple, the divergence of $\mathbf{p}$ is the time derivative of the density to all orders but it makes the Euler equation a more cumbersome to manipulate. In particular the momentum field is no more potential to all orders.

An even more dramatic transformation is a change of the coordinate system itself. This is what the Lagrangian approach does. This is a very popular, and useful, approach that we present in more detail in the following.

\subsection{Lagrangian PT}

Perturbation Theory calculations in Lagrangian coordinates deserve a special attention as it has been advocated as a very efficient approach for a long time (see for instance Zel'dovich 1970. Buchert 1992; Bouchet et al. 1995; Buchert 1993; Buchert 1994; Matsubara 2008).

Here the fundamental quantity is the displacement field, $\Psi(\mathbf{q})$, that relates the final position of each particle to its initial position (that we will denote $\mathbf{q}$ in this section),

$$
\mathbf{x}=\mathbf{q}+\Psi(\mathbf{q})
$$

The motion equation for a particle is simple. It simply comes from the expression of the acceleration. Taking the divergence of this equation leads to the form,

$$
\partial_{x_{i}}\left[\psi_{i}^{\prime \prime}(\mathbf{q})+\left(\frac{3 \Omega_{m}}{2 f_{+}^{2}}-1\right) \psi_{i}^{\prime}(\mathbf{q})\right]=\frac{3 \Omega_{m}}{2 f_{+}^{2}} \delta(\mathbf{q}),
$$

where a prime is the derivative with respect to $\eta$ and $\delta(\mathbf{q})$ is the density contrast at the location occupied by the particles that were initially at position q. Note that in full generality the source term of this equation should take into account all flows that have reached position $\mathbf{q}+\Psi(\mathbf{q})$. In the single flow approximation, there is precisely only one flow. The density at this location is given by the Jacobian of the transform from coordinates $\mathbf{q}$ to $\mathbf{x}$,

$$
1+\delta(\mathbf{q})=\frac{1}{J(\mathbf{q})}=\frac{1}{\left|\left(\frac{\partial \mathbf{x}}{\partial \mathbf{q}}\right)\right|} .
$$

The motion equation can then be written

$$
J(\mathbf{q}) \partial_{x_{i}}\left[\psi_{i}^{\prime \prime}(\mathbf{q})+\left(\frac{3 \Omega_{m}}{2 f_{+}^{2}}-1\right) \psi_{i}^{\prime}(\mathbf{q})\right]=\frac{3 \Omega_{m}}{2 f_{+}^{2}}(J(\mathbf{q})-1) .
$$

The Jacobian of the transform can easily be expressed in terms of the derivatives of the displacement field,

$$
J(\mathbf{q})=\left|\operatorname{det}\left(\frac{\partial x_{i}}{\partial q_{j}}\right)\right|
$$


Moreover in the single flow approximation the absolute value can be dropped, the sign of the determinant is changing for each shell crossing. The determinant of the matrix $\partial x_{i} / \partial q_{j}$ is then given by

$$
J(\mathbf{q})=\frac{1}{6} \epsilon^{i j k} \epsilon^{i^{\prime} j^{\prime} k^{\prime}}\left(\delta_{i i^{\prime}}+\psi_{i, i^{\prime}}(\mathbf{q})\right)\left(\delta_{j j^{\prime}}+\psi_{j, j^{\prime}}(\mathbf{q})\right)\left(\delta_{k k^{\prime}}+\psi_{k, k^{\prime}}(\mathbf{q})\right)
$$

and appears to be a polynomial in the elements of $\psi_{i, j}$ of order 3 . Finally, the expression of $J(\mathbf{q}) \partial_{x_{i}}$ can also be expressed in terms of the displacement field. We first note that

$$
J(\mathbf{q}) \partial_{x_{i}}=J(\mathbf{q}) \frac{\partial q_{j}}{\partial x_{i}} \partial_{q_{j}}
$$

and $J(\mathbf{q}) \frac{\partial q_{j}}{\partial x_{i}}$ is given by determinant of the co-matrices. More precisely we have,

$$
J(\mathbf{q}) \frac{\partial q_{i^{\prime}}}{\partial x_{i}}=\frac{1}{2} \epsilon^{i j k} \epsilon^{i^{\prime} j^{\prime} k^{\prime}}\left(\delta_{j j^{\prime}}+\psi_{j, j^{\prime}}(\mathbf{q})\right)\left(\delta_{k k^{\prime}}+\psi_{k, k^{\prime}}(\mathbf{q})\right)
$$

which is a polynomial of order 2 in $\psi_{i, j}(\mathbf{q})$. The motion equation is therefore cubic in the displacement field and reads,

$$
\begin{aligned}
& \epsilon^{i j k} \epsilon^{i^{\prime} j^{\prime} k^{\prime}}\left(\delta_{j j^{\prime}}+\psi_{j, j^{\prime}}(\mathbf{q})\right)\left(\delta_{k k^{\prime}}+\psi_{k, k^{\prime}}(\mathbf{q})\right)\left[\psi_{i, i^{\prime}}^{\prime \prime}(\mathbf{q})+\left(\frac{3 \Omega_{m}}{2 f_{+}^{2}}-1\right) \psi_{i, i^{\prime}}^{\prime}(\mathbf{q})\right]= \\
& \frac{3 \Omega_{m}}{f_{+}^{2}}\left[\frac{1}{6} \epsilon^{i j k} \epsilon^{i^{\prime} j^{\prime} k^{\prime}}\left(\delta_{i i^{\prime}}+\psi_{i, i^{\prime}}(\mathbf{q})\right)\left(\delta_{j j^{\prime}}+\psi_{j, j^{\prime}}(\mathbf{q})\right)\left(\delta_{k k^{\prime}}+\psi_{k, k^{\prime}}(\mathbf{q})\right)-1\right] .
\end{aligned}
$$

It does not contain however all the required information: it is a scalar equation and as such it cannot allow to reconstruct the full displacement field. One should also take into account the absence of curl modes in the velocity field for the Eulerian coordinates,

$$
\nabla_{\mathbf{x}} \times \Psi^{\prime}(\mathbf{q})=0
$$

This constraint can easily be expressed using the form $(136)$. This is the form which is usually given in papers in the subject (see for instance Rampf and Buchert 2012). It is however possible to derive a simpler form which is a re-expression of the Kelvin's circulation theorem applied to our situation where we know from the Eulerian analysis that the curl term in the velocity field can be neglected (Rampf 2012; Frisch and Zheligovsky 2012). It leads to ${ }^{10}$

$$
\epsilon^{i j k} \psi_{l, i}^{\prime}\left(\delta_{l j}+\psi_{l, j}\right)=0 .
$$

Armed with the relations 137 and 139 one can then derive, order by order, the expression of the displacement field. The second order, third order and fourth order were subsequently derived in Bouchet et al. (1992), Bernardeau (1994b), Rampf and Buchert (2012), The second order expression revealed particularly useful for setting the initial conditions of numerical simulations as shown in Crocce et al. (2006)

\subsection{The Zel'dovich approximation and higher order solutions}

The much celebrated Zel'dovich approximation in cosmology (Zel'dovich 1970) is nothing but the Lagrangian mapping when the displacement field has been linearized. Note that in this case

\footnotetext{
${ }^{10} \mathrm{It}$ is actually not too difficult to derive this relation. The direct constraint, eqn 138 , leads to $\left(\delta_{k k^{\prime}}+\right.$ $\left.\psi_{k, k^{\prime}}\right) \epsilon^{i^{\prime} j^{\prime} k^{\prime}}\left(\delta_{j j^{\prime}}+\psi_{j, j^{\prime}}\right) \psi_{j, i^{\prime}}^{\prime}=0$ which is precisely satisfied if the relation 139 is valid provided the matrix $\left(\delta_{k k^{\prime}}+\psi_{k, k^{\prime}}\right)$ is regular.
} 
the divergence of the displacement field is solution of

$$
\psi^{\text {Zel. }_{i, i}{ }_{i, i}^{\prime \prime}}(\mathbf{q})+\left(\frac{3 \Omega_{m}}{2 f_{+}^{2}}-1\right) \psi^{\text {Zel. }{ }_{i, i}^{\prime}}(\mathbf{q})-\frac{3 \Omega_{m}}{2 f_{+}^{2}} \psi^{\text {Zel. }}{ }_{i, i}=0
$$

which is precisely the equation followed by the density contrast at linear oder. Moreover it is easy to see that at this order the displacement field is potential.

It is obviously possible to expand the solution to arbitrary orders. Note though that in general the displacement is not necessarily potential. The third order displacement field in particular is not potential anymore.

Form a diagrammatic representation point of view that makes the development of perturbation theory calculations much more intricate as potential and curl modes mix together. The diagrams are even more complicated since the equation of motion is third order in $\psi_{i, j}$ so that there are vertices with three incoming lines together with vertices with only two incoming lines.

\subsection{From displacement fields to power spectra}

Let us finish this section with a final ingredient, the calculation of power spectra (in Eulerian space) out of Lagrangian space calculations. The Eulerian Fourier modes are

$$
\delta(\mathbf{k})=\int \frac{\mathrm{d}^{3} \mathbf{x}}{(2 \pi)^{3 / 2}} e^{-\mathbf{i k} \cdot \mathbf{x}} \delta(\mathbf{x})
$$

or equivalently

$$
\delta(\mathbf{k})=\int \frac{\mathrm{d}^{3} \mathbf{x}}{(2 \pi)^{3 / 2}} e^{-\mathrm{ik} \cdot \mathbf{x}} \rho(\mathbf{x}),
$$

where the two expressions differ only by the $\mathbf{k}=0$ mode value. The expression of such a Fourier mode should then be expressed in terms of the Lagrangian variables. Taking advantage of the change of variable $\mathbf{x} \rightarrow \mathbf{q}$ with $\mathrm{d}^{3} \mathbf{q}=\rho(\mathbf{q}) \mathrm{d}^{3} \mathbf{x}$ one gets,

$$
\delta(\mathbf{k})=\int \frac{\mathrm{d}^{3} \mathbf{x}}{(2 \pi)^{3 / 2}} e^{-\mathrm{i} \mathbf{k} \cdot(\mathbf{q}+\Psi(\mathbf{q}))}(1-J(\mathbf{q}))
$$

in the first case and

$$
\delta(\mathbf{k})=\int \frac{\mathrm{d}^{3} \mathbf{x}}{(2 \pi)^{3 / 2}} e^{-\mathrm{ik} \cdot(\mathbf{q}+\Psi(\mathbf{q}))}
$$

in the second. The ensemble average of the product of two such wave modes can easily be done taking advantage of the statistical homogeneity of the universe. It leads to

$$
\left\langle\delta(\mathbf{k}) \delta\left(\mathbf{k}^{\prime}\right)\right\rangle=\delta_{\text {Dirac }}\left(\mathbf{k}+\mathbf{k}^{\prime}\right) \int \mathrm{d}^{3} \mathbf{q} e^{-\mathrm{ik} \cdot \mathbf{q}}\langle\exp [-\mathbf{i k} .(\Psi(\mathbf{q})-\Psi(0))]\rangle
$$

(when one uses the second formulation for the Fourier modes). For the Zel'dovich approximation the displacement field is Gaussian distributed, it is then possible to compute this expression using eqn (74).

In general though this is more cumbersome as the calculation of the ensemble average that appears in eqn 145 requires a full knowledge of the statistical properties of the displacement field correlators, not only the two-point correlation function.

These complications make Lagrangian Perturbation Theory very difficult to implement and much less developed than the Eulerian approaches. The Zel'dovich approximation though is a fantastic toy model to study the qualitative behavior of the cosmic web growth (see Bond 
Table 1: This table indicates what has been computed from direct PT calculation as a function of the order in multi-point correlators (lines) and the number of loops that have been taken into account in the calculations (columns).

\begin{tabular}{|c|c|c|c|c|c|}
\hline & tree & 1-loop & 2 loop & 3-loop & p-loop \\
\hline $\begin{array}{l}\text { 2-point } \\
\text { stats }\end{array}$ & OK & OK & OK & $\begin{array}{l}\text { partial inte- } \\
\text { grations }\end{array}$ & $\begin{array}{l}\text { partial re- } \\
\text { summation }\end{array}$ \\
\hline $\begin{array}{l}3 \text {-point } \\
\text { stats }\end{array}$ & OK & some terms & - & - & $\begin{array}{l}\text { partial re- } \\
\text { summation }\end{array}$ \\
\hline $\begin{array}{l}\text { 4-point } \\
\text { stats }\end{array}$ & OK & - & - & - & - \\
\hline $\begin{array}{l}\text { N-point } \\
\text { stats }\end{array}$ & $\begin{array}{l}\text { OK in spec } \\
\text { geometries }\end{array}$ & - & - & - & - \\
\hline
\end{tabular}

et al. 1996) and has often been used to give insights in the global statistical properties of the density fields. For instance, in case of the Zel'dovich approximation this is made possible because all the statistical properties of the deformation matrix, $\psi_{i j}$, in particular its eigenvalues are known (Doroshkevich 1970).

\section{Other observables}

It is time to move to other types of observables that can be built in continuous random fields. There are many of such observables and motivations for using one or the other are usually related to prejudices on the performances regarding the statistical errors or systematics. Let us mention a few of popular ones,

- peak statistics that focus on most prominent features of the field, see the paper of Bardeen et al. (1986) that set the stage for Gaussian fields and where the basics of such calculations are detailed;

- topological invariant and Minkowski functionals introduced in Gott (1988) and in Mecke et al. (1994) that aims at producing robust statistical indicators. How such observables are affected by weak deviations from Gaussianity was investigated in particular by Matsubara (1994) with the use of the Edgeworth expression. We will present this tool in the following;

- the study of extended topological invariants has been renewed with the later introduction of the notion of skeleton Colombi et al. (2000) and Gay et al. (2012)

- counts-in-cells statistics and void probabilities with many studies starting with White (1979) and a nice exhaustive study by Balian and Schaeffer (1989) we will exploit hereafter.

They all make intervene quantities that are beyond the two-point statistics. Whether they can be computed for the nonlinear density field depends then strongly on our ability to compute the global statistical properties of the density field beyond the linear regime. It turns out however that for most of those observables, calculations from first principles of related quantities are often challenging and in practice can be done for only a limited range of parameters and for weak departure from Gaussian statistics.

In Table 1, we set the stage on what have be computed from the motion equations. In the previous sections, we focused our interest in the first line corresponding to two-point correlators 
(either power spectra or two-point correlation functions.). The second and third lines correspond to the computation of bispectra and trispectra and although known results there are less detailed, such calculations can be addressed with the same tools.

In the next sections we would like to explore the first column. It corresponds to tree-order results obtained to correlators of arbitrary order. These results are obtained however for specific geometries and with methods that are totally different from those presented previously. But before we enter the heart of those calculations, we first present generic tools that are useful in this context.

\subsection{The inverse Laplace transform}

The purpose of this section is to compute the inverse Laplace transform expression in the context of cosmological density field. It is interesting to do the calculation starting with a discrete version of the probability distribution function (PDF), i.e. the calculation of the counts-in-cells probability, $P(N)$, that is the probability of having $N$ particles in one cell. We assume here that the discrete field is a Poisson realization of a continuous field with a global averaged number density of points given by $\bar{N}$, so that

$$
P(N)=\int \mathrm{d} \rho P_{\text {Poisson }}(N \mid \rho \bar{N}) \mathcal{P}(\rho)
$$

where $P_{\text {Poisson }}(N \mid \rho \bar{N})$ is the Poisson probability, i.e. the probability of having $N$ particles in a cell when the expected number density of particles is $\bar{N} \rho$,

$$
P_{\text {Poisson }}(N \mid \rho \bar{N})=\frac{(\bar{N} \rho)^{N}}{N !} \exp (-\bar{N} \rho)
$$

As a result one has

$$
P(N)=\int \mathrm{d} \rho \frac{(\bar{N} \rho)^{N}}{N !} \exp (-\bar{N} \rho) \mathcal{P}(\rho)
$$

It is then easy to show that

$$
\langle N\rangle=\bar{N}, \quad\langle N(N-1)\rangle=\bar{N}^{2}\left\langle\rho^{2}\right\rangle, \ldots
$$

and more generally that,

$$
\langle N \ldots(N-p+1)\rangle=\bar{N}^{p}\left\langle\rho^{p}\right\rangle .
$$

The quantities $\langle N \ldots(N-p+1)\rangle$ are called the factorial moments. They are directly proportional to the underlying density moments.

Let us then define the counts-in-cells generating function,

$$
\mathcal{D}(\mu)=\sum_{N} \mu^{N} P(N) .
$$

One can note that

$$
\mathcal{D}(1)=1, \frac{\partial}{\partial \mu} \mathcal{D}(1)=\langle N\rangle, \frac{\partial^{p}}{\partial \mu^{p}} \mathcal{D}(1)=\bar{N}^{p}\left\langle\rho^{p}\right\rangle
$$

and it finally implies that

$$
\mathcal{D}(\mu)=\mathcal{M}(\bar{N}(\mu-1))
$$

where $\mathcal{M}(\lambda)$ is the local density moment generating function defined in eqn $(72)$. Then we can use the residue theorem to relate the counts-in-cells statistics to the moment generating function,

$$
P(N)=\oint \frac{\mathrm{d} \mu}{2 \pi \mathrm{i}} \frac{1}{\mu^{(N+1)}} \mathcal{D}(\mu)=\frac{-1}{\bar{N}} \oint \frac{\mathrm{d} \mu}{2 \pi \mathrm{i}} e^{(-(N+1) \log [(1+\lambda) / \bar{N}])} \mathcal{M}(\lambda),
$$


where in the first integral the path line is a clockwise closed curve around the origin and in the second it is counterclockwise closed curve around $-\bar{N}$.

The continuous $\mathcal{P}(\rho)$ function is then obtained by taking the continuous limit $\bar{N} \rightarrow \infty$ while keeping $N / \bar{N}=\rho$ and $\lambda$ fixed, which leads to,

$$
\mathcal{P}(\rho) \mathrm{d} \rho=\mathrm{d} \rho \int_{-\mathrm{i} \infty}^{\mathrm{i} \infty} \frac{\mathrm{d} \lambda}{2 \pi \mathrm{i}} \exp (-\lambda \rho) \mathcal{M}(\lambda)
$$

where the integration path can be deformed along the imaginary axis. There ought to be singular points along the real axis in the positive domain otherwise the contour can be deformed away and the integral vanishes. This is the inverse Laplace transform. It gives the formal expression of the density PDF ${ }^{11}$ in terms of the moment generating function or equivalently of the cumulant generating function.

\subsection{The Edgeworth expansion}

The Edgeworth expansion has been developed in the context of small departure from a Gaussian statistics. It was originally developed by Longuet-Higgins in the context of sea wave statistics (Longuet-Higgins 1963). It was later introduced in the cosmological context by Juszkiewicz et al. (1995) and Bernardeau and Kofman (1995), There are different approaches that can be used to obtain this result. Here I focus on the method used in Bernardeau and Kofman (1995) which is based on the inverse Laplace transform.

As we are aiming at describing the density PDF is a regime where the variance is small it is natural to rely on the scaling relation that have been derived previously. In particular in the previous section we have seen that the cumulant generating function could be written

$$
\mathcal{C}(\lambda)=\frac{1}{\sigma^{2}} \varphi(y)
$$

with $y=\lambda \sigma^{2}$ and where $\varphi(y)$ remains finite in the small variance limit. The Edgeworth expansion is then obtained by a simple expansion of the moment generating function for terms that depart from a Gaussian distribution, i.e.

$$
\exp \left(\frac{1}{\sigma^{2}} \varphi(y)\right) \approx \exp \left(\frac{1}{2 \sigma^{2}} y^{2}\right)\left[1+\frac{1}{\sigma^{2}} S_{3} \frac{y^{3}}{3 !}+\frac{1}{\sigma^{2}} S_{4} \frac{y^{4}}{4 !}+\frac{1}{\sigma^{4}} S_{3}^{2} \frac{y^{6}}{2(3 !)^{2}}+\ldots\right] .
$$

It is to be noted that in this expansion $y$ will be eventually of the order of $\sigma$ so that the terms appearing in the expansion in the previous equation of the oder of $\sigma$, then of the order of $\sigma^{2}$, etc. The Edgeworh expansion is then obtained by a simple integral over $y$ which is then easy to do as $y$ is Gaussian distributed. We eventually get

$$
\begin{aligned}
\mathcal{P}(\rho)=\frac{1}{\left(2 \pi \sigma^{2}\right)^{1 / 2}} \exp \left(-\frac{\delta^{2}}{2 \sigma^{2}}\right) \quad & {\left[1+\sigma \frac{S_{3}}{6} H_{3}\left(\frac{\delta}{\sigma}\right)+\right.} \\
& \left.+\sigma^{2}\left(\frac{S_{4}}{24} H_{4}\left(\frac{\delta}{\sigma}\right)+\frac{S_{3}^{2}}{72} H_{4}\left(\frac{\delta}{\sigma}\right)\right)\right]
\end{aligned}
$$

where $H_{n}$ are the Hermite polynomials.

Such a construction can be extended to multiple variables (see for instance Amendola 1995) and is therefore of ubiquitous use when only a limited number of cumulants are known.

\footnotetext{
${ }^{11} \mathrm{~A}$ warning should be set here: there are cases where this inversion is not unique, that is, there exist families of distinct PDFs that exhibit the same cumulants to all orders. This is the case for the Lognormal distribution but this is normally not the case for the cosmological density PDF. See a recent paper by Carron and Neyrinck (2012) for explicit examples.
} 


\section{Cumulants in spherical cells}

The aim of this section is to present explicitly the cumulant generating functions that can be explicitly derived from the single flow Vlasov-Poisson system. They all concern variables that respect spherical symmetry: typically densities in concentric cells.

\subsection{Direct calculation of low order cumulants}

Let us start with a very simple and illustrative example, the third order cumulant of the local density contrast filtered at a given scale $R$. The shape of the filtering function is a priori arbitrary and we will denote it $\mathcal{W}(k R)$ when written in Fourier space. For functions that do not filter out the long-wave modes (such as Gaussian or top-hat filters), $\mathcal{W}(k R)$ is usually defined in such a way that $\mathcal{W}(0)=1$. Then the local filtered density ${ }^{12}$ field is,

$$
\delta_{R}=\int \frac{\mathrm{d}^{3}}{(2 \pi)^{3 / 2}} \delta_{R}(\mathbf{k}), \quad \delta_{R}(\mathbf{k})=\delta(\mathbf{k}) \mathcal{W}(k R)
$$

and this should obviously be true to all orders in perturbation theory,

$$
\delta_{R}^{(n)}=\int \Pi_{i}\left[\frac{\mathrm{d}^{3} \mathbf{k}_{i}}{(2 \pi)^{3 / 2}} \delta^{\operatorname{lin} .}\left(\mathbf{k}_{i}\right)\right] F_{n}\left(\mathbf{k}_{1}, \ldots, \mathbf{k}_{n}\right) \mathcal{W}\left(\left|\sum_{i} \mathbf{k}_{i}\right| R\right) .
$$

At linear order the only relevant quantity is the variance of the field, $\sigma_{R}$ given by,

$$
\sigma_{R}^{2}=\left\langle\delta_{R}^{2}\right\rangle=\int \frac{\mathrm{d}^{3} \mathbf{k}}{(2 \pi)^{3}} P^{\text {lin. }}(k) \mathcal{W}^{2}(k R) .
$$

Following the derivation of the scaling relation of Subsection 5.3 higher order cumulants will be given by a combination of factors taken at various orders. The practical difficulty in these calculations comes from the fact that the angular dependences between the wave modes are entangled in both the $F_{n}$ functions and the window function. For the third order cumulant the integral to compute is,

$$
\begin{aligned}
\left\langle\delta_{R}^{3}\right\rangle_{c}= & 6 \int \mathrm{d}^{3} \mathbf{k}_{1} \mathrm{~d}^{3} \mathbf{k}_{2} F_{2}\left(\mathbf{k}_{1}, \mathbf{k}_{2}\right) P^{\text {lin. }}\left(k_{1}\right) P^{\text {lin. }}\left(k_{2}\right) \\
& \times \mathcal{W}\left(k_{1} R\right) \mathcal{W}\left(k_{2} R\right) \mathcal{W}\left(\left|\mathbf{k}_{1}+\mathbf{k}_{2}\right| R\right) .
\end{aligned}
$$

Such calculation has been computed first in Juszkiewicz et al. (1993) for a Gaussian window function. It turns out however that the result takes a much simpler form for a filter which appears at first sight more complicated, the real space top-hat window function whose Fourier shape is given by 13

$$
\mathcal{W}(\mathbf{k} R)=\sqrt{\frac{3 \pi}{2}} \frac{J_{3 / 2}(k R)}{(k R)^{3 / 2}}
$$

in $3 \mathrm{D}$ where $J_{3 / 2}$ is the Bessel function of the first kind of index $3 / 2$. The calculation ${ }^{14}$ of 162 . makes indeed intervene only the second moment and its variation with the smoothing scale so that (Bernardeau 1994a),

$$
\frac{\left\langle\delta_{R}^{3}\right\rangle_{c}}{\left\langle\delta_{R}^{2}\right\rangle^{2}}=3 \nu_{2}+\frac{\mathrm{d} \log \sigma_{R}^{2}}{\mathrm{~d} \log R}
$$

\footnotetext{
${ }^{12}$ Although the calculations are presented here for the density modes, it is possible to do it in the velocity divergence field as well. It would follow exactly the same procedure.

${ }^{13}$ This is nothing but the Fourier transform of the characteristic function of a sphere of radius $R$.

${ }^{14} \mathrm{It}$ is based on the exploitation of summation theorem enjoyed by the Bessel functions, relation 8.530 of Gradshteyn and Ryzhik (1965)
} 
where $\nu_{2}$ is directly related to $F_{2}$ as its angular average,

$$
\nu_{2}=\int_{-1}^{1} \mathrm{~d} \mu F_{2}\left(\mathbf{k}_{1}, \mathbf{k}_{2}\right)
$$

( $\mu$ is the $\cos$ of the angle between $\mathbf{k}_{1}$ and $\mathbf{k}_{2}$ ). For an Einstein-de Sitter universe we have $3 \nu_{2}=34 / 7$. Such relation between the spherical collapse dynamics and tree-order cumulant can actually be generalized to all orders. This is this connexion that we will discuss in the rest of this section. First we need to explore a bit more the specificities of the spherical collapse solutions.

\subsection{The spherical collapse}

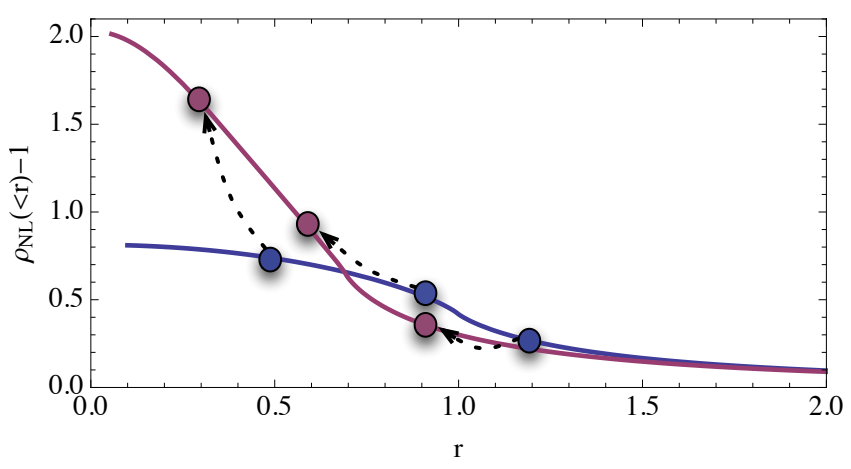

Figure 20: Example of evolution of a density profile with the spherical collapse. In blue we give the linearly evolved profile (linear growing mode), in red its nonlinear evolution: given a density contrast within a radius in the growing mode linear regime $\tau(<r)$ the subsequent shell size and density it encompasses are entirely determined by the spherical evolution - examples of such evolutions are given by the blue and red circles - which consequently determines the non-linear density profile

The spherical collapse does not only give the time it takes for a spherically symmetric perturbation to collapse, it also gives the explicit and exact solution of the nonlinear evolution of the density field before shell crossing for a wide class of initial fields, those with initial spherical perturbations. Moreover, the Gauss theorem ensures that the radius evolution of a shell in such a geometry is entirely determined by the total mass it contains. So let us consider a density contrast $\tau(<r)$ within the radius $r$. Let us call $R(\eta)$ the radius of that same shell during its nonlinear evolution and $\rho(<R, \eta)$ the total density it contains. At an arbitrarily early time the amount of matter encompassed within such a radius is simply $4 \pi / 3 r^{3} \bar{\rho}\left(\eta_{0}\right)$ and by matter conservation we have

$$
\rho(<R, \eta) R^{3}(\eta)=\bar{\rho}\left(\eta^{0}\right) r^{3} .
$$

The time evolution of $R$ can be solved in principle. It obeys the equation of motion

$$
\frac{\mathrm{d}^{2} R}{\mathrm{~d} t^{2}}=-\frac{G M(<R)}{R^{2}}
$$

which is nothing but the Friedman equation but for slightly different initial conditions. Matching the time variables in the two cases leads to an explicit form of the spherical collapse that relates the time dependent nonlinear density to the initial linear one when the latter is taken in the linear 
growing mode ${ }^{15}$. For an Einstein de Sitter background this equation is actually independent on time once the initial density contrast is expressed in terms of its linear evolution. Its explicit form depends on whether the initial perturbation, evolved linearly, $\tau$ is negative or positive. In the former case, we have,

$$
\rho(\tau)=\frac{9}{2} \frac{(\sinh \theta-\theta)^{2}}{(\cosh \theta-1)^{3}}, \quad \tau=-\frac{3}{5}\left[\frac{3}{4}(\sinh \theta-\theta)\right]^{2 / 3}
$$

and in the latter case,

$$
\rho(\tau)=\frac{9}{2} \frac{(\theta-\sin \theta)^{2}}{(1-\cos \theta)^{3}}, \quad \tau=\frac{3}{5}\left[\frac{3}{4}(\theta-\sin \theta)\right]^{2 / 3} .
$$

Another interesting peculiar case corresponds to the regime where the universe is almost empty $\left(\Omega_{m} \rightarrow 0\right.$ with $\left.\Omega_{\Lambda}=0\right)$ for which the spherical collapse solution takes a surprisingly simple form,

$$
\rho(\tau)=\frac{1}{(1-2 \tau / 3)^{3 / 2}}
$$

In the following we denote $\zeta(\tau)$ the functional form that relates the linear density contrast to the nonlinear density. It can formally be expanded in,

$$
\zeta(\tau)=\sum_{p} \nu_{p} \frac{\tau^{p}}{p !}
$$

The a priori time dependent $\nu_{p}$ parameters encode all the spherical collapse dynamics. And for the very same reason that the kernels $F_{n}$ and $G_{n}$ are almost independent on the background evolution, the function $\rho$, expressed as a function of the initial linear density contrast, is very weakly dependent on the cosmological parameters. The form 170 first proposed in Bernardeau $(1992)$, is actually very accurate in practice ${ }^{16}$.

What it implies is that for any initial spherical profile, that can always be characterized by the function $\tau\left(<r, \eta_{0}\right)$, the profile at time $\eta$ is given by

$$
\rho(<R, \eta)=\zeta\left[e^{\eta-\eta_{0}} \tau(<r)\right] \text {, with } \rho(<R, \eta) R^{3}=\bar{\rho}\left(\eta_{0}\right) r^{3} .
$$

Such a mapping is illustrated in Fig. 20

The explicit (or implicit) use of the spherical collapse solution is very common is cosmology and to a large extent to predict, at least roughly, the number density of formed halos and their correlation properties. There are many developments about this idea in textbooks (see also the review paper of Cooray and Sheth 2002) but the purpose of these notes is not to cover this field.

The spherical collapse solution can also be related to the full ensemble of the density cumulants. In the following we will make explicit use of the fact that this mapping provides an explicit non linear solution of the density field for spherically symmetric initial conditions.

\subsection{The tree order cumulant generating function as a Legendre trans- form of the initial moments}

We are interested here in the leading order expression of $\varphi\left(\left\{\lambda_{i}\right\}\right)$ for a finite number of concentric cells. The central result is that can be obtained is that it is given by the Legendre transform

\footnotetext{
${ }^{15}$ Setting the initial field in the growing mode is important otherwise one would need not only the initial density contrast but also the initial velocity gradient to predict the subsequent density evolution.

${ }^{16}$ Although it predicts a critical value for the density contrast, 1.5 , which is slightly below the value for an Einstein-de Sitter background, 1.69.
} 
of a function that can be built from the initial moments of the density taken in concentric cells. We refer to Valageas (2002) and Bernardeau et al. (2013) fo detailed demonstrations. In the following we simply explicit this result and present some of its applications.

So let us consider the joint cumulant distribution function of the cumulant of the densities in concentric cells,

$$
\varphi\left(\left\{\lambda_{k}\right\}\right)=\sum_{p_{i}=0}^{\infty}\left\langle\Pi_{i} \rho_{i}^{p_{i}}\right\rangle_{c} \frac{\Pi_{i} \lambda_{i}^{p_{i}}}{\Pi_{i} p_{i} !},
$$

where the densities $\rho_{i}$ are obtained as the density within the radius $R_{i}$ et time $\eta$,

$$
\rho_{i}(\eta)=\frac{1}{4 \pi R_{i}^{3} / 3} \int_{\left|\mathbf{x}-\mathbf{x}_{0}\right|<R_{i}} \mathrm{~d}^{3} \mathbf{x} \rho(\eta, \mathbf{x}) .
$$

To each such density $\rho_{i}$ and radius $R_{i}$ one can associate an initial density contrast $\tau_{i}$ computed at the smoothing radius $r_{i}=R_{i} \rho_{i}^{1 / 3}$ that follows the spherical collapse mapping,

$$
\rho_{i}(\eta)=\zeta\left(e^{\eta-\eta_{0}} \tau_{i}\right)
$$

Then what the Gauss theorem ensures is that the cumulant generating at leading order is entirely determined by the statistical properties of the variables $\tau_{i}$ at time $\eta_{0}$. So let us first define the cross-correlation of the initial density in cells of radii $r_{i}$

$$
\Sigma_{i j}=\left\langle\tau\left(<R_{i} \rho_{i}^{1 / 3}\right) \tau\left(<R_{j} \rho_{j}^{1 / 3}\right)\right\rangle .
$$

One can then define the function $\Psi$ as,

$$
\Psi\left(\left\{\rho_{i}\right\}\right)=\frac{1}{2} \sum_{i j} \Xi_{i j} \tau_{i} \tau_{j}
$$

seen as a function of the variable $\rho_{i}$ where $\Xi_{i j}$ is the inverse matrix of the cross-correlation matrix $\Sigma_{i j}$. The leading order expression of $\varphi$ is then given by the Legendre transform of the function $\Psi\left(\left\{\rho_{k}\right\}\right)$,

$$
\varphi\left(\left\{\lambda_{k}\right\}\right)=\sum_{i} \lambda_{i} \rho_{i}-\Psi\left(\left\{\rho_{k}\right\}\right)
$$

where the values of $\rho_{i}$ are determined by the stationary conditions,

$$
\lambda_{i}=\frac{\partial}{\partial \rho_{i}} \Psi\left(\left\{\rho_{k}\right\}\right) .
$$

This is this general expression that we will exploit in the following. Note that this relation is true for $\eta=\eta_{0}$ as we then have $\rho_{i}\left(\eta_{0}\right)=1+\tau_{i}$ and the fact that $\varphi\left(\left\{\lambda_{k}\right\}\right)$ and $\Psi\left(\left\{\rho_{k}\right\}\right)$ are Legendre transforms can easily be checked for a Gaussian field.

For $\eta>\eta_{0}$, the resulting cumulant generating function is no more quadratic. Higher order terms are induced due to the nonlinear $\tau_{i}-\rho_{i}$ mapping.

Naturally, re-expanding the function $\varphi\left(\left\{\lambda_{k}\right\}\right)$ with respect to $\lambda_{k}$ then gives back the individual cumulants. One can check for instance that the cubic term of the one-variable generating function following the prescription 178,179 gives back eqn 164 . This is not a very easy calculation can it can be implemented very efficiently in a formal calculator to any order in the expansion. It gives the explicit expression of any such cumulant or joint cumulant computed at tree order (explicit expressions have been given up to the tenth order cumulant!). 


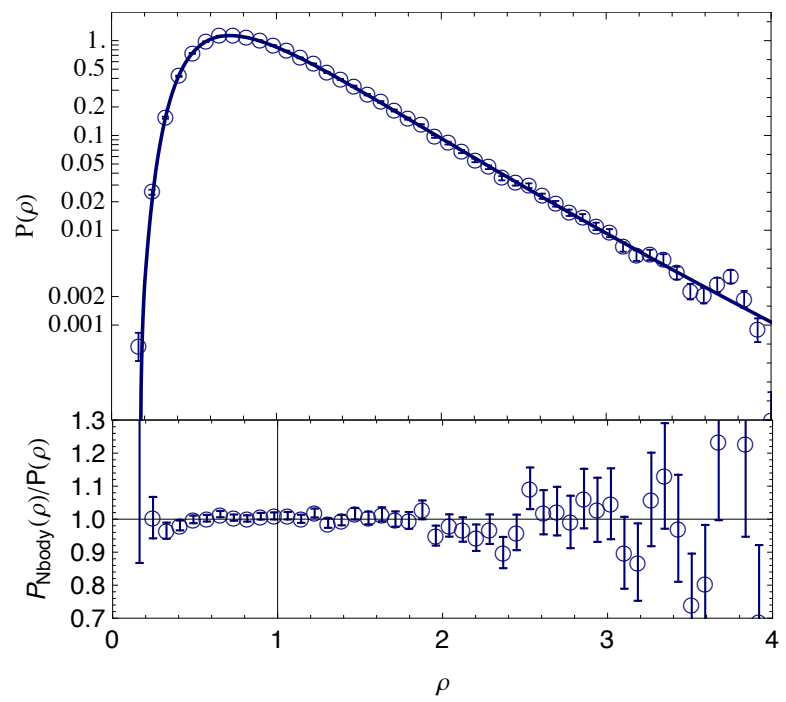

Figure 21: Comparison of the analytical prediction, blue solid line, with the numerical results. The bottom panel shows the residuals.

At this stage, what we have is a pure mathematical trick, an efficient way for obtaining full series of cumulant values sparing the task of wave mode integrations, as in eqn (162). This trick however works only at tree order. Comparisons of such predictions with simulations have been made in various papers and proved to be very accurate (Baugh et al. 1995. Bernardeau 1994a).

It is however possible to go one step further by assuming that the generating function are given by eqns 178 179) which corresponds to the vanishing variance limit, is actually a good approximation of the actual cumulant generating functions for finite but small values of the variance. It is possible to exploit this construction to built the corresponding PDF or joint PDF of the densities.

\section{Density PDFs and profiles with spherical cells}

\subsection{Reconstructing the density PDFs}

The reconstruction of the density PDFs are based on the inverse Laplace transforms, eqn (155), written in term of the cumulant generating function,

$$
\mathcal{P}(\hat{\rho})=\int_{-\mathrm{i} \infty}^{\mathrm{i} \infty} \frac{\mathrm{d} \lambda}{2 \pi \mathrm{i}} \exp [-\lambda \hat{\rho}+\varphi(\lambda)] .
$$

In the following we will denote with a ${ }^{\wedge}$ the random quantities for which we compute the statistical properties (to avoid confusion with those that appear in the Legendre transform). It is important to have in mind that the resulting one-point PDF depends strongly on the analytical structure of the cumulant generating functions (in this respect the key paper to explore in detail is Balian and Schaeffer (1989) which contains a description of all approximations and regimes one wishes to know about this transformation). The latter turns out to be determined to a large extent by the structure of the stationary condition $\sqrt{179}$. For the one-variable case, it appears that there is always a singular value on the real axis $\lambda_{c}>0$ beyond which there is no solution for $\lambda$ (this 
property appears on Fig. 22 when $\varphi(\lambda, \mu)$ is restricted to $\mu=0)$. It corresponds to a value $\rho=\rho_{c}$ at which location we have,

$$
\begin{aligned}
0 & =\Psi^{\prime \prime}\left[\rho_{c}\right] \\
\lambda_{c} & =\Psi^{\prime}\left[\rho_{c}\right]
\end{aligned}
$$

Note that at $\rho=\rho_{c}, \Psi$ is regular ${ }^{17}$ The function $\varphi(\lambda)$ can be expanded near this point and we typically get,

$$
\varphi(\lambda)=\varphi_{c}+\left(\lambda-\lambda_{c}\right) \rho_{c}+\frac{2}{3} \sqrt{\frac{2}{\pi_{3}}}\left(\lambda-\lambda_{c}\right)^{3 / 2}
$$

where $\pi_{i}=\partial^{i} \Psi / \partial \rho^{i}$. It is to be noted that the leading singular term scales like $\left(\lambda-\lambda_{c}\right)^{3 / 2}$. The coefficients $\pi_{i}$ are all related to the function $\Psi$ and are therefore model dependent 18

We can now re-construct the basic properties of the density PDF.

Let us focus to cases where the variance of the local density contrast is small. Then the inverse transform can in turn be obtained from a steepest descent method. Formally it leads to the conditions that should be met at the saddle point $\lambda_{s}$,

$$
\begin{aligned}
\frac{\partial}{\partial \lambda}[\lambda \hat{\rho}-\varphi(\lambda)] & =0 ; \\
\frac{\partial^{2}}{\partial \lambda^{2}}[\lambda \hat{\rho}-\varphi(\lambda)] & <0 .
\end{aligned}
$$

The first condition leads to $\rho\left(\lambda_{s}\right)=\hat{\rho}$ and the second to $\lambda_{s}<\lambda_{c}$. It simply means that this approximation can be used if $\hat{\rho}<\rho_{c}$. The resulting expression for the density PDF is easy to obtain and it leads to,

$$
\mathcal{P}(\hat{\rho})=\frac{1}{\sqrt{2 \pi}} \sqrt{\frac{\partial^{2} \Psi(\hat{\rho})}{\partial \rho^{2}}} \exp [-\Psi(\hat{\rho})] .
$$

It is valid as long as the expression that appears in the square root is positive which amounts to say that $\hat{\rho}<\rho_{c}$.

When this latter condition is not satisfied, the singular behavior of $\varphi$ dominates the integral in the complex plane. It eventually leads to

$$
\mathcal{P}(\hat{\rho}) \approx \frac{3 \Im\left(a_{\frac{3}{2}}\right)}{4 \sqrt{\pi}\left(\hat{\rho}-\rho_{c}\right)^{5 / 2}} \exp \left(\varphi_{c}-\lambda_{c} \hat{\rho}\right)
$$

where $a_{j}$ are the coefficient in front of $\left(\lambda-\lambda_{c}\right)^{j}$ in Eq. $\sqrt{183}$, e.g. $a_{3 / 2}=2 / 3 \sqrt{2 / \pi_{3}}$ and $\Im\left(a_{3 / 2}\right)$ is its imaginary part. It leads to exponential cut-off at large $\hat{\rho}$ as $\exp \left(\lambda_{c} \hat{\rho}\right)$. This property is actually robust and is preserved when one performs the inverse Laplace transform for finite values of the variance or even large value of the variance (see Balian and Schaeffer 1989). It also gives a direct transcription of why $\varphi(\lambda)$ becomes singular: for values of $\lambda$ that are larger than $\lambda_{c}$, $\int \mathrm{d} \hat{\rho} \mathcal{P}(\hat{\rho}) \exp (\lambda \hat{\rho})$ is not converging.

None of these forms however are accurate for the full range of density values and in general one has to rely on numerical integrations in the complex plane. Those can be performed accurately and quickly with a proper choice of the integration path in the complex plane (as described

\footnotetext{
${ }^{17}$ In particular this singular behavior in $\varphi(\lambda)$ we get is not related to any singularity in the spherical collapse.

${ }^{18}$ Note that in practice $\pi_{3}$ is negative and that only the non regular parts appearing in the expansion of $\exp (\varphi(\lambda))$ will contribute.
} 
for instance in Bernardeau 1992). The resulting shape for the PDF is shown on Fig. 21 and compared to results of numerical simulation. In this comparison we have $\sigma_{R}=0.47$ and a power spectrum index close to -1.47 . The predictions are shown to be accurate at percent level. They deteriorate when the variance gets comparable to unity.

Such calculations have been used for comparison with numerical simulation (as in Baugh et al. 1995; Bernardeau 1994a in the early papers and more recently in (Bernardeau, Pichon, and Codis 2013)), for projected density PDFs such as in Valageas (2000) or more recently in Munshi et al. (2013).

\subsection{Two-cell cumulant generating functions}

In this previous paragraph we applied the construction we found to the one-cell case. The whole procedure can actually by applied to a whole set of concentric cells. With two concentric densities, one can define the local slope - the density difference within two close radii - or more generally the profile - how the density depends on the filtering radius once a constraint has bet set at a fixed one. It is possible in particular to build marginal or constraint slope or profile PDF.

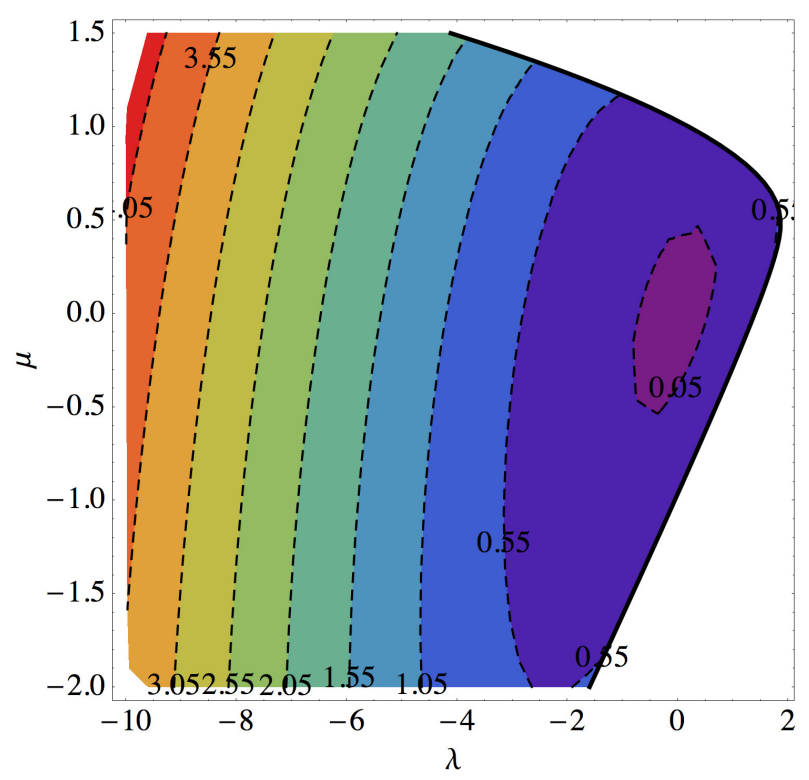

Figure 22: Contour plot of $\varphi(\lambda, \mu)-\lambda$, left with a finite radius difference $\Delta R / R=1 / 10$. The solid line shows the critical region. On the axis $\mu=0$ one recovers the analytical structure of the one-point generating function which is defined in a semi-infinite interval $\lambda<\lambda_{c}$.

All these constructions are based on the properties of the two-cell PDF or equivalently on the two-cell cumulant generating function. So we now turn to the analytical properties of $\varphi\left(\lambda_{1}, \lambda_{2}\right)$ for a finite radius difference. We represent this function, for a specific choice of radii but it does chance its expected properties, on Fig. 22. And to make the resulting plot easier to read we rather use,

$$
\lambda=\lambda_{1}+\lambda_{2}, \quad \mu=\frac{R_{2}-R_{1}}{R_{1}} \lambda_{1} .
$$


It is to be noted that, as for the $1 \mathrm{D}$ case, not all values of $\lambda_{1}$ and $\lambda_{2}$ (or equivalently $\lambda$ and $\mu$ ) are accessible. This is due to the fact that the $\rho_{i}-\lambda_{i}$ relation cannot always be inverted. This is signaled by the fact that the determinant of the transformation vanishes, i.e., $\operatorname{det}\left[\partial^{2} \Psi\left(\left\{\rho_{i}\right\}\right) / \partial \rho_{i} \partial \rho_{j}\right]=0$. This condition is met for finite values of both $\rho_{i}$ and $\lambda_{i}$. The resulting critical line is shown as a thick solid line on Figs. 22. Note that $\varphi\left(\lambda_{1}, \lambda_{2}\right)$ is also finite at this location. Within this line $\varphi$ is defined; beyond this line it is not.

\subsection{The expected density slope and profile}
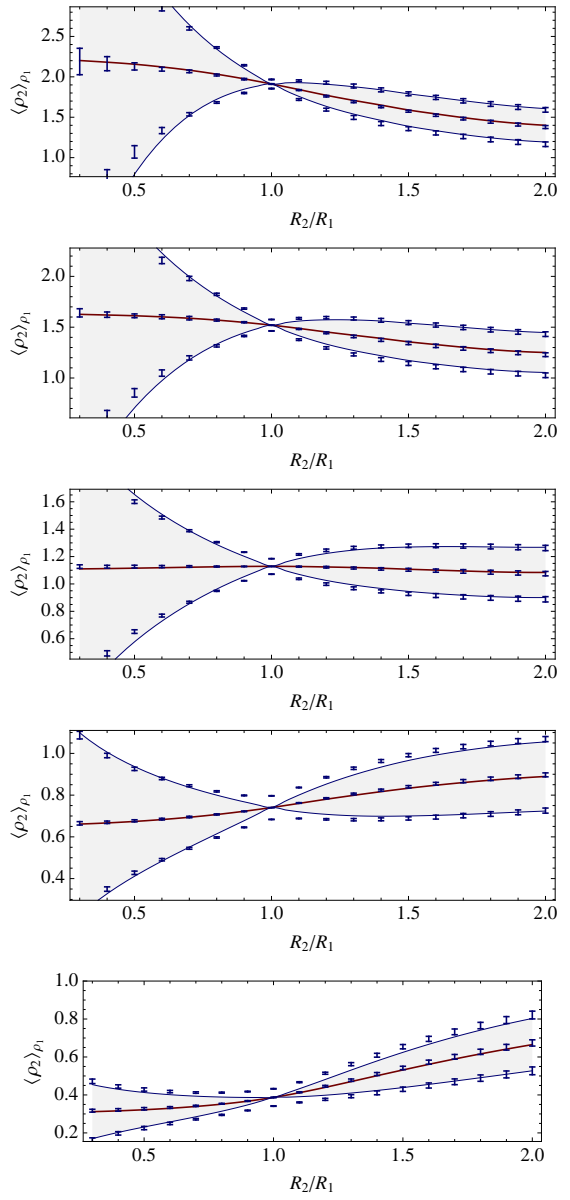

Figure 23: The conditional profile as a function of $R_{2}$ and for different choices of $\hat{\rho}\left(R_{1}\right)$ (to which $\hat{\rho}\left(R_{2}\right)$ is equal to at $\left.R_{2}=R_{1}\right)$ as predicted by theory and as measured in simulations. The solid lines are the theoretical predictions and the points with error bars are the measurements for both the expected value and its variance. The agreement is spectacular in particular for low density constraints. Taken from (Bernardeau et al. 2013).

As mentioned before, the general formalism allows in principle the computation of not only the one-point PDF but also of multi-variable density PDFs such as $\mathcal{P}\left(\hat{\rho}_{1}, \hat{\rho}_{2}\right)$. To illustrate one consequence of such calculations, one can also make a change of variable and consider the joint PDF of the density and the density slope. From the densities in two concentric cells, the latter 
can indeed be defined as 19

$$
\hat{s}\left(R_{1}, R_{2}\right)=\frac{R_{1}}{R_{1}-R_{2}}\left[\hat{\rho}_{1}-\hat{\rho}_{2}\right] .
$$

One of the (many) quantities that can explicitly be computed is the conditional slope that is the expected value of $\hat{s}$ given the value of $\hat{\rho}_{1}$,

$$
\langle\hat{s}\rangle_{\hat{\rho}_{1}}=-\frac{R}{\Delta R}+\frac{R}{\Delta R \mathcal{P}\left(\hat{\rho}_{1}\right)} \int \mathrm{d} \hat{\rho}_{2} \hat{\rho}_{2} \mathcal{P}\left(\hat{\rho}_{1}, \hat{\rho}_{2}\right) .
$$

Such a quantity can easily be expressed in terms of the joint cumulant generating function as,

$$
\int \mathrm{d} \hat{\rho}_{2} \hat{\rho}_{2} \mathcal{P}\left(\hat{\rho}_{1}, \hat{\rho}_{2}\right)=\left.\int_{-\mathrm{i} \infty+\epsilon}^{+\mathrm{i} \infty+\epsilon} \frac{\mathrm{d} \lambda_{1}}{2 \pi \mathrm{i}} \frac{\partial \varphi\left(\lambda_{1}, \lambda_{2}\right)}{\partial \lambda_{2}}\right|_{\lambda_{2}=0} \exp \left(-\lambda_{1} \hat{\rho}_{1}+\varphi\left(\lambda_{1}\right)\right)
$$

which can be obtained by explicit integration in the complex plane. Pursuing along the same line of calculations, the variance of $\hat{\rho}_{2}$ given $\hat{\rho}_{1}$ can be computed from the conditional value of $\hat{\rho}_{2}^{2}$. The latter is given by the second derivative of the moment generating function, therefore given by,

$$
\begin{array}{r}
\int \mathrm{d} \hat{\rho}_{2} \hat{\rho}_{2}^{2} \mathcal{P}\left(\hat{\rho}_{1}, \hat{\rho}_{2}\right)=\int_{-\mathrm{i} \infty+\epsilon}^{+\mathrm{i} \infty+\epsilon} \frac{\mathrm{d} \lambda_{1}}{2 \pi \mathrm{i}}\left[\left.\frac{\partial^{2} \varphi\left(\lambda_{1}, \lambda_{2}\right)}{\partial \lambda_{2}^{2}}\right|_{\lambda_{2}=0}\right. \\
\left.+\left(\left.\frac{\partial \varphi\left(\lambda_{1}, \lambda_{2}\right)}{\partial \lambda_{2}}\right|_{\lambda_{2}=0}\right)^{2}\right] \exp \left(-\lambda_{1} \hat{\rho}_{1}+\varphi\left(\lambda_{1}\right)\right) .
\end{array}
$$

Obviously such calculations can be pursued and can be used to predict the general statistical properties of the density profile as a function of the local density. Such predictions are shown on Fig. 23, where the expected value $\hat{\rho}_{2}\left(R_{2}\right)$ are given as a function of $R_{2}$ - we then build the constrained density profile - for various values of $\hat{\rho}_{1}\left(R_{1}\right)$, and compared the predictions with numerical simulations. When the variance is small the predictions proved to be very accurate, at percent level. The predictions are particularly appealing in the low density region as the fluctuations about the mean profile are expected to be limited 20 . With the advent of very large surveys, it opens the way to alternative ways of exploring the statistical properties of the surveys.

\section{Conclusion and perspectives}

The Vlasov-Poisson system in its single flow approximation offers a nice playground for theoretical physicists. It describes the development of gravitational instabilities in a pressureless medium before the first shell crossings and as such is an approximation to the true motion equations, the Vlasov-Poisson system. It is nonetheless a well defined mathematical system on its own and is the one on which perturbation theory calculations are all based. Progress has been made recently on the understanding of the interplay between dynamics, the structure of the cosmic field being expended to a given order with respect to the initial fields, and statistics, in particular for Gaussian initial conditions.

One of the major efforts that have been undertaken is the computation of next-to-leading order and next-to-next-to-leading order (the leading order being the linear theory) of the power

\footnotetext{
${ }^{19}$ In the limit of a vanishing smoothing radius difference, $\hat{s}$ will define the local density slope. In Bernardeau et al. (2013) we explicitly show that this limit is genuine and can be taken at the level of the generating function. ${ }^{20}$ The connexion with statistical indicators such as stacked voids (Lavaux and Wandelt 2012) is however yet to be done!
} 
cosmic field power spectra. In particular the mode coupling structure of the corresponding terms has been analyzed in detail as presented in these notes, both in the infrared and the ultra-violet domains. The UV behavior, i.e. the importance of the impact of the small-scale modes on the large scale modes is still to be assessed and analyzed in detail as it represents potentially a major limitation to systematic PT calculations. Remedies to this limitation may be brought by EFT approaches that aim at regularizing the UV effects.

These efforts should be viewed as part of a grander work program that encompasses the development of efficient analytical tools for predicting from first principles the behavior of the cosmic fields in the intermediate regime. It requires not only the prediction for the density power spectrum but also the determination of the theoretical uncertainties related to those predictions, the calculation of co-variances.

Readers should also keep in mind that, contrary to what these notes suggest, these calculations should also be applicable not only to the density field but also to quantities more closely related to actual observations. And if the application of those results to cosmic shear for instance is a priori quite straightforward, its application to galaxy catalogue is more challenging as one should take into account biasing, and the impact of redshift space distortions. The calculations should also be completed in order to accommodate more realistic cosmological fluids such as massive neutrino: ${ }^{21}$ (the existence of which we are sure of!). It should also be able to handle modified gravity model: 22

One could also think of applying similar calculations to related or more elaborate observables such as configuration space correlation functions and higher order correlation functions. In these notes we also showed that alternative approaches could be used to explore this very system. As it has been shown, for some specific variables, it is possible to compute the leading order generating function of the moments and to build the corresponding PDFs or joint PDFs. Similar construction may be doable for more elaborate quantities, alternative methods or new techniques may still be uncovered that would help filling Table 1.

\section{Acknowledgements}

I would like to thank to organizers of the school for giving me the opportunity to give this series of lecture and to write these notes. I hope they will be useful. I am particularly indebted to my recent collaborators Filippo Vernizzi, Román Scoccimarro, Martín Crocce, Atsushi Taruya, Takahiro Nishimichi, Christophe Pichon and Sandrine Codis for a large fraction of the recent developments presented here. This work is partially supported by the grant ANR-12-BS05-0002 of the French Agence Nationale de la Recherche.

\section{References}

Abarbanel, H. D. I. and C. Itzykson (1969). Relativistic eikonal expansion. Phys. Rev. Lett. 23, 53.

Amendola, L. (1995, April). Non-Gaussian Likelihood Function. ArXiv Astrophysics e-prints.

Balian, R. and R. Schaeffer (1989, August). Scale-invariant matter distribution in the universe. I - Counts in cells. Astr. \& Astrophys. 220, 1-29.

Bardeen, J. M., J. R. Bond, N. Kaiser, and A. S. Szalay (1986, May). The statistics of peaks of Gaussian random fields. Astrophys. J. 304, 15-61.

${ }^{21}$ See some attempts to incorporate the impact of massive neutrinos in perturbation theory calculations in Saito et al. 2008; Saito et al. 2009 Lesgourgues et al. 2009

${ }^{22}$ Some early analytical results have been obtained for instance in Koyama et al. 2009. Brax and Valageas 2012 Brax and Valageas 2013 Taruya et al. 2013. 
Baugh, C. M., E. Gaztanaga, and G. Efstathiou (1995, June). A comparison of the evolution of density fields in perturbation theory and numerical simulations - II. Counts-in-cells analysis. Mon. Not. R. Astr. Soc. 274, 1049-1070.

Bernardeau, F. (1992, June). The gravity-induced quasi-Gaussian correlation hierarchy. Astrophys. J. 392, 1-14.

Bernardeau, F. (1994a, November). The effects of smoothing on the statistical properties of large-scale cosmic fields. Astr. \& Astrophys. 291, 697-712.

Bernardeau, F. (1994b, May). The nonlinear evolution of rare events. Astrophys. J. 427, 51-71.

Bernardeau, F., S. Colombi, E. Gaztañaga, and R. Scoccimarro (2002, September). Large-scale structure of the Universe and cosmological perturbation theory. Phys. Rep. 367, 1-3.

Bernardeau, F., M. Crocce, and R. Scoccimarro (2012, June). Constructing regularized cosmic propagators. Phys. Rev. D 85(12), 123519.

Bernardeau, F., M. Crocce, and E. Sefusatti (2010, October). Multipoint propagators for non-Gaussian initial conditions. Phys. Rev. D 82(8), 083507.

Bernardeau, F. and L. Kofman (1995, April). Properties of the cosmological density distribution function. Astrophys. J. 443, 479-498.

Bernardeau, F., C. Pichon, and S. Codis (2013). Cosmological density profiles from perturbation theory.

Bernardeau, F., A. Taruya, and T. Nishimichi (2012, November). Cosmic propagators at twoloop order. ArXiv e-prints.

Bernardeau, F., N. van de Rijt, and F. Vernizzi (2012, March). Resummed propagators in multicomponent cosmic fluids with the eikonal approximation. Phys. Rev. D 85(6), 063509.

Bernardeau, F., N. Van de Rijt, and F. Vernizzi (2013, February). Power spectra in the eikonal approximation with adiabatic and nonadiabatic modes. Phys. Rev. D 87(4), 043530.

Binney, J., N. Dowrick, A. Fisher, and M. Newman (1992). The Theory of Critical Phenomena. Oxford University Press, 1992.

Binney, J. and S. Tremaine (1987). Galactic dynamics.

Blas, D., M. Garny, and T. Konstandin (2013a, September). Cosmological perturbation theory at three-loop order. ArXiv e-prints.

Blas, D., M. Garny, and T. Konstandin (2013b, April). On the non-linear scale of cosmological perturbation theory. ArXiv e-prints.

Blumenthal, G. R., S. M. Faber, J. R. Primack, and M. J. Rees (1984, October). Formation of galaxies and large-scale structure with cold dark matter. Nature 311, 517-525.

Bond, J. R. and G. Efstathiou (1984, October). Cosmic background radiation anisotropies in universes dominated by nonbaryonic dark matter. Astrophys. J. Letter 285, L45-L48.

Bond, J. R., L. Kofman, and D. Pogosyan (1996, April). How filaments of galaxies are woven into the cosmic web. Nature 380, 603-606.

Bouchet, F. R., S. Colombi, E. Hivon, and R. Juszkiewicz (1995, April). Perturbative Lagrangian approach to gravitational instability. Astr. \& Astrophys. 296, 575.

Bouchet, F. R., R. Juszkiewicz, S. Colombi, and R. Pellat (1992, July). Weakly nonlinear gravitational instability for arbitrary Omega. Astrophys. J. Letter 394, L5-L8. 
Brax, P. and P. Valageas (2012, September). Structure formation in modified gravity scenarios. Phys. Rev. D 86(6), 063512.

Brax, P. and P. Valageas (2013, July). Impact on the power spectrum of screening in modified gravity scenarios. Phys. Rev. D $88(2), 023527$.

Buchert, T. (1992, February). Lagrangian theory of gravitational instability of FriedmanLemaitre cosmologies and the 'Zel'dovich approximation'. Mon. Not. R. Astr. Soc. 254, $729-737$.

Buchert, T. (1993, January). Lagrangian perturbation theory - A key-model for large-scale structure. Astr. \& Astrophys. 267, L51-L54.

Buchert, T. (1994, April). Lagrangian Theory of Gravitational Instability of FriedmanLemaitre Cosmologies - a Generic Third-Order Model for Nonlinear Clustering. Mon. Not. R. Astr. Soc. 267, 811.

Carrasco, J. J. M., M. P. Hertzberg, and L. Senatore (2012, September). The effective field theory of cosmological large scale structures. Journal of High Energy Physics 9, 82.

Carron, J. and M. C. Neyrinck (2012, May). On the Inadequacy of N-point Correlation Functions to Describe Nonlinear Cosmological Fields: Explicit Examples and Connection to Simulations. Astrophys. J. 750, 28.

Colombi, S., D. Pogosyan, and T. Souradeep (2000, December). Tree Structure of a Percolating Universe. Physical Review Letters 85, 5515-5518.

Cooray, A. and R. Sheth (2002, December). Halo models of large scale structure. Phys. Rep. 372, 1-129.

Creminelli, P., J. Noreña, M. Simonović, and F. Vernizzi (2013, September). Single-Field Consistency Relations of Large Scale Structure. ArXiv e-prints.

Crocce, M., S. Pueblas, and R. Scoccimarro (2006, November). Transients from initial conditions in cosmological simulations. Mon. Not. R. Astr. Soc. 373, 369-381.

Crocce, M. and R. Scoccimarro (2006a, March). Memory of initial conditions in gravitational clustering. Phys. Rev. D 73(6), 063520-+.

Crocce, M. and R. Scoccimarro (2006b, March). Renormalized cosmological perturbation theory. Phys. Rev. D $73(6), 063519$.

Crocce, M., R. Scoccimarro, and F. Bernardeau (2012a, December). MPTBREEZE: a fast renormalized perturbative scheme. Mon. Not. R. Astr. Soc. 427, 2537-2551.

Crocce, M., R. Scoccimarro, and F. Bernardeau (2012b, July). MPTbreeze: A fast renormalized perturbative scheme. ArXiv e-prints.

Doroshkevich, A. G. (1970, October). Spatial structure of perturbations and origin of galactic rotation in fluctuation theory. Astrophysics 6, 320-330.

Frisch, U. and V. Zheligovsky (2012, December). A very smooth ride in a rough sea. ArXiv e-prints.

Fry, J. N. (1984, April). The Galaxy correlation hierarchy in perturbation theory. Astrophys. J. 279, 499-510.

Gay, C., C. Pichon, and D. Pogosyan (2012, January). Non-Gaussian statistics of critical sets in 2D and 3D: Peaks, voids, saddles, genus, and skeleton. Phys. Rev. D 85(2), 023011.

Goroff, M. H., B. Grinstein, S.-J. Rey, and M. B. Wise (1986, December). Coupling of modes of cosmological mass density fluctuations. Astrophys. J. 311, 6-14. 
Gott, III, J. R. (1988, November). Measuring the topology of large-scale structure in the universe. PASP/ 100, 1307-1313.

Gradshteyn, I. S. and I. M. Ryzhik (1965). Table of integrals, series, and products. New York: Academic Press.

Guth, A. H. and S.-Y. Pi (1985, October). Quantum mechanics of the scalar field in the new inflationary universe. Phys. Rev. D 32, 1899-1920.

$\mathrm{Hu}$, W. (1995, August). Wandering in the Background: A CMB Explorer. ArXiv Astrophysics e-prints.

Jain, B. and E. Bertschinger (1996, January). Self-similar Evolution of Gravitational Clustering: Is $\mathrm{N}=-1$ Special? Astrophys. J. 456, 43.

Juszkiewicz, R., F. R. Bouchet, and S. Colombi (1993, July). Skewness induced by gravity. Astrophys. J. Letter 412, L9-L12.

Juszkiewicz, R., D. H. Weinberg, P. Amsterdamski, M. Chodorowski, and F. Bouchet (1995, March). Weakly nonlinear Gaussian fluctuations and the edgeworth expansion. Astrophys. J. 442, 39-56.

Kehagias, A. and A. Riotto (2013, August). Symmetries and consistency relations in the large scale structure of the universe. Nuclear Physics B 873, 514-529.

Kiefer, C., D. Polarski, and A. A. Starobinsky (1998). Quantum-To Transition for Fluctuations in the Early Universe. International Journal of Modern Physics D 7, 455-462.

Koyama, K., A. Taruya, and T. Hiramatsu (2009, June). Nonlinear evolution of the matter power spectrum in modified theories of gravity. Phys. Rev. D $79(12), 123512$.

Lavaux, G. and B. D. Wandelt (2012, August). Precision Cosmography with Stacked Voids. Astrophys. J. 754, 109.

Lemaître, G. (1931, March). Expansion of the universe, The expanding universe. Mon. Not. R. Astr. Soc. 91, 490-501.

Lemaitre, G. (1934, January). Evolution of the Expanding Universe. Proceedings of the $N a$ tional Academy of Science 20, 12-17.

Lesgourgues, J., S. Matarrese, M. Pietroni, and A. Riotto (2009, June). Non-linear power spectrum including massive neutrinos: the time-RG flow approach. J. of Cosmology and Astr. Phys. 6, 17.

Liddle, A. R. and D. H. Lyth (2000, April). Cosmological Inflation and Large-Scale Structure. Cosmological Inflation and Large-Scale Structure, by Andrew R. Liddle and David H. Lyth, pp. 414. ISBN 052166022X. Cambridge, UK: Cambridge University Press, April 2000.

Linde, A. (2005, March). Particle Physics and Inflationary Cosmology. ArXiv High Energy Physics - Theory e-prints.

Longuet-Higgins, M. (1963). The effect of nonlinearities on statistical distributions in the theory of sea waves. J. Fluid. Mech. 17, 459-480.

Lyth, D. H. D. H. and A. A. Riotto (1999, June). Particle physics models of inflation and the cosmological density perturbation. Phys. Rep. 314, 1-2.

Makino, N., M. Sasaki, and Y. Suto (1992, July). Analytic approach to the perturbative expansion of nonlinear gravitational fluctuations in cosmological density and velocity fields. Phys. Rev. D 46, 585-602. 
Matarrese, S. and M. Pietroni (2007, June). Resumming cosmic perturbations. J. of Cosmology and Astr. Phys. 6, 26.

Matsubara, T. (1994, October). Analytic expression of the genus in a weakly non-Gaussian field induced by gravity. Astrophys. J. Letter 434, L43-L46.

Matsubara, T. (2008, March). Resumming cosmological perturbations via the Lagrangian picture: One-loop results in real space and in redshift space. Phys. Rev. D $77(6), 063530$.

McDonald, P. (2006, November). Clustering of dark matter tracers: Renormalizing the bias parameters. Phys. Rev. D $74(10), 103512$.

Mecke, K. R., T. Buchert, and H. Wagner (1994, August). Robust morphological measures for large-scale structure in the Universe. Astr. \& Astrophys. 288, 697-704.

Munshi, D., S. Joudaki, J. Smidt, P. Coles, and S. T. Kay (2013, February). Statistical properties of thermal Sunyaev-Zel'dovich maps. Mon. Not. R. Astr. Soc. 429, 1564-1584.

Peebles, P. J. E. (1980). The large-scale structure of the universe. Research supported by the National Science Foundation. Princeton, N.J., Princeton University Press, 1980. 435 p.

Peebles, P. J. E. (1982, December). Large-scale background temperature and mass fluctuations due to scale-invariant primeval perturbations. Astrophys. J. Letter 263, L1-L5.

Peloso, M. and M. Pietroni (2013, May). Galilean invariance and the consistency relation for the nonlinear squeezed bispectrum of large scale structure. J. of Cosmology and Astr. Phys. 5,31 .

Pichon, C. and F. Bernardeau (1999, March). Vorticity generation in large-scale structure caustics. Astr. \& Astrophys. 343, 663-681.

Pietroni, M. (2008, October). Flowing with time: a new approach to non-linear cosmological perturbations. J. of Cosmology and Astr. Phys. 10, 36.

Pietroni, M., G. Mangano, N. Saviano, and M. Viel (2012, January). Coarse-grained cosmological perturbation theory. J. of Cosmology and Astr. Phys. 1, 19.

Planck Collaboration, P. A. R. Ade, N. Aghanim, C. Armitage-Caplan, M. Arnaud, M. Ashdown, F. Atrio-Barandela, J. Aumont, C. Baccigalupi, A. J. Banday, and et al. (2013, March). Planck 2013 results. I. Overview of products and scientific results. ArXiv e-prints.

Pope, S. B. (2000, August). Turbulent Flows.

Pueblas, S. and R. Scoccimarro (2009, August). Generation of vorticity and velocity dispersion by orbit crossing. Phys. Rev. D 80(4), 043504-+.

Rampf, C. (2012, December). The recursion relation in Lagrangian perturbation theory. J. of Cosmology and Astr. Phys. 12, 4.

Rampf, C. and T. Buchert (2012, June). Lagrangian perturbations and the matter bispectrum I: fourth-order model for non-linear clustering. J. of Cosmology and Astr. Phys. 6, 21.

Saito, S., M. Takada, and A. Taruya (2008, May). Impact of Massive Neutrinos on the Nonlinear Matter Power Spectrum. Physical Review Letters 100(19), 191301.

Saito, S., M. Takada, and A. Taruya (2009, October). Nonlinear power spectrum in the presence of massive neutrinos: Perturbation theory approach, galaxy bias, and parameter forecasts. Phys. Rev. D 80(8), 083528.

Schmidt, F., D. Jeong, and V. Desjacques (2013, July). Peak-background split, renormalization, and galaxy clustering. Phys. Rev. D 88(2), 023515. 
Scoccimarro, R. (1998, October). Transients from initial conditions: a perturbative analysis. Mon. Not. R. Astr. Soc. 299, 1097-1118.

Scoccimarro, R. (2001). A New Angle on Gravitational Clustering. In J. N. Fry, J. R. Buchler, and H. Kandrup (Eds.), The Onset of Nonlinearity in Cosmology, Volume 927 of New York Academy Sciences Annals, pp. 13-+.

Scoccimarro, R. and J. Frieman (1996). Loop Corrections in Non-Linear Cosmological Perturbation Theory II. Two-point Statistics and Self-Similarity. Astrophys. J. 473, 620 .

Scoccimarro, R. and J. Frieman (1996, July). Loop Corrections in Nonlinear Cosmological Perturbation Theory. Astrophys. J. Suppl. Ser. 105, 37.

Seljak, U. and P. McDonald (2011, November). Distribution function approach to redshift space distortions. J. of Cosmology and Astr. Phys. 11, 39.

Somogyi, G. and R. E. Smith (2010, January). Cosmological perturbation theory for baryons and dark matter: One-loop corrections in the renormalized perturbation theory framework. Phys. Rev. D 81(2), 023524.

Stoyanov, J. (1987). Counterexamples in Probability, Probability and Statistics Series (Second edn. ed.). Wiley \& Sons.

Taruya, A., F. Bernardeau, T. Nishimichi, and S. Codis (2012, November). Direct and fast calculation of regularized cosmological power spectrum at two-loop order. Phys. Rev. D $86(10), 103528$.

Taruya, A. and T. Hiramatsu (2008, February). A Closure Theory for Nonlinear Evolution of Cosmological Power Spectra. Astrophys. J. 674, 617-635.

Taruya, A., K. Koyama, T. Hiramatsu, and A. Oka (2013, September). Beyond consistency test of gravity with redshift-space distortions at quasi-linear scales. ArXiv e-prints.

Tseliakhovich, D. and C. Hirata (2010, October). Relative velocity of dark matter and baryonic fluids and the formation of the first structures. Phys. Rev. D 82(8), 083520-+.

Valageas, P. (2000, April). Statistical properties of the convergence due to weak gravitational lensing by non-linear structures. Astr. \& Astrophys. 356, 771-787.

Valageas, P. (2002, February). Dynamics of gravitational clustering. II. Steepest-descent method for the quasi-linear regime. Astr. \& Astrophys. 382, 412-430.

Valageas, P. (2007, December). Using the Zeldovich dynamics to test expansion schemes. Astr. \& Astrophys. 476, 31-58.

White, S. D. M. (1979, January). The hierarchy of correlation functions and its relation to other measures of galaxy clustering. Mon. Not. R. Astr. Soc. 186, 145-154.

Zel'dovich, Y. B. (1970, April). Fragmentation of a homogeneous medium under the action of gravitation. Astrophysics 6, 164-174. 